\title{
HIGHLY COMPACT ACCELERATOR-DRIVEN SUBCRITICAL ASSEMBLY FOR MEDICAL AND INDUSTRIAL APPLICATIONS
}

\author{
Award Number: DE-FC07-03ID14502 \\ Final Summary Report \\ July 31, 2006 \\ University of California \\ Department of Nuclear Engineering \\ Berkeley, CA 94720 \\ Congressional District 9
}

\author{
Jasmina Vujic (PI) \\ (510) 643-9884 \\ vujic@nuc.berkeley.edu \\ William Kastenberg (Co-PI) \\ (510) 643-0574 \\ kastenbe@nuc.berkeley.edu
}

Ehud Greenspan (Co-PI)

(510) 643-9983

gehud@nuc.berkeley.edu

Ka-Ngo Leung (Co-PI)

(510) 486-7918

knleung@lbl.gov 


\section{TABLE OF CONTENTS}

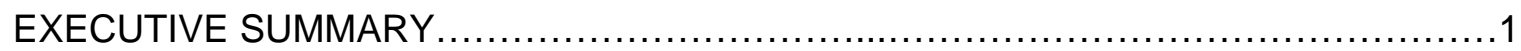

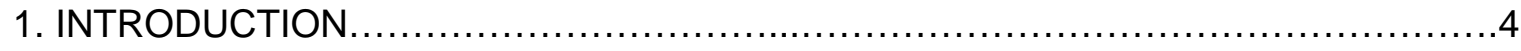

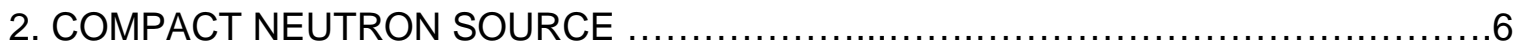

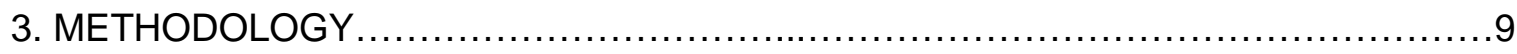

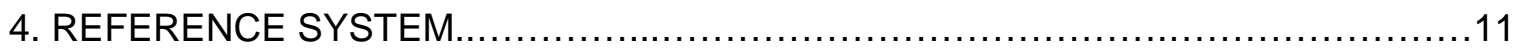

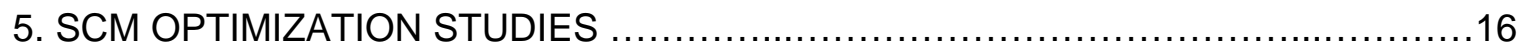

5.1 Cylindrical Sub-Critical Neutron Multiplier (SCM) optimization ....................16

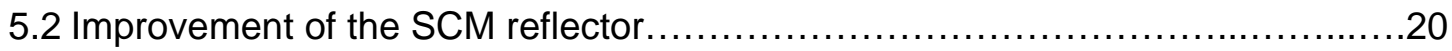

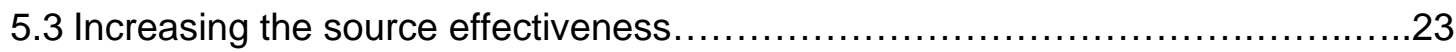

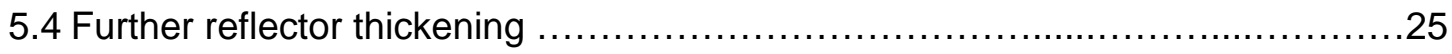

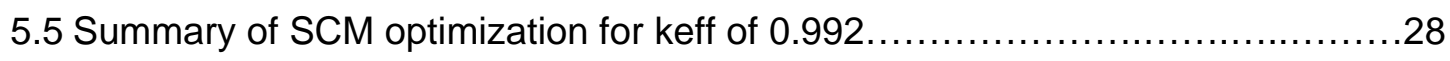

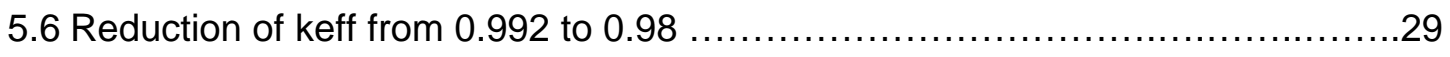

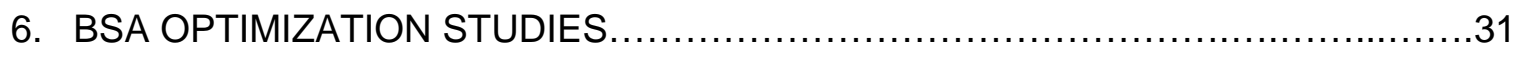

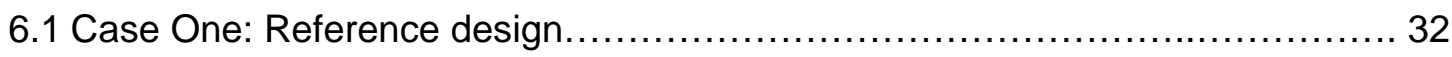

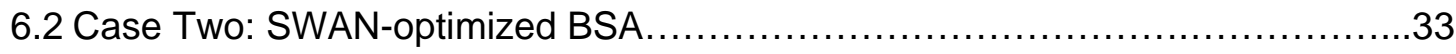

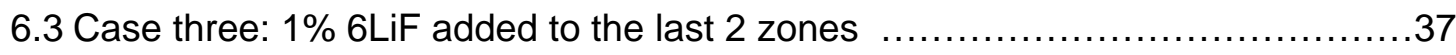

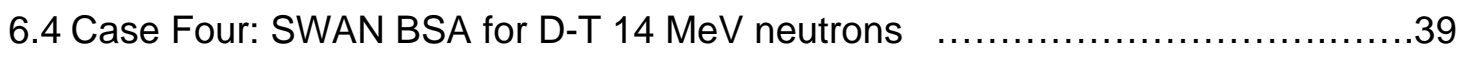

6.5 Case Five: Like Case 2 using Fluental in the last two zones ......................42

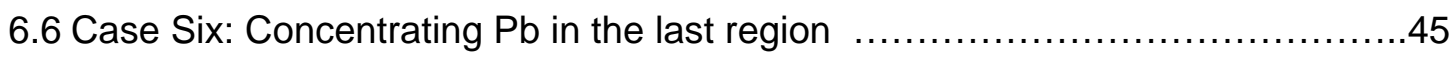

6.7 Case Seven: Lead is eliminated from the last three layers $\ldots \ldots \ldots \ldots \ldots \ldots \ldots \ldots \ldots .48$

6.8 Case Eight: Fluental added to the last part of the BSA ….......................51

6.9 Case Nine: Like Case One with Pb layer added at the exit of the BSA ...........53

6.10 Case Ten: Like Case 9 but with a Pb collar added ….......................57

6.11 Case Eleven: Like Case Eight with a lead "collar" ............................59 
6.12 Case Twelve: Like Case One but 6LiF extended up to the SCM ..............6 62

6.13 Case Thirteen: BeO instead of lead reflector for the BSA $\ldots \ldots \ldots \ldots \ldots \ldots \ldots . \ldots 6$

6.14 Case Fourteen: BeO instead of Alumina reflector for the BSA; Part II ......... 67

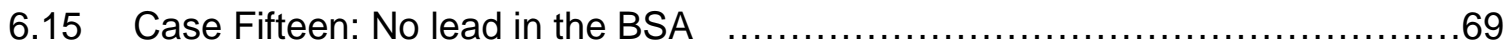

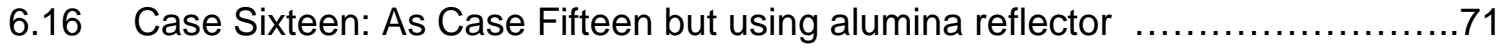

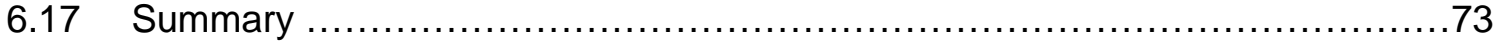

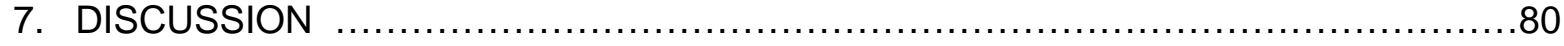

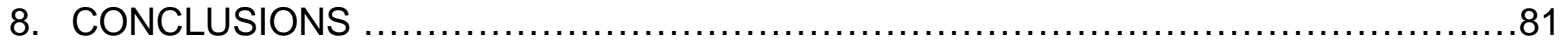

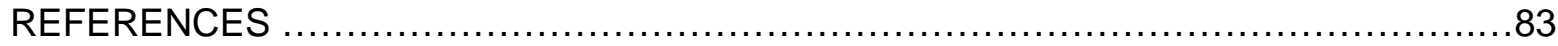

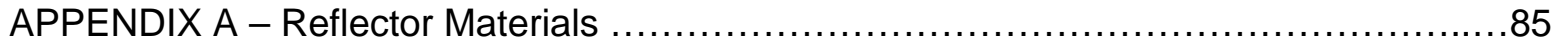




\section{EXECUTIVE SUMMARY}

A novel, highly compact, fusion neutron source (CNS) based on a coaxial electrostatic accelerator is under development at the Lawrence Berkeley National Laboratory. This source is designed to generate up to $\sim 10^{12} \mathrm{D}-\mathrm{D} \mathrm{n} / \mathrm{s}$. This source intensity is an order of magnitude too small for Boron Neutron Capture Therapy (BNCT) applications. The objective of this project is to assess the feasibility of using a small, safe and inexpensive subcritical fission assembly to multiply the fusion neutrons by a factor of $\geq 30$. The overall design objective is to get a treatment time for deep seated rain tumors that does not significantly increase beyond one hour when the effective multiplication factor of the SCM is $\mathrm{k}_{\text {eff }}=0.98$.

There are two major parts to this study: the optimization of the Sub-Critical Multiplier (SCM) and the optimization of the Beam Shaping Assembly (BSA), including the reflector for both subsystems. The SCM optimization objective is to maximize the current of neutrons that leak out from the SCM in the direction of the patient, without exceeding the maximum permissible $\mathrm{k}_{\text {eff. }}$. Minimizing the required uranium inventory is another objective. SCM design variables considered include the uranium enrichment level in the range not exceeding $20 \%{ }^{235} \mathrm{U}$ (for proliferation concerns), SCM geometry and dimensions, fuel thickness and moderator thickness.

The objective of the BSA optimization is to maximize the tumor dose rate using the optimal SCM while maintaining a tumor-to-normal tissue dose ratio of at least 20 to 12.5 (corresponding to the tumor control dose and to the healthy tissue dose limit). The BSA design variables include its shape, dimensions and composition. The reflector optimization is, in fact, an integral part of the SCM optimization and of the BSA optimization. The reflector design variables are composition and thickness.

The study concludes that it is not quite feasible to achieve the project objective. Nevertheless, it appears feasible to develop a practical BNCT facility based on the optimal system identified in this study by either

o Irradiating the patient in 3 to 4 one-hour sessions

o Irradiating the patient using 3 or 4 beams simultaneously

o Enhancing the capacity of the CNS to $\sim 5 \times 10^{12} \mathrm{D}-\mathrm{D} \mathrm{n} / \mathrm{s}$ 
o Increasing the permissible SCM maximum $\mathrm{k}_{\mathrm{eff}}$ to 0.995.

The optimal design of a SCM is made of $20 \%$ enriched uranium metal arranged as follows: two cylindrical fuel shells surrounds the CNS coaxially and four circular fuel elements are locate inbetween the CNS and the BSA. The coaxial cylinders are $21 \mathrm{~cm}$ long - as is the CNS, and 0.06 $\mathrm{cm}$ thick and are clad with $0.05 \mathrm{~cm}$ thick aluminum on both sides. Water fills the $0.84 \mathrm{~cm}$ gap between them. The innermost cylinder has a radius of $13.5 \mathrm{~cm}$. The disc-shaped fuel plates are, similarly, $0.06 \mathrm{~cm}$ thick, clad with $0.05 \mathrm{~cm}$ thick aluminum and have $0.84 \mathrm{~cm}$ thick water gaps in-between. The entire SCM is encased in aluminum structure that provides a physical barrier between the CSN and the SCM. A $0.84 \mathrm{~cm}$ thick water layer separates the outermost fuel element and the encasement. The total amount of enriched uranium in the SCM is $8.5 \mathrm{~kg}$ and its cost (not including fabrication) is estimated to be less than $\$ 60,000$. The SCM power level is estimated at $400 \mathrm{~W}$ when driven by a $10^{12} \mathrm{D}-\mathrm{D} \mathrm{n} / \mathrm{s}$ neutron source. This translates into consumption of only about $0.5 \%$ of the initially loaded ${ }^{235} \mathrm{U}$ atoms during 50 years of continuous operation. It implies that the SCM could operate continuously for the entire lifetime of the machine without refueling. Cooling the SCM does not pose a challenge; it may be accomplished passively; i.e., without resorting to forced circulation. For comparison, the power required for generating $10^{12} \mathrm{D}-\mathrm{D}$ neutron per second is approximately $400 \mathrm{KW}$.

Two optimal BSA designs were identified; one for maximizing the dose rate to a deep seated tumor and the other for maximizing the total dose that can be delivered to a deep seated tumor. The former offers the minimum treatment time whereas the latter offers a larger lethality range. The former features a harder neutron spectrum and relatively high neutron dose component to the skin while in the latter the neutron, gamma-ray and boron dose components in the skin are comparable. The maximum dose rate that can be delivered by the former is $10.1 \mathrm{~Gy} /$ hour and the maximum dose that can be delivered by the latter is $51.8 \mathrm{~Gy}$.

The BSA design that maximizes the dose rate is $51.1 \mathrm{~cm}$ long, with a truncated conical shape with the large base towards the SCM and the small base towards the patient. The angle of the cone is 14.9 degrees, the large base towards the SCM has a radius of $23.3 \mathrm{~cm}$ while the small base towards the patient has a radius of $9.74 \mathrm{~cm}$. The BSA is made of the following segments, 
starting from the SCM with the thickness indicated in $\mathrm{cm}: 3.5 \mathrm{~cm}$ lead, $6 \mathrm{~cm}{ }^{7} \mathrm{LiF}, 1.5 \mathrm{~cm}$ lead, $17 \mathrm{~cm}^{7} \mathrm{LiF}, 4 \mathrm{~cm}$ fluental, $1 \mathrm{~cm}$ lead, $8 \mathrm{~cm}$ fluental, $1 \mathrm{~cm}$ lead, $8 \mathrm{~cm}$ fluental, $0.9 \mathrm{~cm}$ lead and 0.1 $\mathrm{cm}{ }^{6} \mathrm{LiF}$. The cone is surrounded on the side by a $1 \mathrm{~mm}$ thick ${ }^{6} \mathrm{LiF}$ in the last $19 \mathrm{~cm}$ towards the patient. The reflector is made of lead, and fills the space between the side of the BSA cone and a coaxial cylinder $77 \mathrm{~cm}$ in radius. The sides of the collimator are shielded by a lead collar $2.1 \mathrm{~cm}$ thick, continuing the conical shape of the BSA.

The BSA design that maximizes the total dose is $53.1 \mathrm{~cm}$ long, with a truncated conical shape with the large base towards the SCM and the small base towards the patient. The angle of the cone is 14.9 degrees, the large base towards the SCM has a radius of $23.3 \mathrm{~cm}$ while the small base towards the patient has a radius of $9.18 \mathrm{~cm}$. The BSA is made of the following segments, starting from the SCM with the thickness indicated in $\mathrm{cm}: 3.5 \mathrm{~cm}$ lead, $27.4 \mathrm{~cm}{ }^{7} \mathrm{LiF}, 20 \mathrm{~cm}$ fluental, $0.1 \mathrm{~cm}{ }^{6} \mathrm{LiF}$ and $2.1 \mathrm{~cm}$ lead. The cone is surrounded on the side by a $1 \mathrm{~mm}$ thick ${ }^{6} \mathrm{LiF}$ in the last $21 \mathrm{~cm}$ towards the patient. The reflector is made of $\mathrm{Al}_{2} \mathrm{O}_{3}$, filling the space between the side of the BSA cone and a coaxial cylinder $35 \mathrm{~cm}$ in radius; lead surrounds the alumina reflector with a coaxial cylinder $77 \mathrm{~cm}$ in external radius. The sides of the collimator are shielded by a lead collar $2.1 \mathrm{~cm}$ thick, continuing the conical shape of the BSA.

It is recommended to assess the feasibility of designing an ever-safe SCM featuring a $\mathrm{k}_{\mathrm{eff}}$ of 0.995 . 


\section{INTRODUCTION}

Boron Neutron Capture therapy (BNCT) is being developed for, primarily, treatment for "glioblatoma multiforme", otherwise un-treatable form of malignant brain tumor, which afflicts about 15,000 new cases per year in the US alone. This type of cancer is characterized by lack of localization, so it is almost impossible to treat surgically.

BNCT is a two-steps process in which first a ${ }^{10} \mathrm{~B}$-carrying tumor-selective compound is injected to the patient. After that the patient is exposed to a beam of neutrons. Low energy neutrons absorbed by the ${ }^{10} \mathrm{~B}$ generate high energy ${ }^{7} \mathrm{Li}$ and ${ }^{4} \mathrm{He}$ reaction products via the following reactions:

$$
\begin{array}{lll}
{ }^{10} \mathrm{~B}+\mathrm{n} \rightarrow{ }^{7} \mathrm{Li}+\alpha+\gamma & (\mathrm{Q}=0.48 \mathrm{MeV}) & \text { in } 93 \% \text { of the cases; } \\
{ }^{10} \mathrm{~B}+\mathrm{n} \rightarrow{ }^{7} \mathrm{Li}+\alpha & (\mathrm{Q}=2.79 \mathrm{MeV}) & \text { in } 7 \% \text { of the cases. }
\end{array}
$$

These reaction products ionize and kill the cells in close proximity to their point of birth that are mostly cancerous cells. Additional neutron induced energy deposition reactions that take place in the brain are

$$
{ }^{14} \mathrm{~N}(\mathrm{n}, \mathrm{p}){ }^{14} \mathrm{C} \quad\left(\mathrm{E}_{\mathrm{p}}=0.626 \mathrm{MeV}\right),{ }^{1} \mathrm{H}(\mathrm{n}, \gamma){ }^{2} \mathrm{H} \quad\left(\mathrm{E}_{\gamma}=2.2 \mathrm{MeV}\right),
$$

as well as elastic scattering of fast neutrons with, primarily, the hydrogen nuclei. Figure 1 shows the cross sections for the three important neutron absorption reactions.

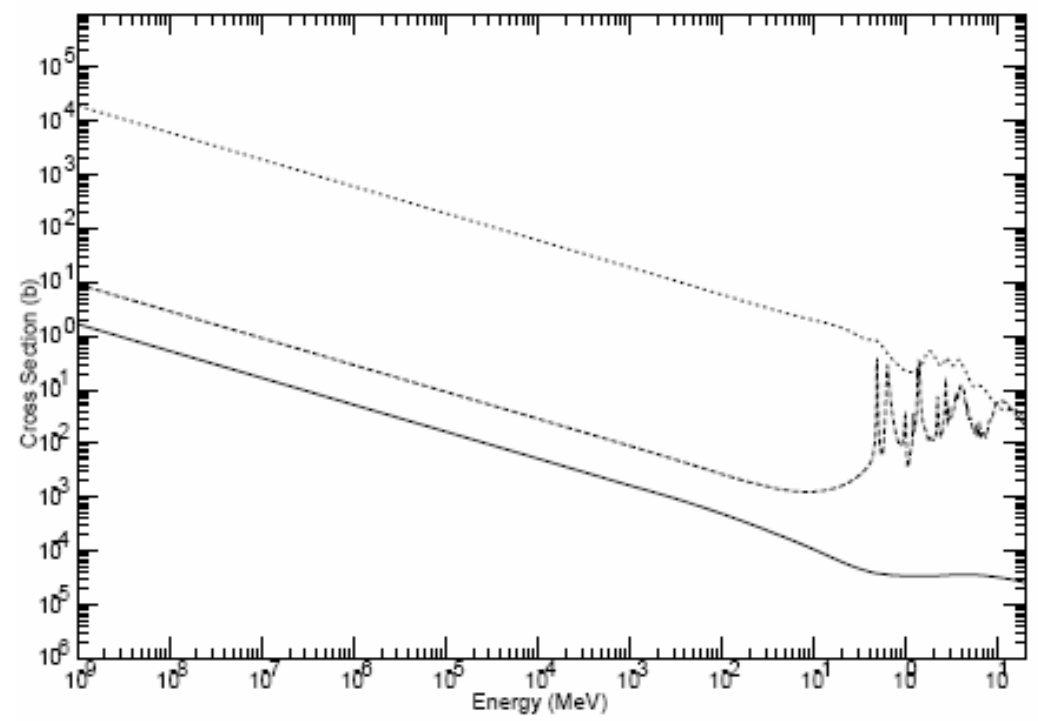

Figure 1: Cross sections of interest for BNCT. From top to bottom: ${ }^{10} \mathrm{~B}(\mathrm{n}, \alpha),{ }^{14} \mathrm{~N}(\mathrm{n}, \mathrm{p}),{ }^{1} \mathrm{H}(\mathrm{n}, \gamma)$ 
All the clinical trials of BNCT used neutron beams from nuclear reactors. Nuclear reactors are quite expensive and difficult to install and operate in hospitals. Accelerator neutron sources were also considered for BNCT but the presently available accelerator neutron sources that can provide the required neutron beam intensity are also quite large and expensive.

A novel, highly compact, fusion neutron source (CNS) based on a coaxial electrostatic accelerator is under development at the Lawrence Berkeley National Laboratory. This source is designed to generate up to $\sim 10^{12} \mathrm{D}-\mathrm{D} \mathrm{n} / \mathrm{s}$. This source intensity is an order of magnitude too small for Boron Neutron Capture Therapy (BNCT) applications. The primary objective of this project is to assess the feasibility of using a small, safe and inexpensive subcritical fission assembly to multiply the fusion neutrons by a factor of $\geq 30$ thus making a highly compact neutron source for BNCT and other medical and industrial applications.

Possible advantages of using a Sub-Critical neutron Multiplier (SCM) include the following: (a) Earlier commercialization of the CNS for BNCT, isotope generation and other possible medical and industrial applications. (b) Making it possible to attain the needed neutron source intensity using a D-D CNS. Without a SCM it is not likely that a D-D CNS could be developed to generate $\sim 10^{14} \mathrm{n} / \mathrm{s}$. The advantage of a D-D over a D-T neutron source is lack of need for tritium. Tritium is very expensive and is a health hazard. Designing the facility to confine tritium is expensive. (c) Reducing the tritium consumption rate of D-T CNS by a factor of at least 30. (c) Reducing the power needed for operating a CNS without a SCM by a factor of at least 30 . The $\mathrm{SCM}$ we have in mind is small and have low power - approximately $0.5 \mathrm{~kW}$ relative to $200 \mathrm{~kW}$ that will be deposited by the ion beam on the target of the D-D CNS described above. At that low power level it is possible to design the SCM to be passively super-safe. It can be designed in such a way that will not enable it to become critical under any circumstances. Passive cooling will ensure that the fuel will maintain its integrity and fission products will not be released. It will use a relatively small amount of low enriched uranium so it will not be of proliferation concern.

If feasible, the compact (possibly mobile) accelerator-driven system will provide an intense enough neutron source for medical applications in hospitals - for BNCT and other neutron therapy applications, for short-lived isotope production, as well as for research and application in material science, biology and other areas. The use of the subcritical multiplier will reduce, by an order of magnitude, the amount of electricity and tritium consumed by the CNS. 
The primary feasibility issue addressed in this project is the feasibility of designing a SCM using uranium enriched to up to $20 \%{ }^{235} \mathrm{U}$ that will be able to multiply the neutron current for patient treatment by at least a factor of 30 and, yet, be safe and economical.

An attempt at designing a SCM and BSA undertaken during the second year of the project did not yield satisfactory results. Hence, during the third year of the project we embarked upon a careful optimization studies attempting to reach the design goal for neutron beam intensity amplification. The optimization effort focused on, primarily, two components of the BNCT facility - the SCM and the BSA. Reflector/shield optimization was undertaken as well.

Following a description, in Section 2, of the compact neutron source considered in this project, we describe the starting point for the intensive optimization effort (Section 3), the methodology used for the optimization (Section 4), the optimization of the SCM (Section 5), the optimization of the BSA (Section 6) and close with a discussion (Section 7) and conclusions of this project (Section 8).

\section{COMPACT NEUTRON SOURCE}

A novel, highly compact, fusion neutron source (CNS) based on a coaxial electrostatic accelerator is under development at the Lawrence Berkeley National Laboratory. This source is designed to generate up to $\sim 10^{14} \mathrm{D}-\mathrm{T}$ neutrons $/ \mathrm{sec}$ or $\sim 10^{12} \mathrm{D}-\mathrm{D} \mathrm{n} / \mathrm{s}$.

Figure 2 shows the geometry and structure of the highly compact fusion neutron sources (CNS) under development $[1,2,3]$ at the Lawrence Berkeley National Laboratory (LBNL) with the participation of UC Berkeley Nuclear Engineering students. They are based on a newly developed RF-driven multicusp ion source that is simple to operate and has long lifetime. The cylindrical source consists of a radio frequency driven D-D or D-T plasma. A $2 \mathrm{MHz}$ or a 13.56 $\mathrm{MHz}$ radio frequency (RF) discharge produces the deuterium and/or tritium ions at the center of the cylindrical device. The RF-discharge yields a high-density plasma with a high fraction of mono-atomic ion species $\left(\mathrm{D}^{+}\right.$for $\mathrm{D}-\mathrm{D}$ and $50 \% \mathrm{D}^{+}+50 \% \mathrm{~T}^{+}$for $\left.\mathrm{D}-\mathrm{T}\right)$. It enables attaining high neutron yield with low gas pressures (few mTorr). A vacuum contained within a quartz vacuum chamber is used for high voltage insulation around the target cylinder. The vacuum line, RF power, cooling water, and pulsed high voltage are brought in through a vacuum feed-through at one end of the cylindrical assembly. 
The ions are accelerated through the extraction grid to an energy of $100 \mathrm{keV}$ or higher. The cylindrical geometry of the source is advantageous for holding high voltage due to the uniform distribution of the electric field equi-potential lines between the electrode and target cylinders. There are no sharp corners or edges that can cause field enhancements that may lead to electron emission and voltage breakdown. This geometry also simplifies the beam optics such that the beam power is evenly distributed over the cylindrical target, simplifying heat removal. Because this target is wrapped around the source of deuterium ions, there is a large target area in a small space compared to an accelerator with flat plate target. This geometry also leads to a very high current limit as a result of the large beam extraction area.
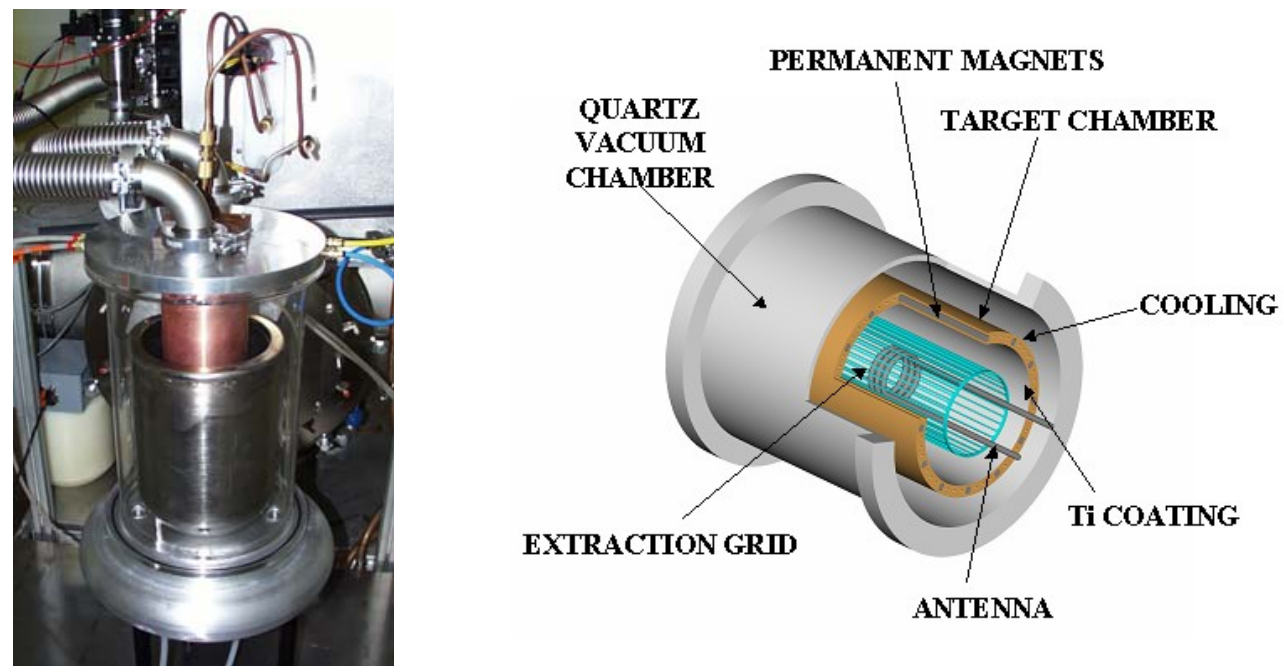

Figure 2: Perspective view of the compact neutron source (Right) and its testing setup (Left).

The ion beam impinges on a titanium coated copper or aluminum target where either the 2.45 $\mathrm{MeV}$ D-D or $14 \mathrm{MeV}$ D-T neutrons are generated. The target consists of an inner diameter titanium layer on the order of 1 micron thick, a layer of copper to conduct the heat away from the target, a cooling channel for water and a coil (or permanent magnet set), and an outer layer of stainless steel. The permanent magnets or the coil is used to provide a small magnetic field to keep electrons from being accelerated towards the plasma electrode after the deuterons impact the target surface. The deuteron beam is used both to load the target and to drive the D-D reaction. The target is loaded continuously with the beam, and therefore has a long lifetime. The target lifetime is limited by the sputtering of the titanium layer. This layer thickness can be increased for longer life, but at the expense of increased resistance to heat removal. It is 
necessary to keep the target temperature between 100 and $200{ }^{\circ} \mathrm{C}$ so that the deuterium remains at the surface and does not migrate below the target surface. The target is designed to withstand heat loading of at least $200 \mathrm{~W} / \mathrm{cm}^{2}$. This is only about one third of the heat flux that can be removed by water cooling without exceeding a target temperature of $150^{\circ} \mathrm{C}$. The outer diameter of the source is approximately $26 \mathrm{~cm}$. The source length is to be determined so that the target heat flux will not exceed $200 \mathrm{~W} / \mathrm{cm}^{2}$. For comparison, the $2 \times 10^{13}$ neutrons per second D-T neutron source developed by the Sandia National Laboratory (SNL) [4] has a target that can handle up to $4 \mathrm{~kW} / \mathrm{cm}^{2}$.

The choice of the ion accelerating voltage is dictated by the energy dependent fusion cross section along with practical limitations. Figure 3 shows that the optimal acceleration energy for D-T fusion is nearly $150 \mathrm{keV}$. The neutron yield from a 1 Amper current of mixed D and T ions accelerated to $150 \mathrm{keV}$ is estimated to be $5.9 \times 10^{13} \mathrm{D}-\mathrm{T}$ neutrons per second. This neutron source intensity is sufficient for BNCT treatment of deep-seated brain tumors; the needed treatment time was found to be less that one hour [2]. The corresponding heat loading on the target is 150 $\mathrm{kW}$. At a heat loading of $200 \mathrm{~W} / \mathrm{cm}^{2}$ the minimum required target surface area is $750 \mathrm{~cm}^{2}$. A cylindrical target that is $16 \mathrm{~cm}$ in diameter provides $\sim 50 \mathrm{~cm}^{2}$ surface area per $\mathrm{cm}$ length. Hence the required target length is only $15 \mathrm{~cm}$.

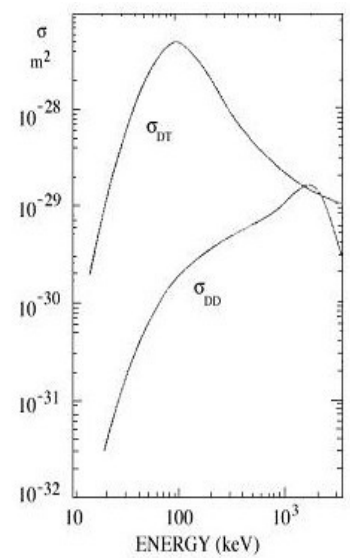

Figure 3: Energy dependent cross section for D-T and D-D fusion

For generating D-D neutrons it is energetically desirable to maximize the D beam energy up to the peak of the D-D cross section (See Figure 3). Unfortunately, it is difficult to work with voltages beyond $200 \mathrm{kV}$ because power supplies and high voltage components such as cables and connectors are not commercially available and fabricating high voltage components would significantly increase costs. With a $200 \mathrm{kV}$ extraction voltage and a $1 \mathrm{~A}$ beam current, this 
source produces an isotropic source of $2.45 \mathrm{MeV}$ neutrons at about $1.1 \times 10^{12}$ neutrons per second. Though the maximum extracted beam current from the deuterium source is approximately $27 \mathrm{~A}$, the size and cost of the power supply as well as the cost of electricity becomes excessive beyond $400 \mathrm{~kW}$, which corresponds to a $2 \mathrm{~A}$ current at $200 \mathrm{KeV}$. This will provide a neutron source intensity of $2.3 \times 10^{12} \mathrm{n} / \mathrm{s}$ and will require an effective target length of $40 \mathrm{~cm}$. This neutron source intensity is a factor of 30 or so short of the intensity needed for BNCT of deep brain tumors.

\section{METHODOLOGY}

The calculations were performed using the 3-D Monte Carlo N-Particle (MCNP) code version $4 \mathrm{C}$ [5]. It is a general-purpose, continuous-energy, generalized-geometry, and coupled neutron/photon/electron transport code created by Los Alamos National Laboratory and distributed by Oak Ridge National Laboratory. MCNP4C can simulate coupled neutrons/photons transport in complex 3-D geometries accurately accounting for space and energy dependence. Two different MCNP computational modes are used for the simulations: the eigenvalue (or KCODE) mode to calculate the multiplication factor of the SCM and a source mode to simulate the transport of neutrons and associated secondary photons from the compact neutron source to the brain and to calculate the dose to the tumor and to the normal tissue of the brain.

The human body is simulated using the MIRD-5 anthropomorphic model for MCNP, with a small cylinder $-1 \mathrm{~cm}$ in radius - along the axis of the brain divided in 26 different axial zones to evaluate the doses at different depths inside the brain. 3-D semitransparent view of the human head in the MIRD-5 model, with the small cylinder for the dose tallies is shown in Figure 4. The direction of the neutron beam is assumed inclined at 37.7 deg to the patient spine, when the patient rests on his/her back.

The dose to the tumor and to the healthy tissue is calculated using Equations (1) and (2) in which $D_{B}, D_{n}$ and $D_{\gamma}$ are, respectively, the contribution of ${ }^{10} B$, other neutron interactions and photons to the dose.

$$
\begin{aligned}
& \mathrm{D}_{\text {tot }}=\mathrm{D}_{\mathrm{B}} \mathrm{RBE}_{\mathrm{B}}+\mathrm{D}_{\mathrm{n}} \mathrm{RBE}_{\mathrm{n}}+\mathrm{D}_{\gamma} \mathrm{RBE} \gamma \\
& D_{B}=\int k_{B}(E) \phi_{n}(E) d E ; \quad D_{\gamma}=\int k_{\gamma}(E) E \phi_{\gamma}(E) d E ; \quad D_{n}=\int k_{n}(E) \phi_{n}(E) d E
\end{aligned}
$$




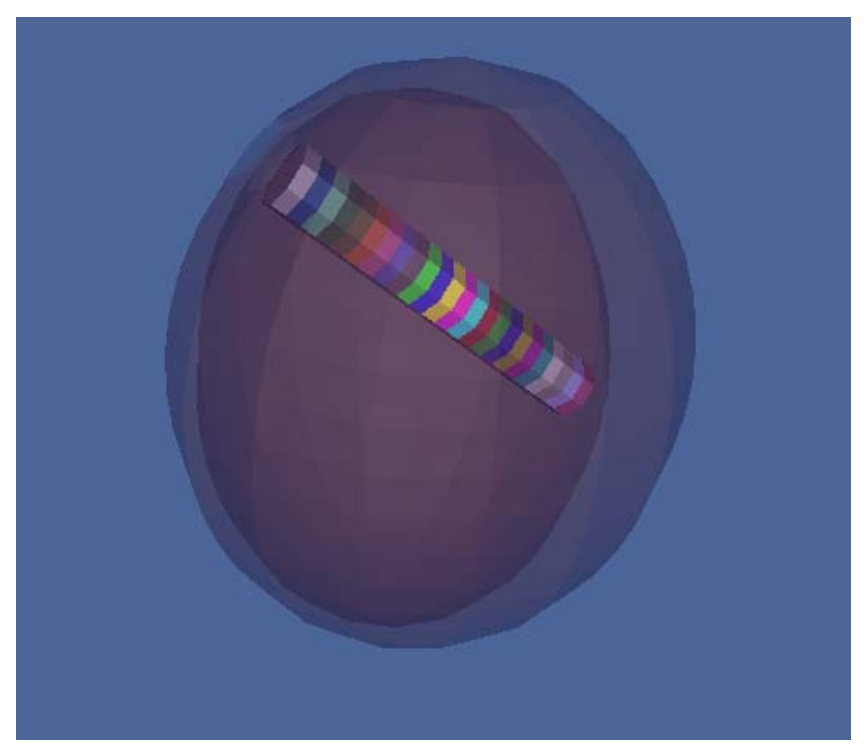

Figure 4: 3-D semitransparent view of the human head as modeled in MIRD-5: visible is the skull (external ellipsoid), the brain (the internal ellipsoid); the volumes over which dose tallies are calculated are the colored cylindrical volumes.

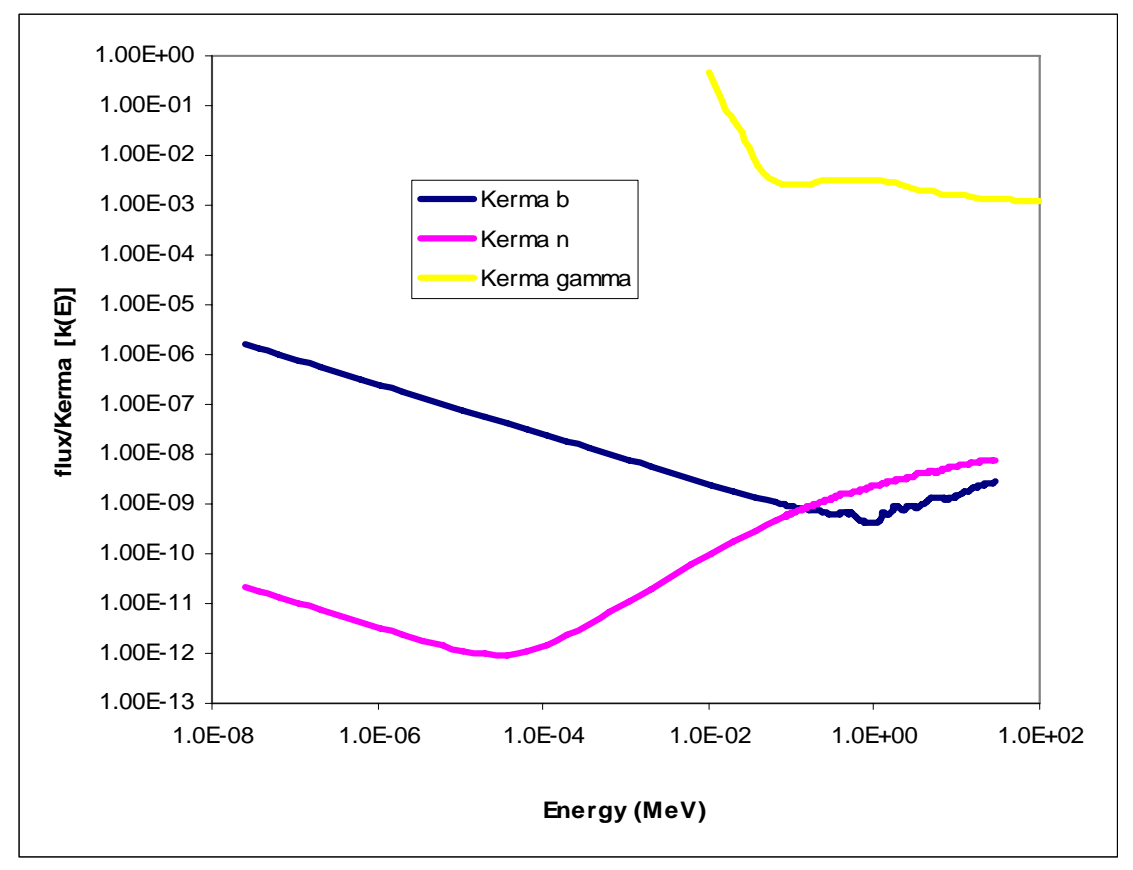

Figure 5: Fluence to kerma conversion factors as a function of energy.

$\mathrm{k}(\mathrm{E})$ is the fluence-to-dose conversion factors and $\mathrm{k}_{\gamma}$ is the photon mass attenuation coefficient.

$\phi_{x}(E)$ is the energy dependent fluence of either neutrons $(x=n)$ or photons $(x=\gamma)$. The boron, 
neutron and photon doses are weighted by their Relative Biological Effectiveness (RBE). The boron contribution to the dose is also weighted by the Compound Factor $\left(\mathrm{CF}_{\mathrm{B}}\right)$ that were factored in the respective RBE's. The $\mathrm{CF}_{\mathrm{B}}$ is different for healthy and tumor tissue.

Numerical values of all these terms are taken from references 1 and 2 . What limits the patient treatment time is the constraint of 12.5 Gy for the maximum dose to the healthy tissue ${ }^{1}$. This constraint also limits the maximum dose that can be delivered to the tumor. The tumor control dose, that is, the minimum dose required to kill the tumor is assumed to be $20 \mathrm{~Gy}$. The boron concentration assumed is 45 and $13 \mathrm{mg}{ }^{10} \mathrm{~B}$ per gram of, respectively, normal tissue and tumor ${ }^{1}$.

Three optimization studies were performed: optimization of the sub-critical neutron multiplier (SCM), optimization of the beam-shaping assembly (BSA) and optimization of the reflector. The SCM optimization objective is to maximize the current of neutrons that leak out from the SCM in the direction of the patient, without exceeding the maximum permissible $\mathrm{k}_{\mathrm{eff}}$. Minimizing the required uranium inventory is another objective. SCM design variables considered include the uranium enrichment level in the range not exceeding $20 \%{ }^{235} \mathrm{U}$ (for proliferation concerns), SCM shape and dimensions, fuel thickness and moderator thickness.

The objective of the BSA optimization is to maximize the tumor dose rate using the optimal SCM while maintaining a tumor-to-normal tissue dose ratio of at least 20 to 12.5 (corresponding to the tumor control dose and to the healthy tissue dose limit). The BSA design variables include its shape, dimensions and composition.

The reflector optimization is, in fact, an integral part of the SCM optimization and of the BSA optimization. The reflector design variables are composition and thickness.

The overall design objective is to get a treatment time that does not significantly increase beyond one hour when the effective multiplication factor of the SCM is $\mathrm{k}_{\mathrm{eff}}=0.98$.

\section{REFERENCE SYSTEM}

Figure 6 is an $\mathrm{x}-\mathrm{z}$ view of the BNCT facility found optimal last year ${ }^{2}$ as reported in last year's progress report.

\footnotetext{
${ }^{1}$ BNL clinical trial protocol sets the limit of 12.5 Gy for healthy tissue and no limit for the skin. Reddening has been observed at $8 \mathrm{~Gy}$ (limit not applied in this study).
} 


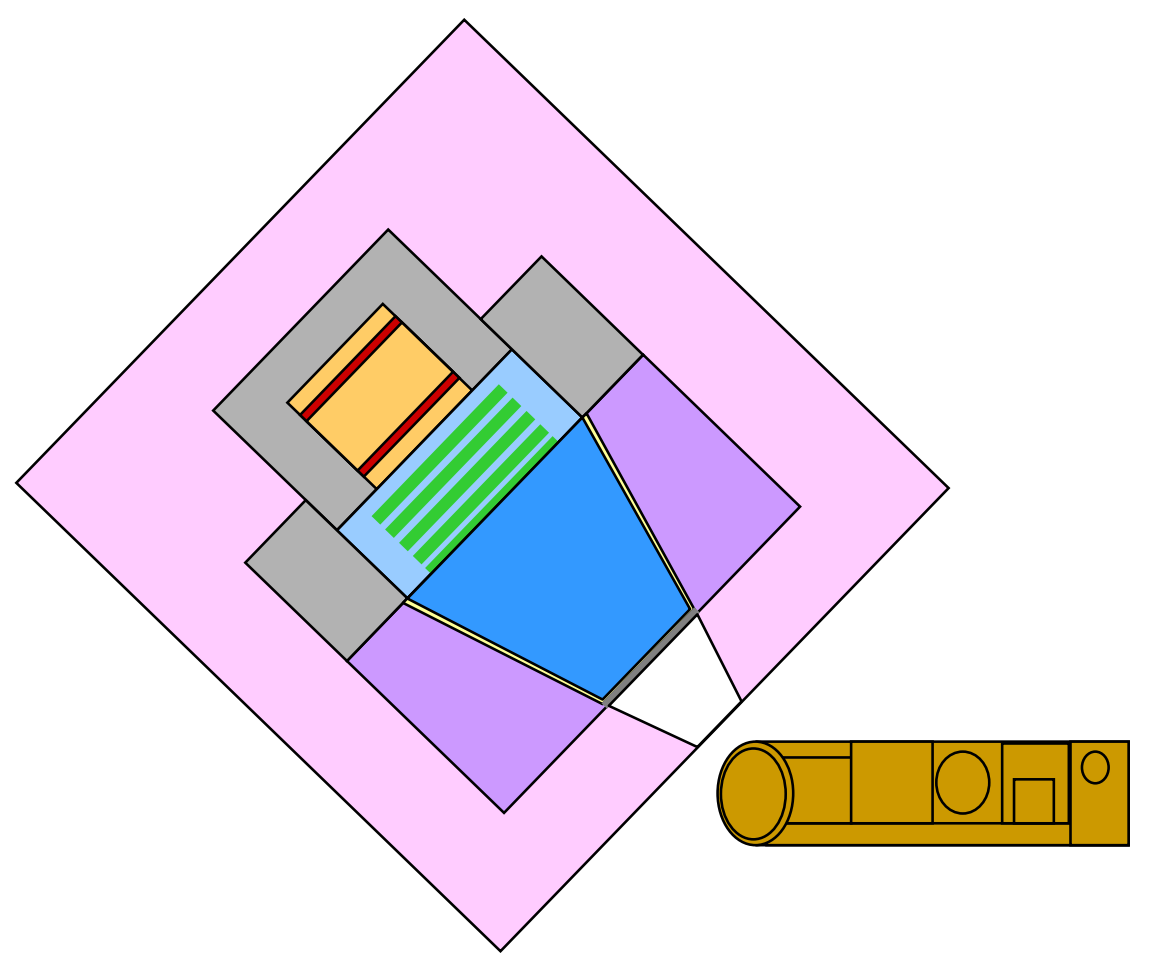

CNS

Cylindrical neutron source for MCNP

SCM water

Uranium plates

Variable BSA

${ }^{6} \mathrm{Li}$ filter

$\mathrm{Al}_{2} \mathrm{O}_{3}$ reflector

Lead reflector

Polyethylene delimiter

Beam port

Human phantom

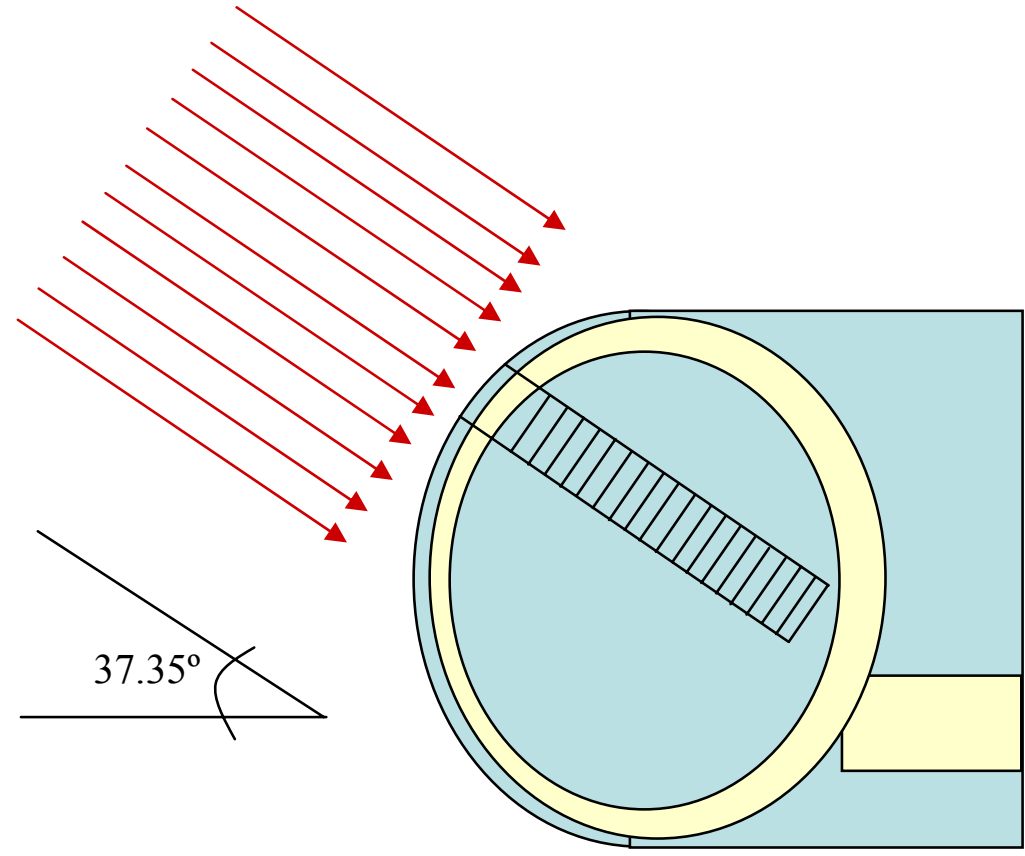

Figure 6: $\mathrm{x}-\mathrm{z}$ view of the reference BNCT facility arrived at in 2005 (top) and the neutron beam incident on MIRD-5 phantom head 
In this system the CNS is positioned vertically, the SCM is made of five $1 \mathrm{~cm}$ thick metallic-uranium disks spaced $2.5 \mathrm{~cm}$ apart. Table I gives the dimensions of the reference system, Table II gives the densities of the reference system. The uranium enrichment is $9 \%$ for the first three plates (i.e. towards the CNS) and 12\% for the last two (i.e. towards the BSA). The conical BSA is made of ${ }^{7} \mathrm{LiF}$ in the $30 \mathrm{~cm}$ facing the $\mathrm{SCM}$ and Fluental ${ }^{2}$ in the last $20 \mathrm{~cm}$ facing the brain. The dimensions of the BSA are shown in Table I: the truncated cone has the larger base towards the BSA and the smaller base towards the collimator and the brain. The angle of the cone is 14.9 degrees. The cladding of the uranium plates is neglected. The cost of the uranium for the SCM in this configuration is about $\$ 215,000$, assuming a unit cost of $29.2 \$ / 1 b$ of $U_{3} \mathrm{O}_{8}$ and $110 \$ /$ SWU.

Table I: Dimensions of the reference system

\begin{tabular}{|c|c|}
\hline \multicolumn{2}{|l|}{ CNS } \\
\hline $8.0965 \mathrm{~cm}$ & Cu cylinder (IR) \\
\hline $8.414 \mathrm{~cm}$ & Cu cylinder (OR), cooling channel (IR) \\
\hline $9.049 \mathrm{~cm}$ & Cooling channels (OR), outer skin (IR) (water) \\
\hline $9.2075 \mathrm{~cm}$ & Outer skin (OR) \\
\hline $12.495 \mathrm{~cm}$ & Quartz vacuum chamber (IR) \\
\hline $13.005 \mathrm{~cm}$ & Quartz vacuum chamber (OR) \\
\hline $20.955 \mathrm{~cm}$ & Length of the CNS \\
\hline $10 \mathrm{~cm}$ & Thickness of the $\mathrm{Pb}$ reflector \\
\hline \multicolumn{2}{|l|}{ SCM } \\
\hline $15 \mathrm{~cm}$ & U plates radius \\
\hline $20 \mathrm{~cm}$ & Water reflector radius \\
\hline $35 \mathrm{~cm}$ & Radial $\mathrm{Pb}$ reflector radius \\
\hline \multicolumn{2}{|l|}{ BSA } \\
\hline $30 \mathrm{~cm}$ & Thickness of the ${ }^{7}$ LiF part \\
\hline $20 \mathrm{~cm}$ & Thickness of the fluental part \\
\hline $35 \mathrm{~cm}$ & Outer radius of the alumina reflector \\
\hline $1 \mathrm{~mm}$ & ${ }^{6}$ LiF filter thickness \\
\hline $1 \mathrm{~mm}$ & Lead filter thickness \\
\hline $6 \mathrm{~cm}$ & Radius of the collimator \\
\hline \multicolumn{2}{|c|}{ Outer lithiated polyethylene shield } \\
\hline \begin{tabular}{r|l|}
15 & $\mathrm{~cm}$ \\
\end{tabular} & Thickness of the front polyethylene shield \\
\hline $3 \mathrm{~cm}$ & Thickness of the frontal lead shield \\
\hline $20 \mathrm{~cm}$ & Thickness of the back polyethylene shield \\
\hline $55 \mathrm{~cm}$ & Polyethylene shield radius \\
\hline
\end{tabular}

\footnotetext{
${ }^{2}$ Fluental: $\mathrm{Al} / \mathrm{AlF}_{3}(40 \% / 60 \%)$
} 
Table II: Material densities of the reference system

\begin{tabular}{|c|c|}
\hline & Densities \\
\hline U metal & $19.05 \mathrm{~g} / \mathrm{cc}$ \\
\hline Water & $1 \mathrm{~g} / \mathrm{cc}$ \\
\hline Lead & $11.34 \mathrm{~g} / \mathrm{cc}$ \\
\hline Alumina & $3.69 \mathrm{~g} / \mathrm{cc}$ \\
\hline${ }^{7}$ LiF & $2.635 \mathrm{~g} / \mathrm{cc}$ \\
\hline Fluental & $2.74 \mathrm{~g} / \mathrm{cc}$ \\
\hline Lithiated polyethylene $^{3}$ & $0.9843 \mathrm{~g} / \mathrm{cc}$ \\
\hline${ }^{6}$ LiF & $2.6 \mathrm{~g} / \mathrm{cc}$ \\
\hline
\end{tabular}

Figure 7 shows the total dose to the healthy tissue and to the tumor as a function of the cell number that denotes the depth into the brain. The maximum tumor dose of $47 \mathrm{~Gy}$ is reached at about $2.5 \mathrm{~cm}$ depth with an irradiation time of about 25.6 hours. The lethality range, that is, the range over which the dose exceeds the tumor control dose of $20 \mathrm{~Gy}$, is from $0 \mathrm{~cm}$ to about $7 \mathrm{~cm}$. Figure 8 shows the ratio between the dose to the tumor to the dose to the healthy tissue, also denoted as $\mathrm{H} / \mathrm{L}$; it peaks at around $3 \mathrm{~cm}$ depth at about 4.

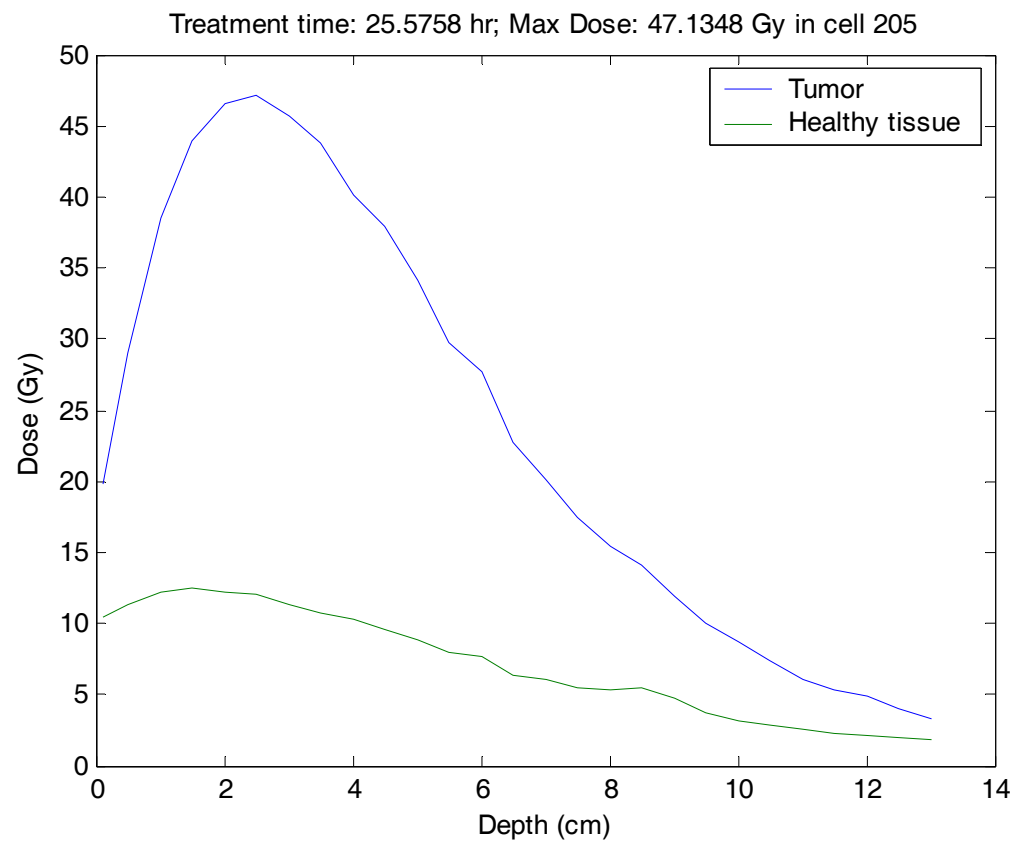

Figure 7: Total dose to the healthy tissue and to the tumor as a function of depth into the brain. Goff's final setup

${ }^{3}$ Lithiated polyethylene atomic fraction: ${ }^{1} \mathrm{H} 0.5262 ; \mathrm{C} 0.2969 ;{ }^{16} \mathrm{O} 0.1007 ;{ }^{6} \mathrm{Li} 0.0724 ;{ }^{7} \mathrm{Li} 0.0038$. 
The above design is not satisfactory because the required treatment time is significantly longer than the one hour design objective even with a SCM that is unrealistically close to criticality $-\mathrm{k}_{\text {eff }}=0.99193 \pm 0.0006$.

In the following we describe a new thrust for optimizing the BNCT system with the goal of establishing whether or not it is possible to use the $10^{12} \mathrm{n} / \mathrm{s}$ D-D compact neutron source interfaced with a small $\mathrm{k}_{\text {eff }}=0.98 \mathrm{SCM}$ to achieve an intense enough neutron beam for effectively treating brain tumors in approximately 1 hour. The best design we came up with needs a 3.4 hours irradiation time when $\mathrm{k}_{\mathrm{eff}}$ is 0.98 . This is an 18 -folds improvement relative to the previous optimal design. Although the neutron multiplication is short of the initial design goal by a factor of 3.4, we cannot preclude this CNS/SCM combination from being feasible. For example, it may turn out that the CNS could be designed to deliver nearly $5 \times 10^{12} \mathrm{n} / \mathrm{s}$ implying a treatment time of less than one hour.

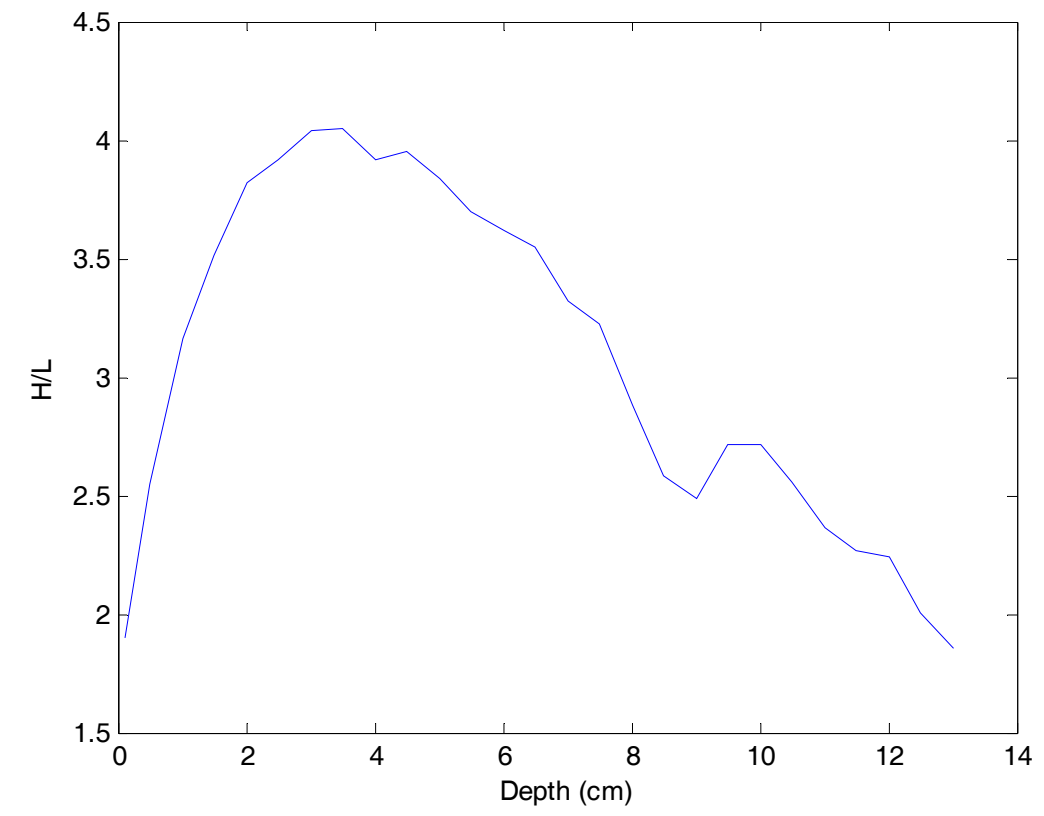

Figure 8: H/L (Tumor-to-healthy tissue dose ratio) in Goff's final setup 


\section{SCM OPTIMIZATION STUDIES}

\subsection{Cylindrical Sub-Critical Neutron Multiplier (SCM) optimization}

In this and the following sections the $\mathrm{k}_{\text {eff }}$ will be kept at 0.992 to compare properly with the design in Goff's study. Later we will switch back to $\mathrm{k}_{\mathrm{eff}}=0.98$, which is a more realistic value. The number of neutrons multiplied by the SCM (M) in first approximation depends only on $\mathrm{k}_{\text {eff: }}$ :

$$
\mathrm{M}=1+\mathrm{k}_{\mathrm{eff}}+\mathrm{k}_{\mathrm{eff}}{ }^{2}+\mathrm{k}_{\mathrm{eff}}{ }^{3}+\ldots=1 /\left(1-\mathrm{k}_{\mathrm{eff}}\right) \text {. }
$$

Additionally, by definition, $\mathrm{k}_{\mathrm{eff}}=\mathrm{k}_{\infty} * \mathrm{P}_{\mathrm{NL}}$, where $\mathrm{P}_{\mathrm{NL}}$ is the non-leakage-probability. To maximize the effectiveness of the SCM, we want the highest leakage probability, $\mathrm{P}_{\mathrm{L}}$, in the direction of the patient. To accomplish this we first have to find the highest possible $\mathrm{k}_{\infty}$ with the constraint of $\mathrm{k}_{\mathrm{eff}}=0.992$ and enrichment less or equal to $20 \%$ for proliferation concerns.

The optimal SCM configuration was found with the help of SWAN [3] and finalized by MCNP and TRITON/NEWT [6,7]. A $0.05 \mathrm{~cm}$ thick aluminum clad, not accounted for in the

previous analysis, was included on both sides of the uranium slabs. The design variables were the uranium thickness and the water gap thickness between the fuel plates. All the regions surrounding the SCM had the dimensions and composition as of the reference design shown in Figure 6. With the exception of the SCM, all the dimensions of this new system are specified in Table I and the densities in Table II.

The optimization process was performed in the following way: we started from a first guess on the geometry obtained by SWAN - fuel thickness of $0.06 \mathrm{~cm}$ and water thickness of $0.720 \mathrm{~cm}$. We then used MCNP to simulate a representative unit cell with reflective boundary conditions to confirm/ refine the SWAN first guess. Initially we kept the uranium thickness constant and found the water spacing that gives the highest possible $\mathrm{k}_{\text {inf }}$-slightly higher at $0.84 \mathrm{~cm}$ (see Figure 9 for the water thickness parametric study); then we kept the ratio between the water and fuel volumes at the optimal value and scaled the uranium thickness correspondingly. 


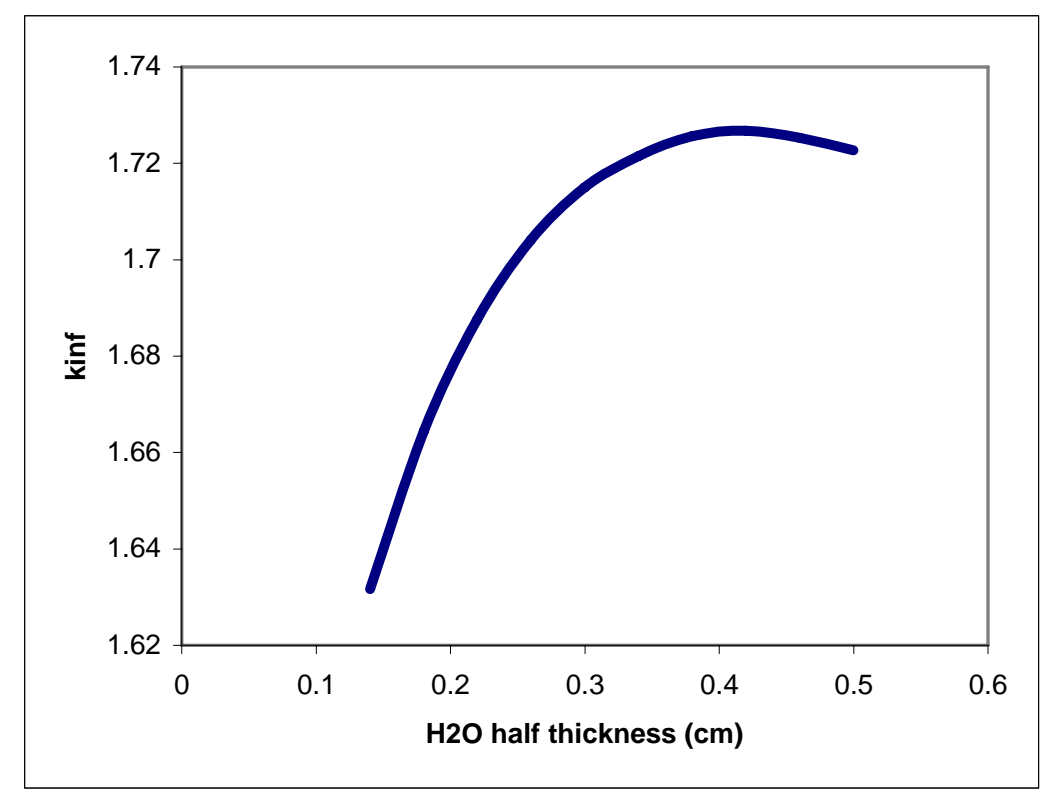

Figure 9: $\mathrm{k}_{\text {inf }}$ as a function of the $\mathrm{H}_{2} \mathrm{O}$ thickness for a system of $\mathrm{U}_{20 \%}$ metallic $0.06 \mathrm{~cm}$ thick and $0.05 \mathrm{~cm} \mathrm{Al}$ clad on both sides

The resulting highest $\mathrm{k}_{\infty}$ system was found made of $0.06 \mathrm{~cm}$ thick metallic $20 \%$ enriched $\mathrm{U}$ slab enclosed in aluminum cladding of $0.05 \mathrm{~cm}$ on both sides and with $0.84 \mathrm{~cm} \mathrm{H}_{2} \mathrm{O}$ between the fuel slabs (see Figure 10). The volume that in the previous study contained five one-centimeter-thick fuel slabs of lower enrichment uranium could exactly fit 17 optimal-geometry slabs.

The optimal $\mathrm{k}_{\infty}$ is 1.7267 versus 1.3612 in the previous reference model (Figure 5). Table III compares the leakage probability in the different directions: one facing the CNS, one facing the BSA and the side surface. The $\mathrm{k}_{\text {inf }}$ calculated by studying a representative unit cell, as we did here, does not account for the spectrum alteration induced by the insertion of the SCM in the real system. This effect is particulrly pronounced in this study, because of the small dimentions of the SCM and its high surface to volume ratio. Therefore, theoretically the $\mathrm{k}_{\text {inf }}$ optimization study should have been re-done each time a change was made to any part of the system such as reflectors, geometry, composition of the BSA etc. For practrical purposes we kept the optimal unit cell geometry throughout the study. 


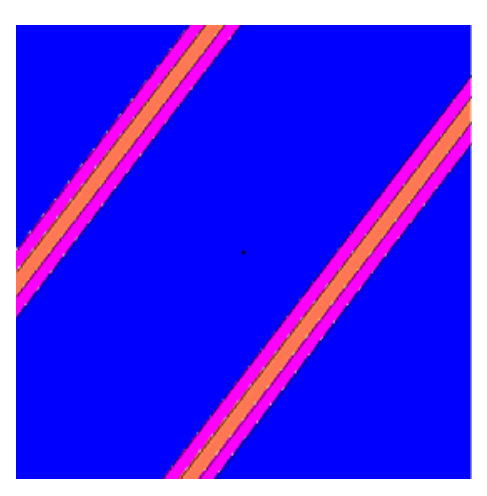

Figure 10: Details of the optimal $\mathrm{U}_{20 \%}+\mathrm{H}_{2} \mathrm{O}$ slab configuration inside the SCM. The uranium slabs (orange) are $0.06 \mathrm{~cm}$ thick; the aluminum cladding (pink) is $0.05 \mathrm{~cm}$ and the water (blue) is $0.84 \mathrm{~cm}$ thick. .

Table III: Net PL (leakage probabilities) for the reference Goff model and for the thin slab model from the three surfaces: CNS, BSA and the side surface. The PL is higher in the side direction.

\begin{tabular}{|c|c|c|}
\hline Surface & Reference SCM & Optimal SCM \\
\hline BSA & 0.1257 & 0.1478 \\
\hline Radial & 0.1285 & 0.1850 \\
\hline CNS & 0.1317 & 0.1679 \\
\hline Total & 0.3860 & 0.5007 \\
\hline
\end{tabular}

This implies that in the new SCM design the number of neutrons that will leak out per fission neutron is $17 \%$ higher than in the previous reference design. This makes the new SCM a more effective source of neutrons for our application.

Additionally the new SCM design needs substantially lower amount of uranium; the cost of the required amount of uranium reduces from $\$ 215,000$ of the reference design to $\$ 91,000$, despite of the higher cost for the higher enrichment - $20 \%$ versus $9 \%$ for $60 \%$ and $12 \%$ for $40 \%$ of the uranium.

Using the newly optimized SCM the maximum dose of about 43 Gy is achieved at about 3 $\mathrm{cm}$ depth as shown in Figure 11. The required irradiation time is 18.6 hours; this is the time it takes for the maximum healthy tissue dose, also shown in Figure 10, to reach the 12.5 Gy constraint. The lethality range is from 0 to $6.5 \mathrm{~cm}$. Figure 12 gives the tumor $(\mathrm{H})-$ to - healthy tissue (L) dose ratio as a function of depth in the brain. This is an improvement to the reference 24 hours treatment time. 
Table III also indicates that the largest leakage probability is in the side direction, which is undesirable: to improve the SCM performance we should maximize the net leakage in the BSA direction.

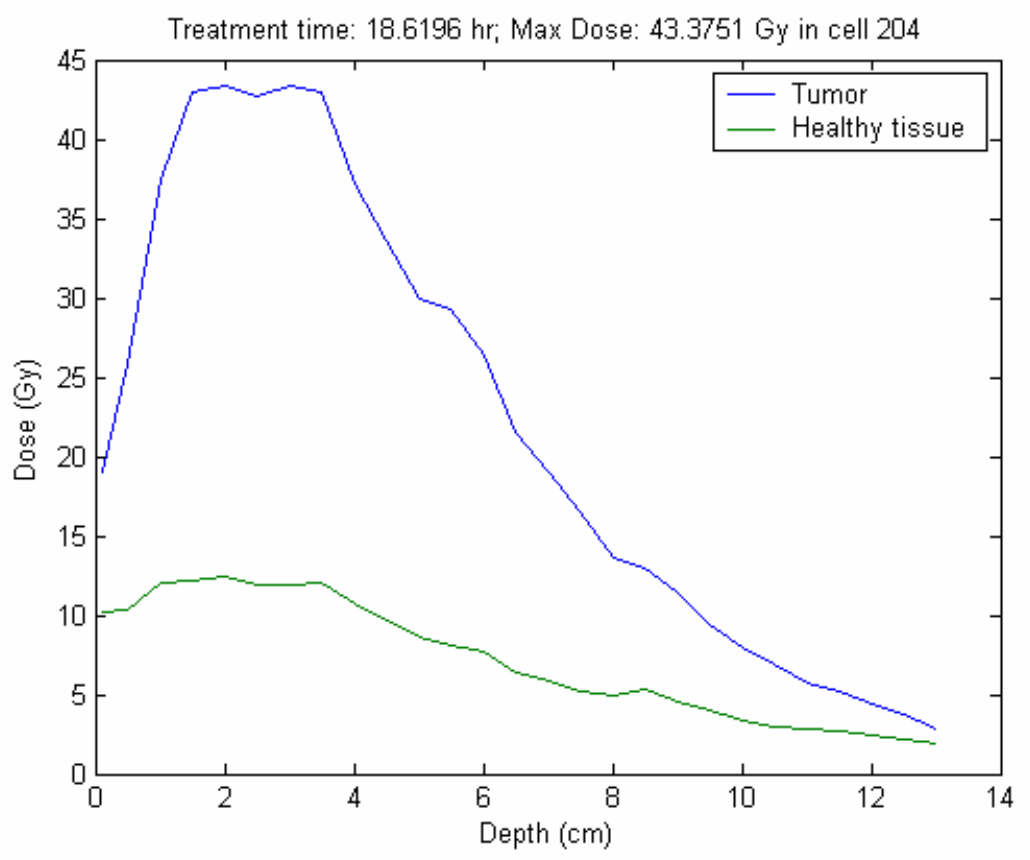

Figure 11: Total dose to the healthy tissue and to the tumor as a function of depth 


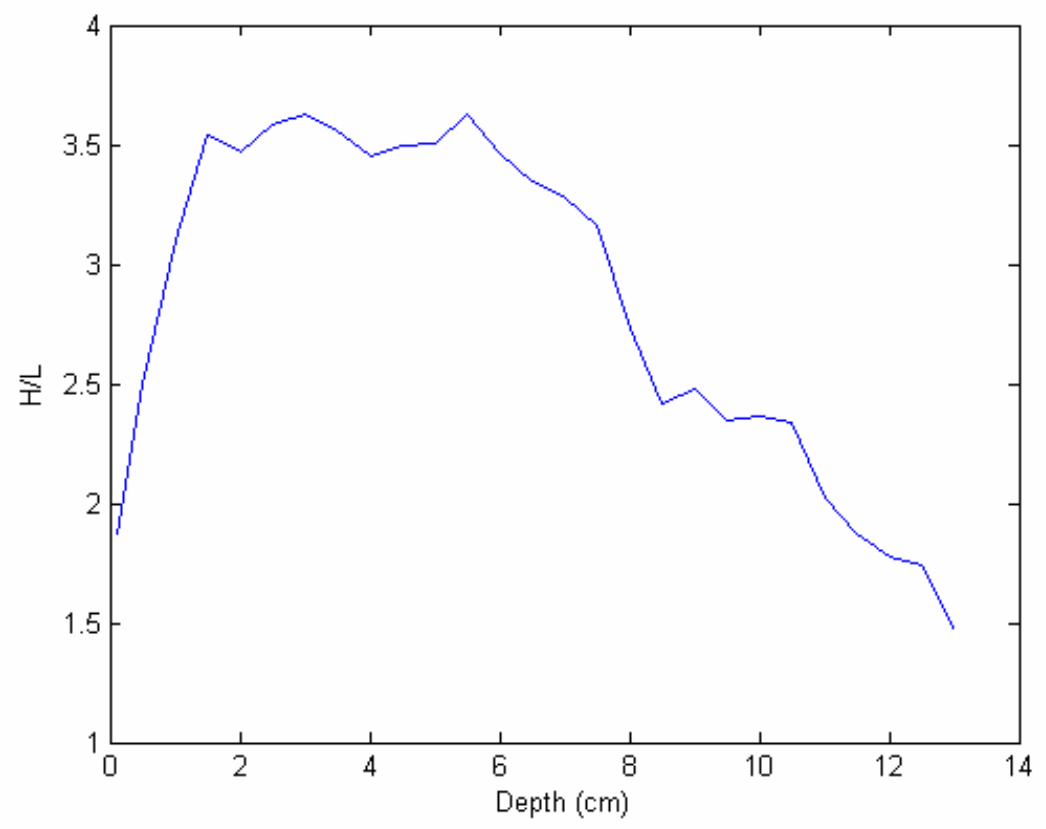

Figure 12: Tumor-to-healthy tissue dose ratio (H/L)

\subsection{Improvement of the SCM reflector}

To minimize the radial leakage from the $\mathrm{SCM}$ we need a very good reflector on the side $\mathrm{BeO}$ is tries instead of the lead reflector of the reference design. The choice of $\mathrm{BeO}$ is based on a companion reflector study that is summarized in Appendix A.

Figure 13 shows the BNCT facility considered in which $\mathrm{BeO}$ substitutes $\mathrm{Pb}$ on all sides, including around the CNS. The thickness is kept the same as in the $\mathrm{Pb}$ case (see Table I for the dimensions of the $\mathrm{Pb}$ reflector). Around the SCM the outer radius is kept as in the reference design at $35 \mathrm{~cm}$, but the water reflector has been substituted with $\mathrm{Be}$, while the outer radius of the $U$ disks is adjusted to obtain the desired level of $k_{\text {eff. }}$ The improved reflection resulted in an increase in the multiplication factor that was compensated by reducing the size of the SCM. So as to maximize the axial leakage probability SCM core radius was increased from $15 \mathrm{~cm} \mathrm{~cm}$ to $19.9 \mathrm{~cm}$ while its thickness was reduced from $17 \mathrm{~cm}$ to $9 \mathrm{~cm}$. As a result the number of fuel plates was reduced from 17 to 9 while $\mathrm{k}_{\text {eff }}$ was maintained at its reference value of 0.992 . The reduced amount of uranium resulted in extra saving in the total uranium cost, which for this case is estimated at $\$ 86,000$. 
Figure 14 and Figure 15show the resulting dose distributions in the brain: the maximum dose is about $41.33 \mathrm{~Gy}$; it is achieved in about 9.5 hours of irradiation time at a depth of about $2 \mathrm{~cm}$ inside the brain.

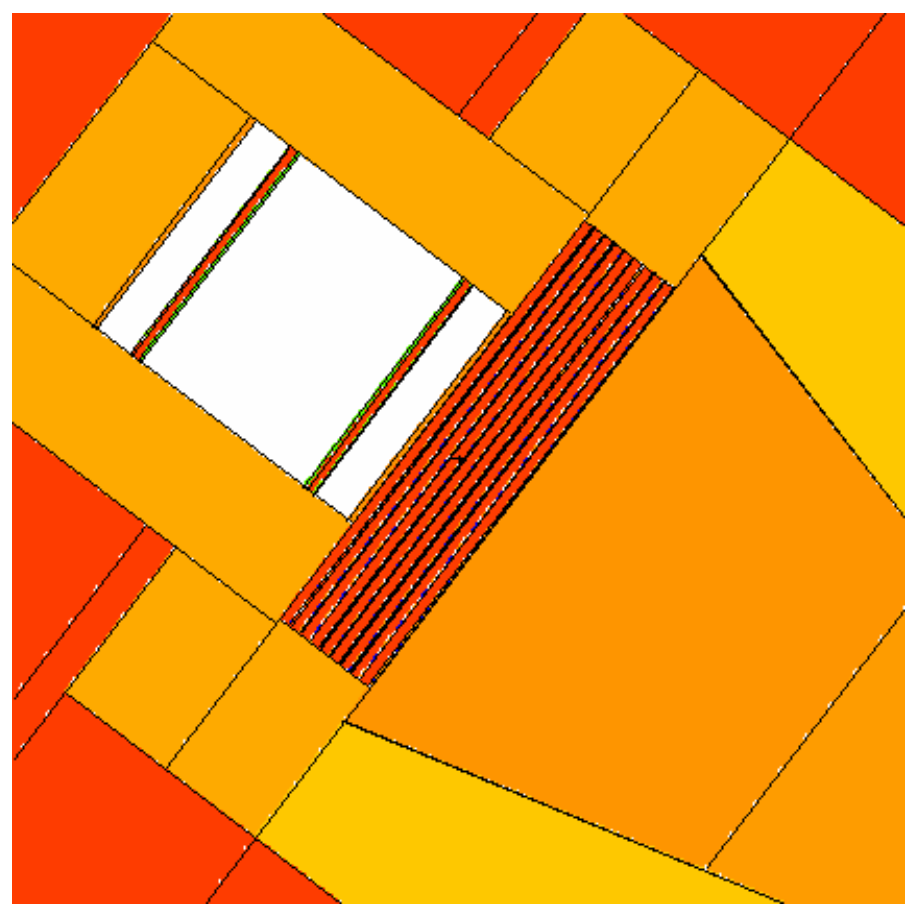

Figure 13: $\mathrm{x}-\mathrm{z}$ view of the system with the improved reflector: the color represent the density of the materials in the system, from the orange for the lighter to blue for the heavier like uranium.

The light orange around the $\mathrm{CNS}$ is $\mathrm{BeO}$, the orange around it is lithiated polyethylene, the uranium slabs are nine. The orange with a conical shape on the right is the BSA; the yellow around it is the alumina reflector. 


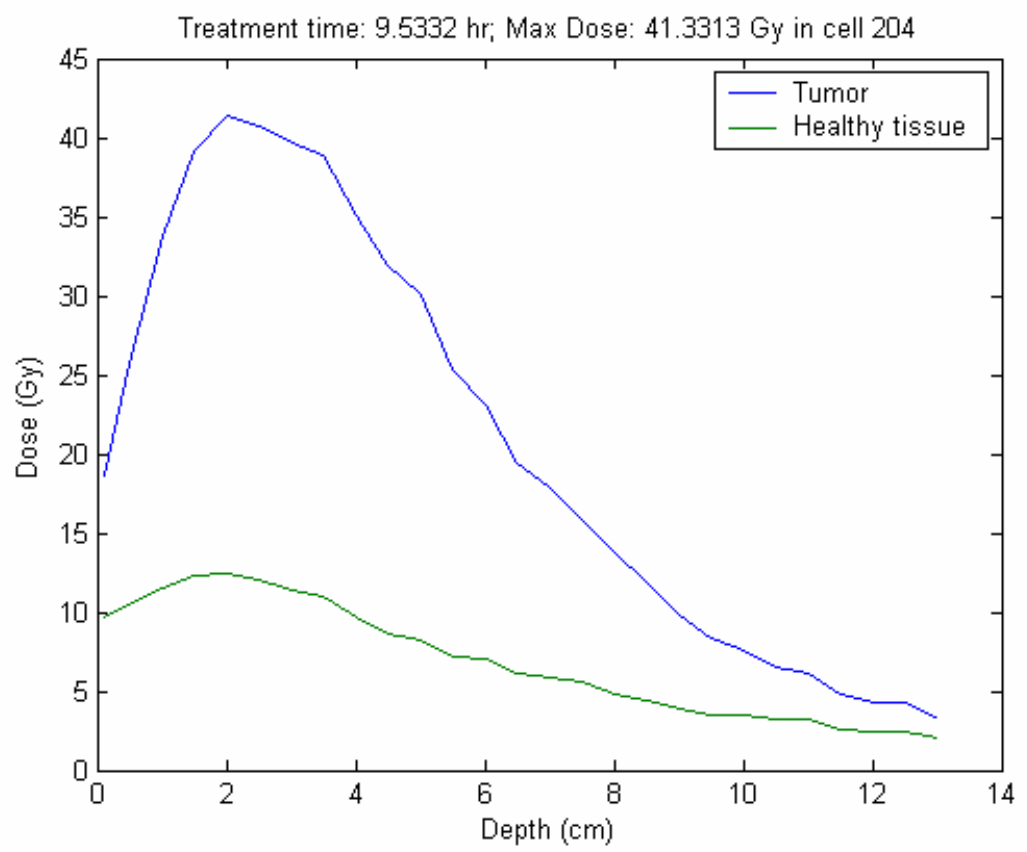

Figure 14: Total dose to the healthy tissue and to the tumor as a function of depth

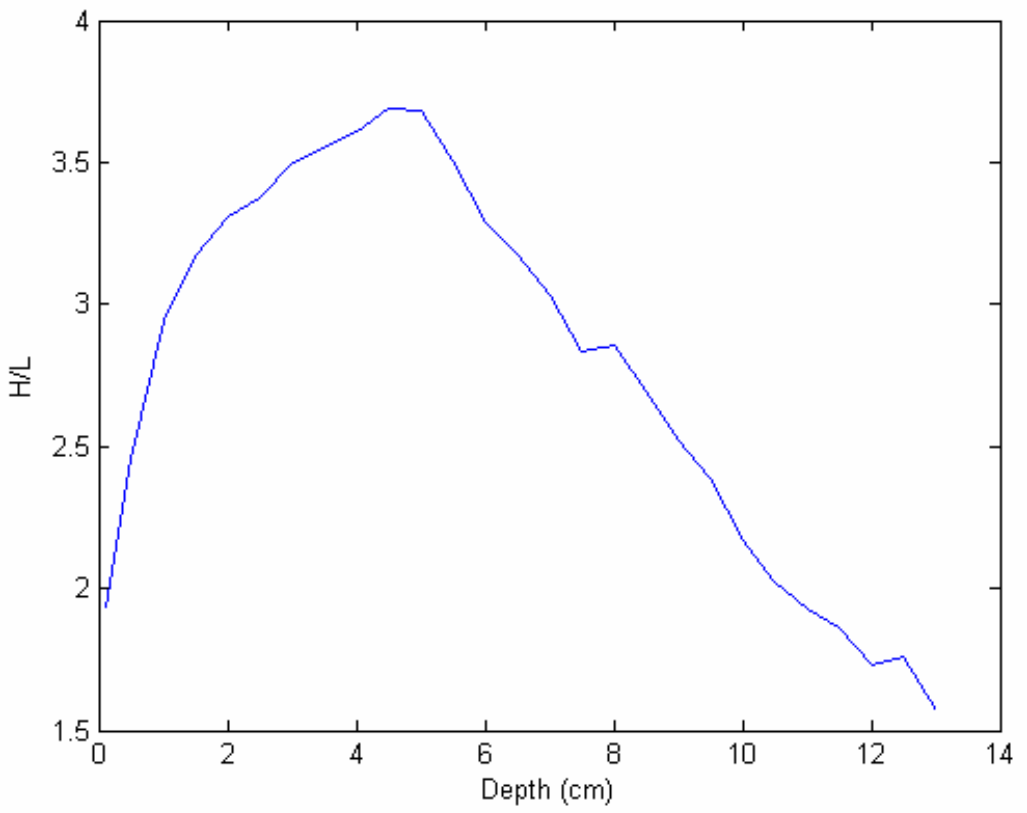

Figure 15: Tumor-to-healthy tissue dose ratio $(\mathrm{H} / \mathrm{L})$ 


\subsection{Increasing the source effectiveness}

Despite of the very effective $\mathrm{BeO}$ reflector around both the CNS and the SCM, the fraction of neutrons generated by the CNS that reach the SCM in the previously considered configuration is only about $40 \%$. In order to improve the "source utilization factor" or source effectiveness, defined as the fraction of neutrons emitted by the CNS that reach the sub-critical multiplier, the SCM geometry was modified so as to increase the solid angle by which the SCM "views" the CNS.

Figure 16 shows the re-designed SCM; it has a "cup" shape that surrounds the CNS on three sides. The CNS, orientated axially, is surrounded by 2 cylinders of aluminum-clad $\mathrm{U}_{20 \%}$ fuel having the same water-to-fuel volume ratio as of the original optimal SCM described in Section 5.1. The number of vertical plates is reduced from 9 to 6 and their radius is adjusted to $17.45 \mathrm{~cm}$ to achieve a $\mathrm{k}_{\text {eff }}$ of 0.992 . This also resulted in further reduction of the uranium inventory bringing down the estimated uranium cost to about $\$ 74,500$.

The dose results for this design are shown in Figure 17, where the maximum dose of about $46.38 \mathrm{~Gy}$ is delivered at a depth of $2-4 \mathrm{~cm}$ with an irradiation time of 6.8 hours. The lethality range, or the region where the dose to the tumor is higher than $20 \mathrm{~Gy}$ is between 0 and $7 \mathrm{~cm}$. The $\mathrm{H} / \mathrm{L}$ ratio is shown in Figure 18; its peak of about 3.7 is at about $3.5 \mathrm{~cm}$ depth. 


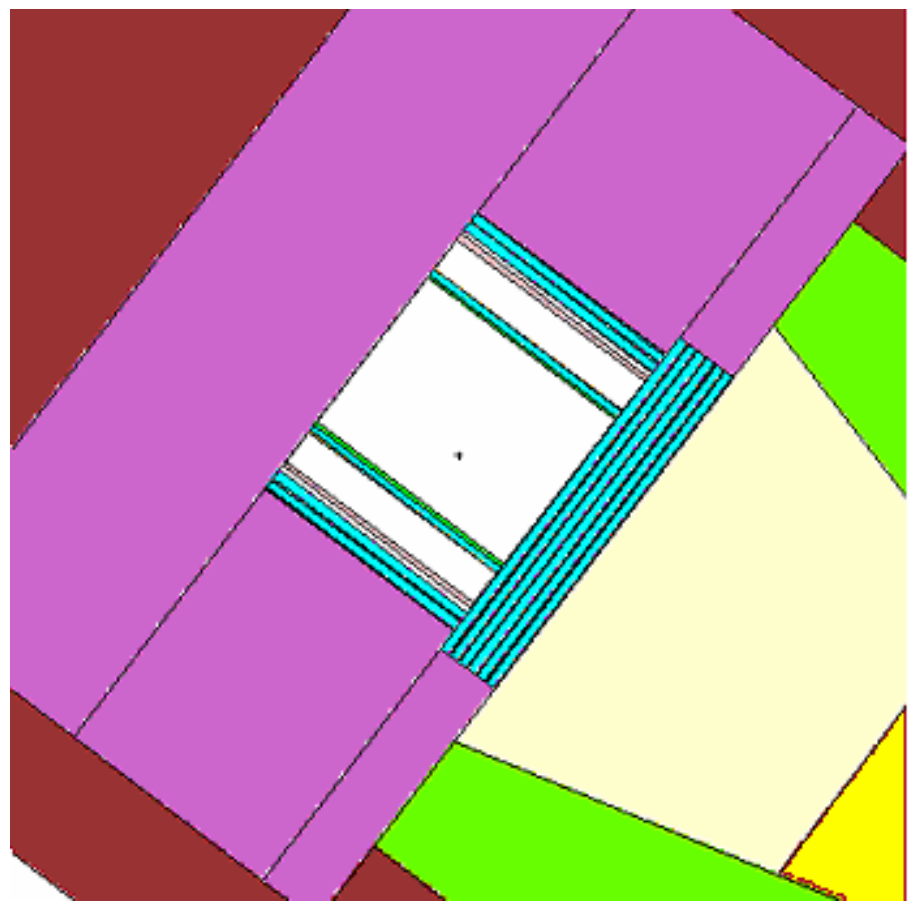

Figure 16: $\mathrm{x}-\mathrm{z}$ view of the system with improved source utilization. The source is encased in the $\mathrm{SCM}$, using 2 co-axial cylinders along the source, and 6 vertical plates. The violet is the $\mathrm{BeO}$ reflector surrounding both the CNS and the SCM.

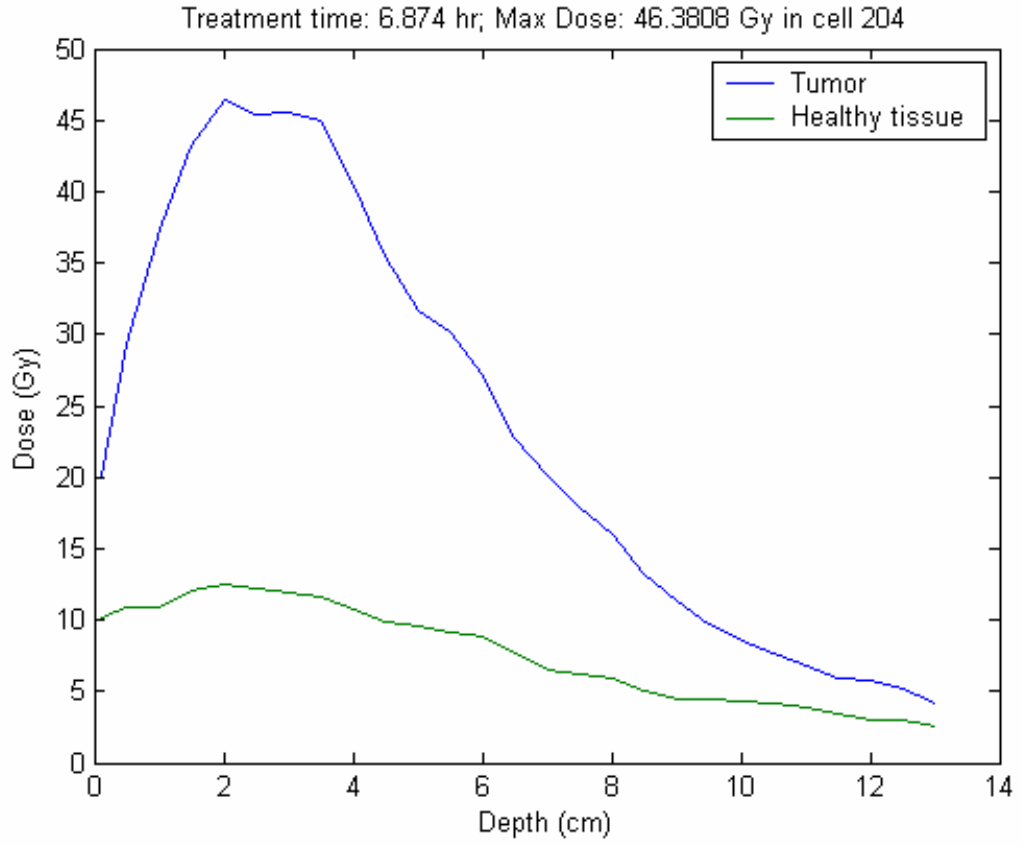

Figure 17: Total dose to the healthy tissue and to the tumor as a function of depth 


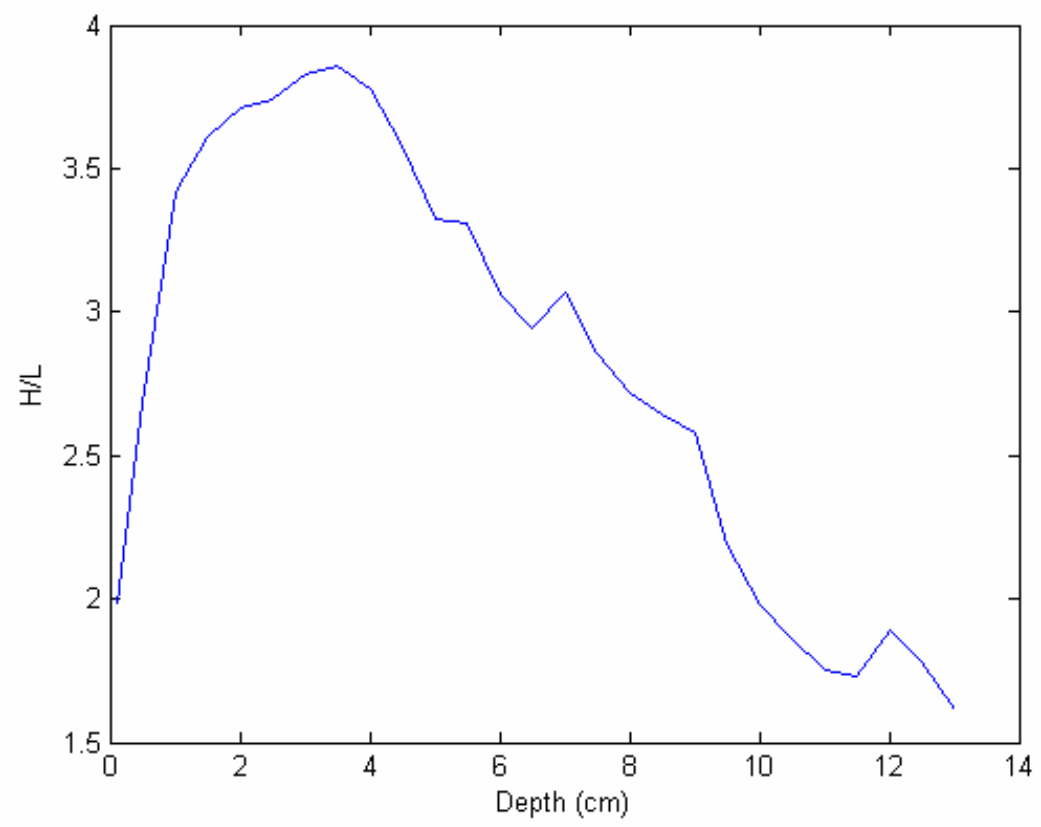

Figure 18: Tumor-to-healthy tissue dose ratio $(\mathrm{H} / \mathrm{L})$

\subsection{Further reflector thickening}

The thickness of the $\mathrm{BeO}$ reflector considered so far, in the vicinity of $20 \mathrm{~cm}$, is not sufficient for approaching the performance of an infinite reflector. For the reflector to approach the performance of an infinite reflector, its thickness should be approximately twice the thermal neutrons diffusion length $(\mathrm{L})$, which for $\mathrm{BeO}$ is about $31 \mathrm{~cm}$. This is verified in Figure 19 that shows $\mathrm{k}_{\mathrm{eff}}$ dependence on the reflector thickness. In the following a $62 \mathrm{~cm}$ thick $\mathrm{BeO}$ reflector is used. The resulting BNCT facility is shown in Figure 20 where the $\mathrm{BeO}$ reflector is in pink. Additional modification is an insertion of an extra layer of lead surrounding the alumina reflector of the BSA, shown violet in the Figure. The extra layer of lead was added to fill the space and was chosen because of the good reflective properties towards fast neutrons. The total thickness of the alumina plus the lead equals the thickness of the $\mathrm{BeO}$ reflector. Additionally, the ${ }^{6} \mathrm{LiF}$ filter surrounding the BSA in the part close to the SCM was eliminated. The original function of this layer was to reduce the current of thermal neutrons that leak from the BSA into the alumina reflector, where they generate unwanted capture gamma rays. However, the proximity of ${ }^{6} \mathrm{Li}$ to the SCM significantly degrades the reflector performance thus penalizing $\mathrm{k}_{\mathrm{eff}}$. 


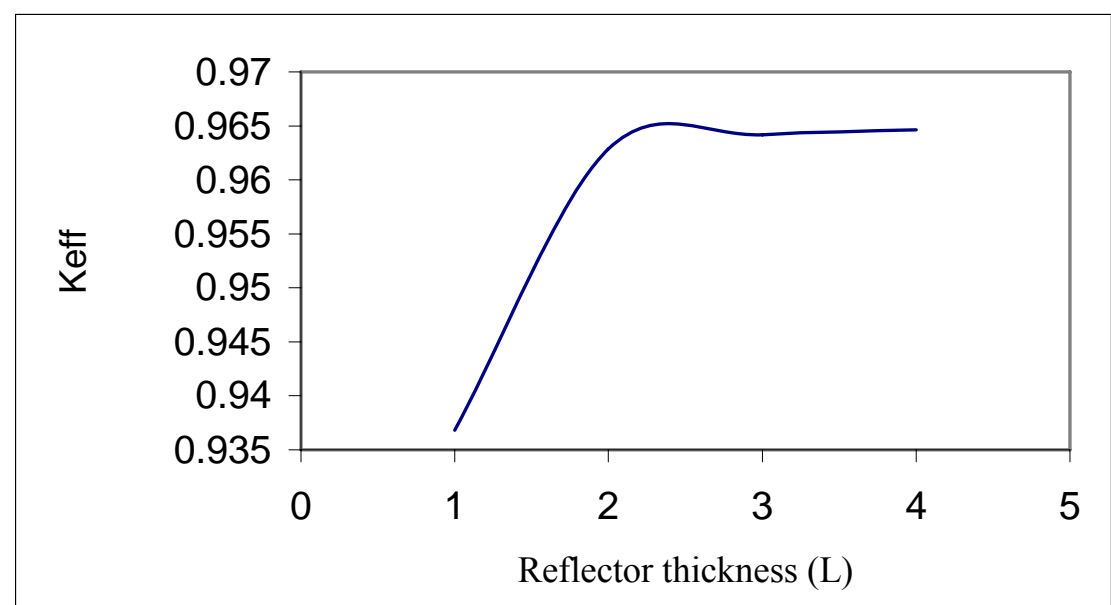

Figure 19: $\mathrm{k}_{\mathrm{eff}}$ as a function of Be reflector thickness in units of the thermal diffusion length (L)

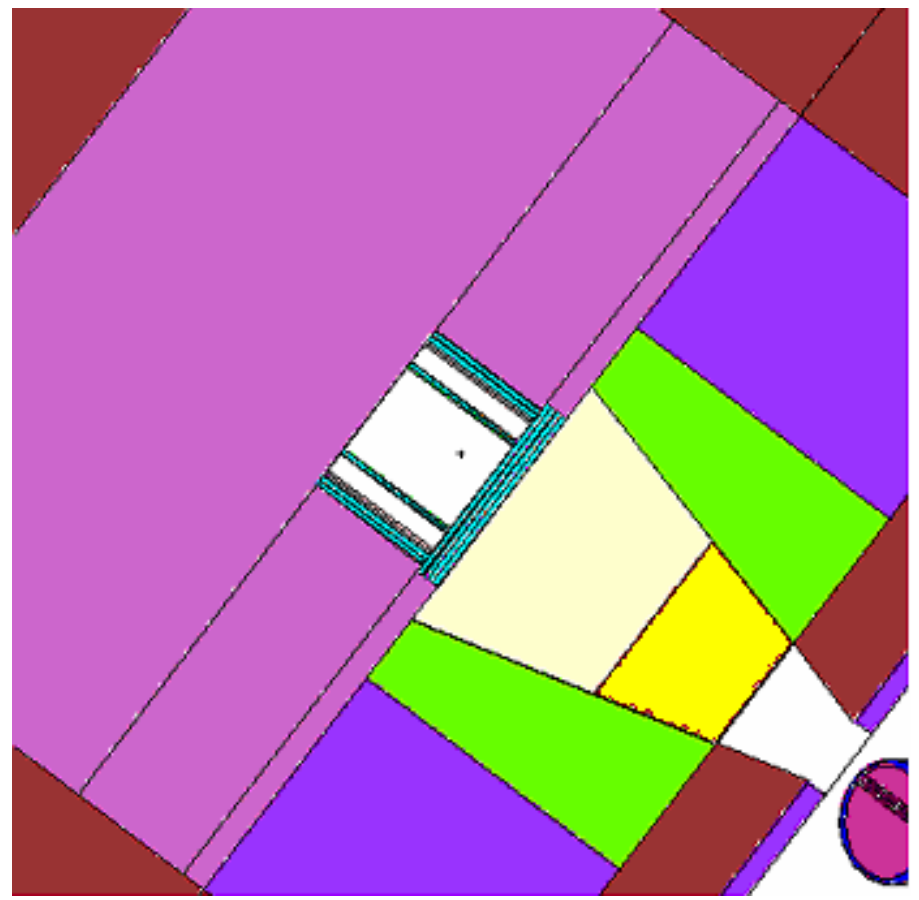

Figure 20: $\mathrm{x}-\mathrm{z}$ view of the system with improved reflector: the thickness of the $\mathrm{BeO}$ (in pink) is brought to $62 \mathrm{~cm}$, an extra layer of $\mathrm{Pb}$ (in violet) is added surrounding the alumina reflector of the BSA, the ${ }^{6} \mathrm{LiF}$ layer between the BSA and the alumina reflector is eliminated in the first part.

With the resulting substantial improvement in the reflector it became possible to further reduce the size of the SCM for the same $\mathrm{k}_{\mathrm{eff}}$. The horizontal cylinders surrounding the CNS 
axially are left unchanged but the number of vertical plates is reduced from 6 to 4 , and the radius is adjusted to $17.61 \mathrm{~cm}$ to match the $\mathrm{k}_{\mathrm{eff}}$ of 0.992 . The total $\mathrm{U}$ cost drops to $\$ 60,300$.

The dose results for this design are shown in Figure 21 and in Figure 22; the maximum dose of about $37 \mathrm{~Gy}$ is reached in 3.5 hours at about $2 \mathrm{~cm}$ depth while H/L peaks at $\sim 4 \mathrm{~cm}$ depth.

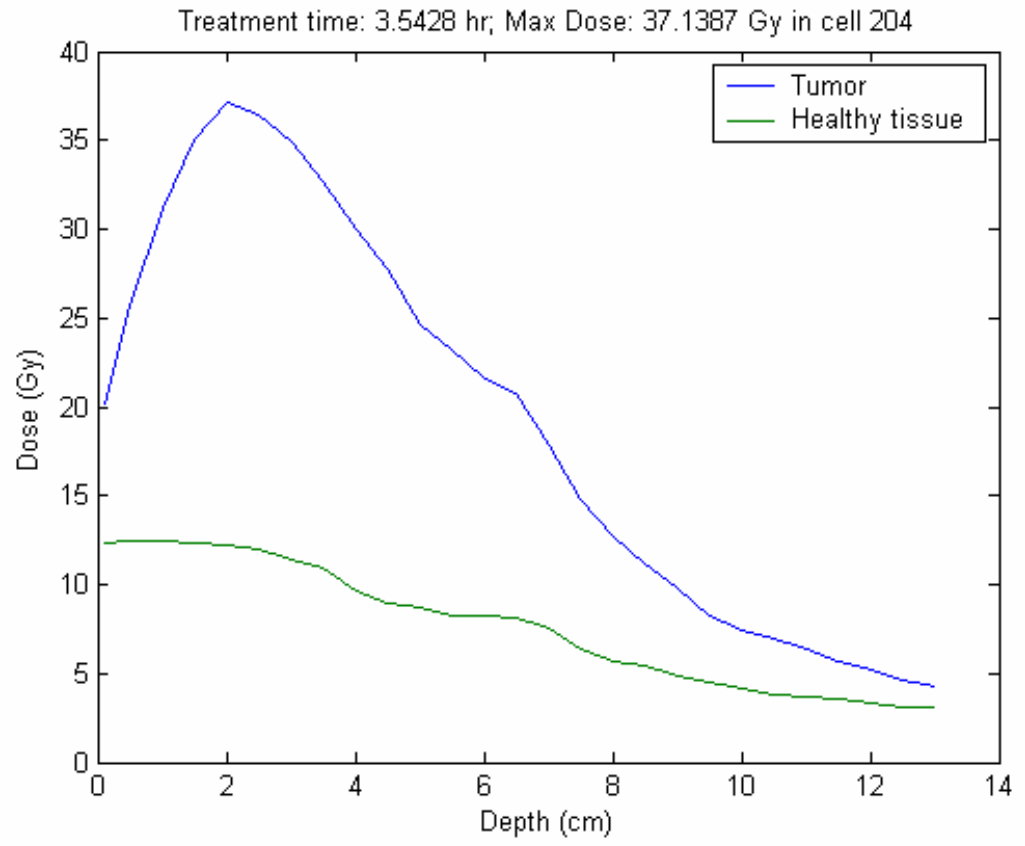

Figure 21: Total dose to the healthy tissue and to the tumor as a function of depth 


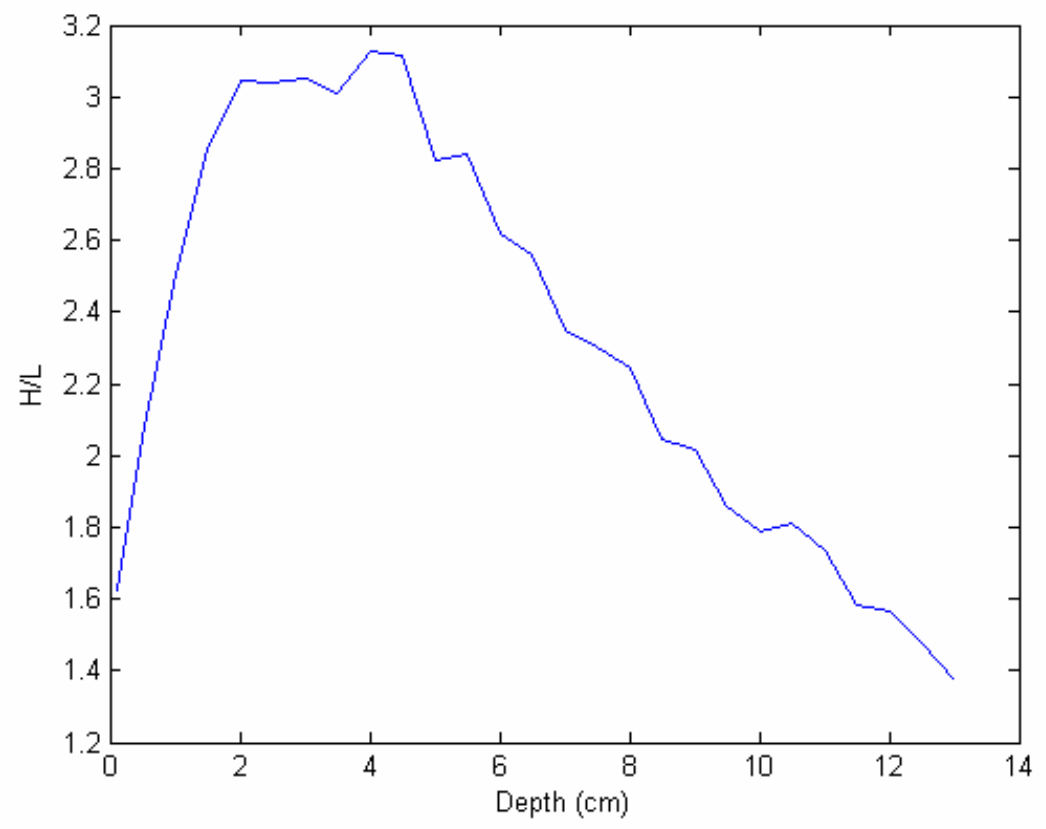

Figure 22: Tumor-to-healthy tissue dose ratio (H/L)

As part of this analysis a study was performed of whether a combination of a relatively thin $\mathrm{BeO}$ layer followed by $\mathrm{Pb}$ on the outer side would offer a better performance than $\mathrm{BeO}$ alone, considering the good reflective properties of $\mathrm{Pb}$, especially for fast neutrons. The resulting performance was found to be inferior to that of $\mathrm{BeO}$ alone: therefore we opted for an "all $\mathrm{BeO}$ " solution as an upper boundary on the system performance.

\subsection{Summary of SCM optimization for $\mathbf{k}_{\text {eff }}$ of $\mathbf{0 . 9 9 2}$}

The SCM and reflector optimizations reported upon in Sections 5.1 through 5.4 under the $\mathrm{k}_{\mathrm{eff}}=0.992$ constraint achieved a significant improvement in the BNCT facility performance going from an irradiation time of more than 24 hours to about 3.4 without sacrificing the lethality range while reducing the cost of needed uranium from $\$ 215,000$ to $\$ 60,300$.

However, it is doubtable that a $\mathrm{k}_{\text {eff }}$ of 0.992 will be licensable as it is subcritical by only slightly more than $1 \$$ of reactivity. A significantly safer design $k_{\text {eff }}$ is 0.98 ; many accelerator driven sub-critical reactors designed for transmutation of nuclear waste use 0.98 as their maximum $\mathrm{k}_{\text {eff }}$ design goal. Unfortunately, this seemingly small reduction in $\mathrm{k}_{\mathrm{eff}}$ translates into a substantial reduction in the SCM neutron multiplication and a comparable increase in the 
required irradiation time. Thus, whereas the reference $\mathrm{k}_{\mathrm{eff}}=0.992$ system has a multiplication $\left(\mathrm{M}=1 /\left(1-\mathrm{k}_{\text {eff }}\right)\right)$ of 125 and requires an irradiation time of 3.34 hours, reducing $\mathrm{k}_{\text {eff }}$ to 0.98 would reduce the multiplication to 50 and increase the irradiation time to about 8.35 hours. Hence further design improvements are needed.

Several other cases where studied in the course of this optimization, specifically:

1 the insertion of a Be layer at the interface between the SCM and the CNS, to take advantage of the $\mathrm{n}, 2 \mathrm{n}$ reaction with the $2.5 \mathrm{MeV}$ neutrons;

2 the insertion of an $\mathrm{H}_{2} \mathrm{O}$ layer at the interface between the SCM and the BSA to increase the fission density towards the end of the SCM;

3 the insertion of a cone ${ }^{4}$ in the back of the SCM, later substituted by the insertion of the entire CNS inside the SCM, fill it with.

Each of these modifications where compared based on the necessary treatment time, but none showed any significant improvement.

\subsection{Reduction of $\mathbf{k}_{\text {eff }}$ from 0.992 to 0.98}

The design change adopted for reducing $\mathrm{k}_{\text {eff }}$ to 0.98 by slightly decreasing the radius of the vertical plates of the SCM shown in Figure 20 from $17.61 \mathrm{~cm}$ to $16.75 \mathrm{~cm}$; everything else is left unchanged. The total cost of the uranium is reduced slightly to $\$ 57,400$.

Figure 23 shows that the maximum tumor dose is about 36 Gy at $2 \mathrm{~cm}$ depth inside the brain, achieved in about 6.3 hours of treatment, which is better than the expected 8.45 hours guessed based on the expected change in the subcritical multiplication, as estimated in the previous section. Considering that the tumor control dose is about $20 \mathrm{~Gy}$, this setup could kill tumors situated up to about $6 \mathrm{~cm}$ depth. Figure 24 shows the ratio between tumor dose $(\mathrm{H})$ and healthy tissue dose (L). H/L peaks at a depth of $3-4 \mathrm{~cm}$ at about 3.2 , and is as low as 1.5 in the skin and in the deepest part of the brain. The dose rate at $2 \mathrm{~cm}$ depth is $5.7 \mathrm{~Gy} /$ hour.

\footnotetext{
${ }^{4}$ The cone has been filled with aluminum and in a second configuration with $5 \%$ enrichment ${ }^{235} \mathrm{U}$ slabs.
} 


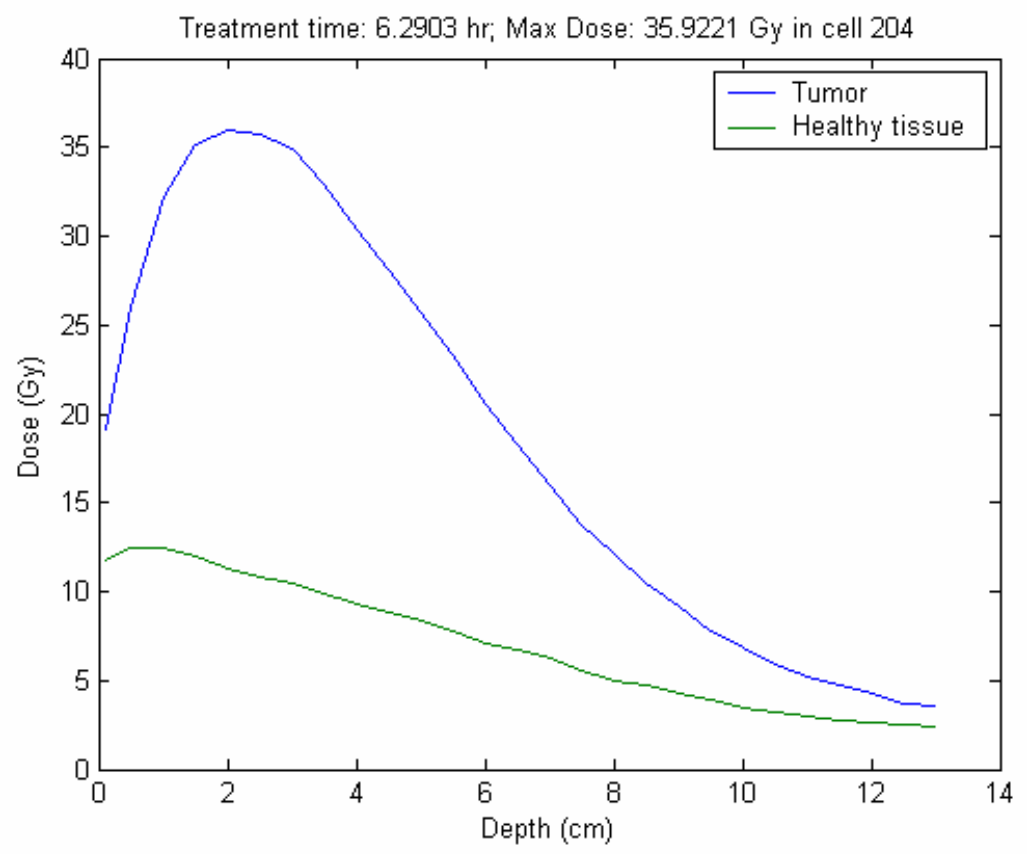

Figure 23: Total dose to the healthy tissue and to the tumor as a function of depth

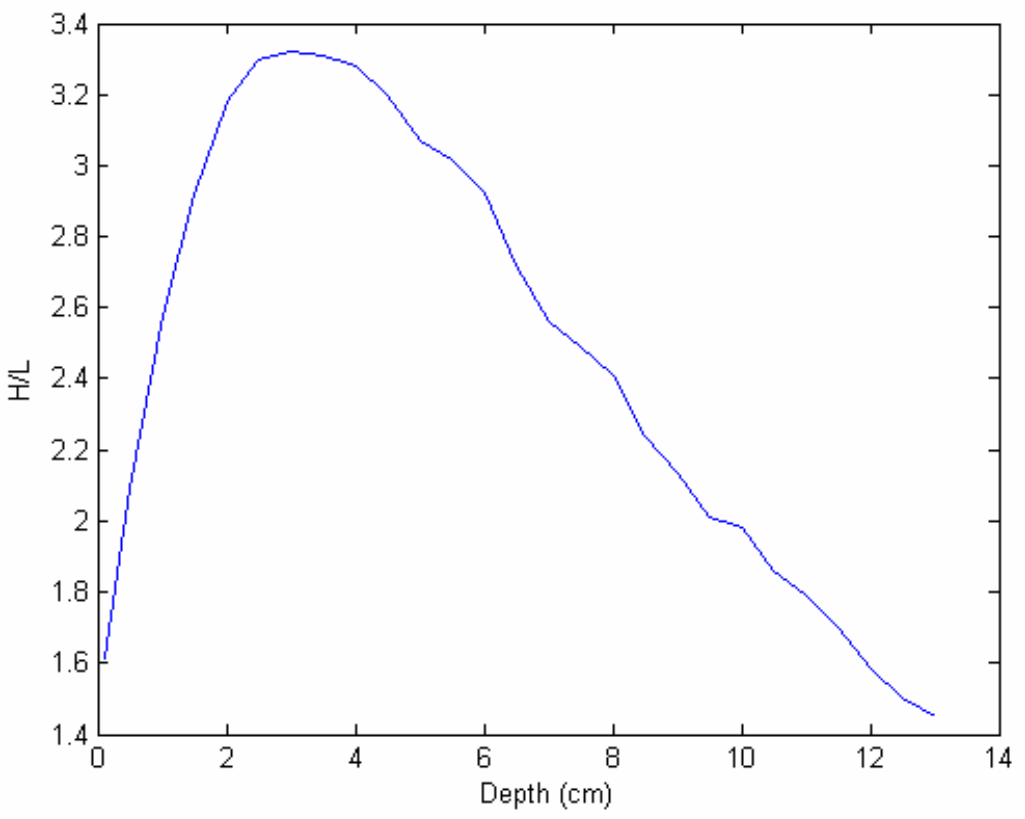

Figure 24: Tumor-to-healthy tissue dose ratio (H/L) 


\section{BSA OPTIMIZATION STUDIES}

The optimization of the beam shaping assembly (BSA) composition is done using the optimal sub-critical multiplier described in section 5.6 and shown in amplification in Figure 25. The SCM is made of two $20 \%$ enriched uranium metal fuel cylinders surrounding the CNS coaxially and four circular elements positioned between the CNS and the BSA. The horizontal cylinders are $20.995 \mathrm{~cm}$ long - exactly as much as the CNS - and $0.06 \mathrm{~cm}$ thick, with $0.05 \mathrm{~cm}$ clad on both sides and $0.84 \mathrm{~cm}$ water between them. The innermost cylinder has a radius of 13.5 $\mathrm{cm}$. Similarly the vertical plates are $0.06 \mathrm{~cm}$ thick and the clad is $0.05 \mathrm{~cm}$ thick. The water gaps in-between the fuel elements are $0.84 \mathrm{~cm}$ thick. The entire SCM is encased in aluminum cladding, which is the physical barrier between the CSN and the SCM. Immediately after the enclosing cladding there is a water layer of $0.84 \mathrm{~cm}$, followed by the 4 uranium plates. The last uranium plate is in direct contact with the BSA.

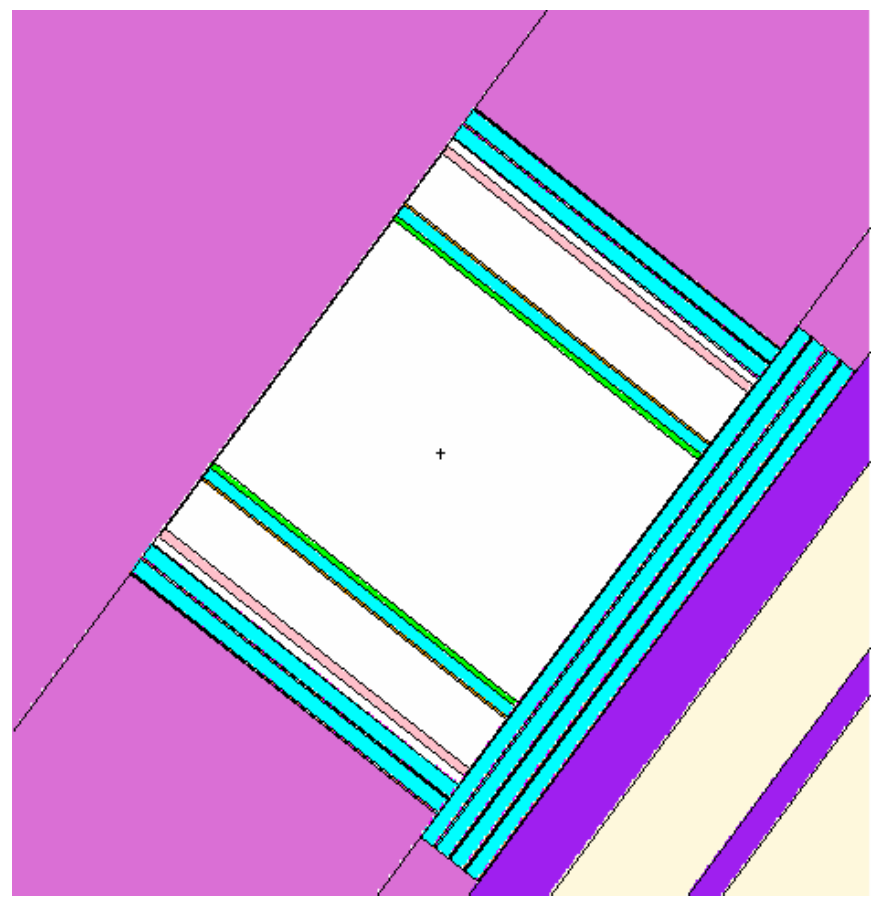

Figure 25: CNS and SCM, $\mathrm{x}-\mathrm{z}$ view. The pink is the beryllium oxide reflector surrounding the CNS (the white central space); the light blue is water between the uranium plates of the SCM (which are too thin to be distinguished in this plot); the violet is the lead in the BSA and the white in the bottom white corner is the ${ }^{7} \mathrm{LiF}$ in the BSA. 
The following sections describe, in chronological sequence, the successive BSA optimization steps. Un-successful design modifications are also reported as they add to our understanding of the BSA performance and the information they provide could be useful for future studies.

\subsection{Case One: Reference design}

The reference BSA is that described in Section 4. Figure 26 shows the contribution to the healthy tissue dose (L) of the gamma-rays, neutron absorption in boron and neutron absorption in nitrogen. The gamma-rays contribution is found to be the dominant. This is undesirable as this component delivers the same dose to the healthy tissue as to the tumor. Fortunately this component can be easily reduced by inserting additional high $\mathrm{Z}$ material in the beam path.

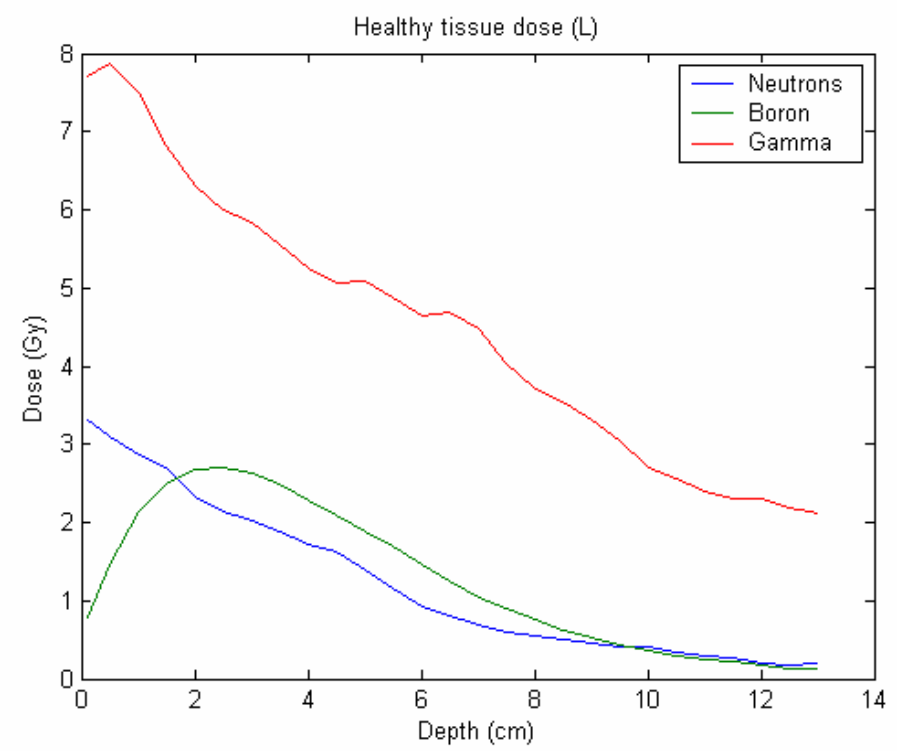

Figure 26: Components of the healthy tissue dose (L); Case 1

Figure 27 shows the normalized neutron current spectrum at various depths in the BSA. The current considered is the net current through planes in the BSA that are perpendicular to the BNCT facility axis. A negative current at the SCM-BSA boundary implies that neutrons of that specific energy stream back from the BSA to the SCM. Previous studies established that neutrons with energy below $1 \mathrm{KeV}$ and above $20 \mathrm{KeV}$ are not desirable as their tumor dose to normal tissue dose contribution ratio is too low [1]. Observing the current spectrum between the lead layer and the brain it appears that the spectrum is too soft: the components below $1 \mathrm{keV}$ are 
therapeutically undesirable. Therefore some changes need to be made to avoid solwing down the neutrons excessively.

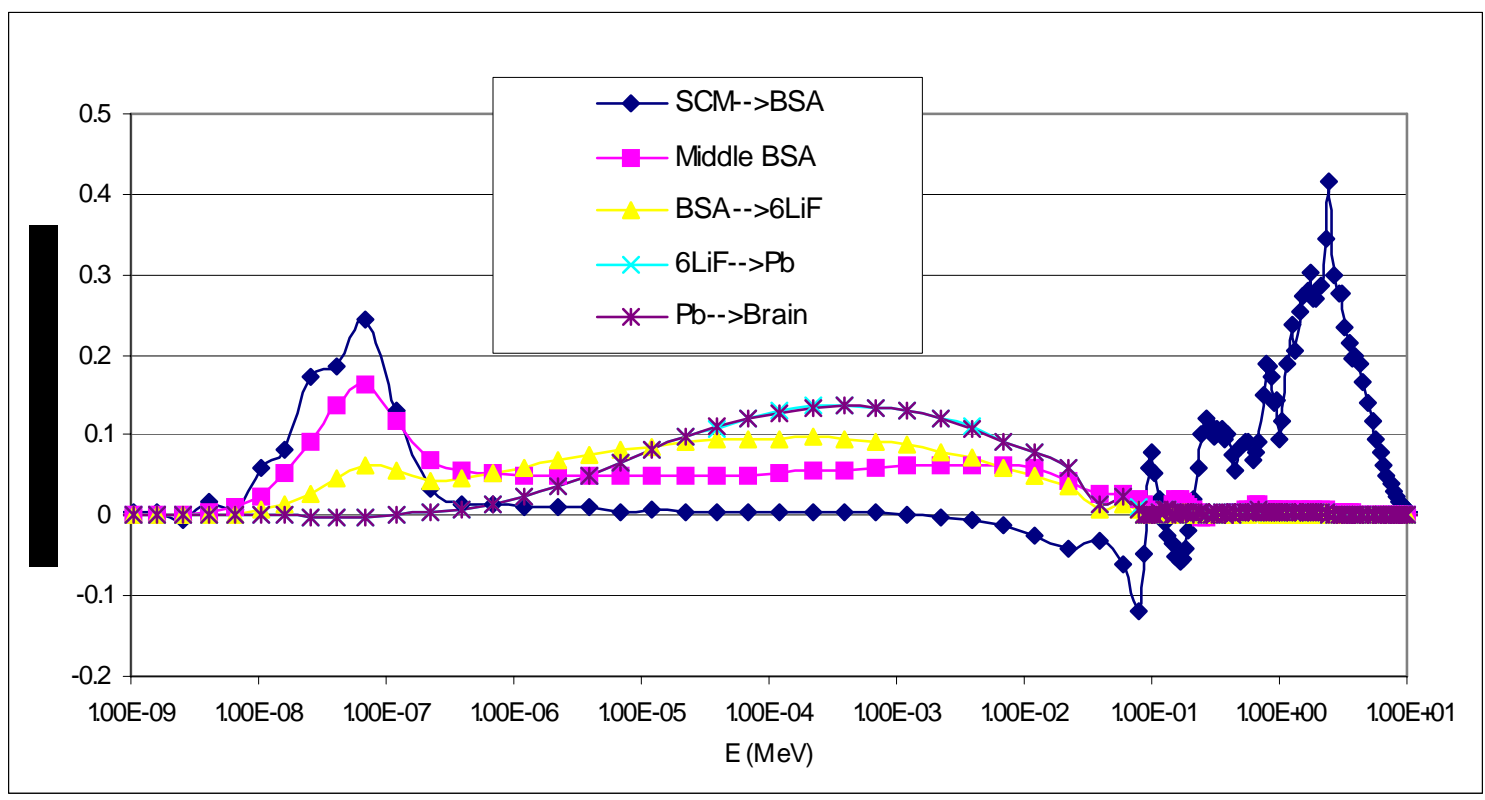

Figure 27: Net neutron currents per unit lethargy at various depths inside the BSA

\subsection{Case Two: SWAN-optimized BSA}

In a previous study $[13,14]$ the one-dimensional optimization code SWAN was set to find the highest possible dose to the tumor, $\mathrm{H}$, for a given $\mathrm{H} / \mathrm{L}$ - tumor-to-healthy tissue dose ratio which was selected for this study to be somewhat arbitrarily fixed at 3.3. The BSA was driven by a spherical annulus-shaped sub-critical multiplier made of $20 \%$ enriched uranium and designed to have a $\mathrm{k}_{\text {eff }}$ of 0.98 . A number of materials and BSA length where considered. The optimal BSA was found to be $51 \mathrm{~cm}$ long made of only lead and ${ }^{7} \mathrm{LiF}$, with the exception of a $1 \mathrm{~mm}$ slab of ${ }^{6} \mathrm{LiF}$ at the end towards the patient, mixed in different fractions along the BSA as shown in Figure 28. Since it would be impractical to uniformly mix ${ }^{7} \mathrm{LiF}$ and lead, the composition of Figure 28 could be approximated by distinct layers of the two materials as shown in Figure 29.

The BSA composition of Figure 29 was used instead of the reference considered in the previous section. Additionally the alumina reflector of the BSA has been replaced by lead, because of its better reflective properties and its effectiveness in stopping the un-wanted gamma 
rays. A cross sectional view of the resulting system is plotted in Figure 30. The thickness of the various layers in the SWAN-optimized BSA are shown in Table IV.

Table IV: Dimensions of the SWAN-optimized BSA, as shown in Figure 30.

\begin{tabular}{|l|r|l|}
\hline \multicolumn{2}{|l|}{ Thickness of } & \\
\hline $\mathrm{Pb}$ & 3.5 & $\mathrm{~cm}$ \\
\hline${ }^{7} \mathrm{LiF}$ & 6 & $\mathrm{~cm}$ \\
\hline $\mathrm{Pb}$ & 1.5 & $\mathrm{~cm}$ \\
\hline${ }^{7} \mathrm{LiF}$ & 21 & $\mathrm{~cm}$ \\
\hline $\mathrm{Pb}$ & 1 & $\mathrm{~cm}$ \\
\hline${ }^{7} \mathrm{LiF}$ & 8 & $\mathrm{~cm}$ \\
\hline $\mathrm{Pb}$ & 1 & $\mathrm{~cm}$ \\
\hline${ }^{7} \mathrm{LiF}$ & 8 & $\mathrm{~cm}$ \\
\hline $\mathrm{Pb}$ & 0.9 & $\mathrm{~cm}$ \\
\hline${ }^{6} \mathrm{LiF}$ & 0.1 & $\mathrm{~cm}$ \\
\hline
\end{tabular}

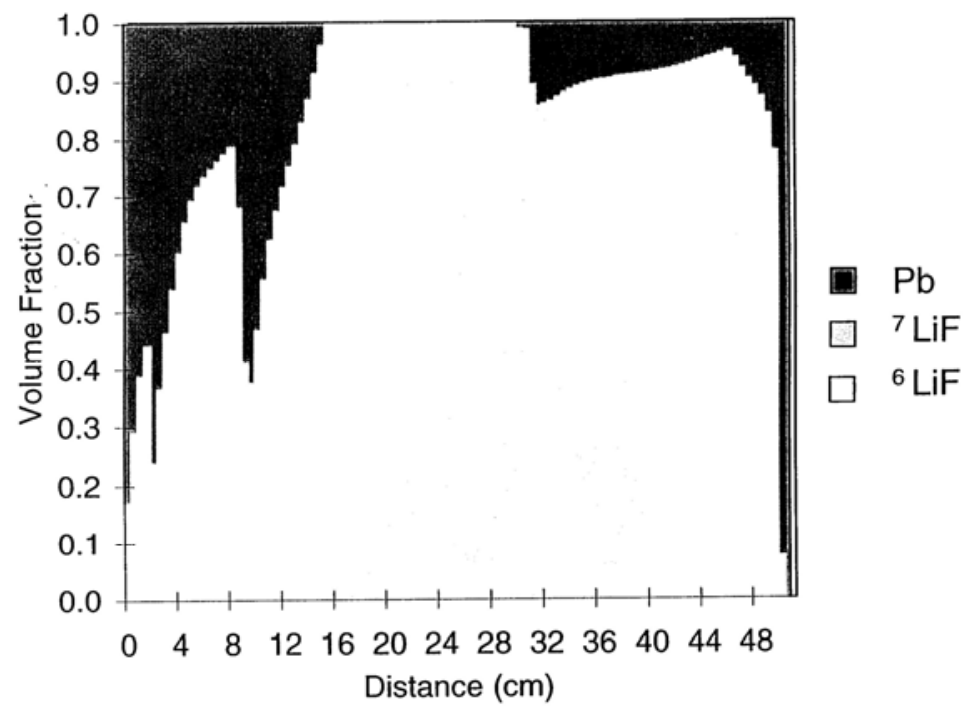

Figure 28: Optimal BSA configuration found by SWAN. The ${ }^{6} \mathrm{LiF}$ is not visible, being a thin layer at the right-hand side of the assembly 


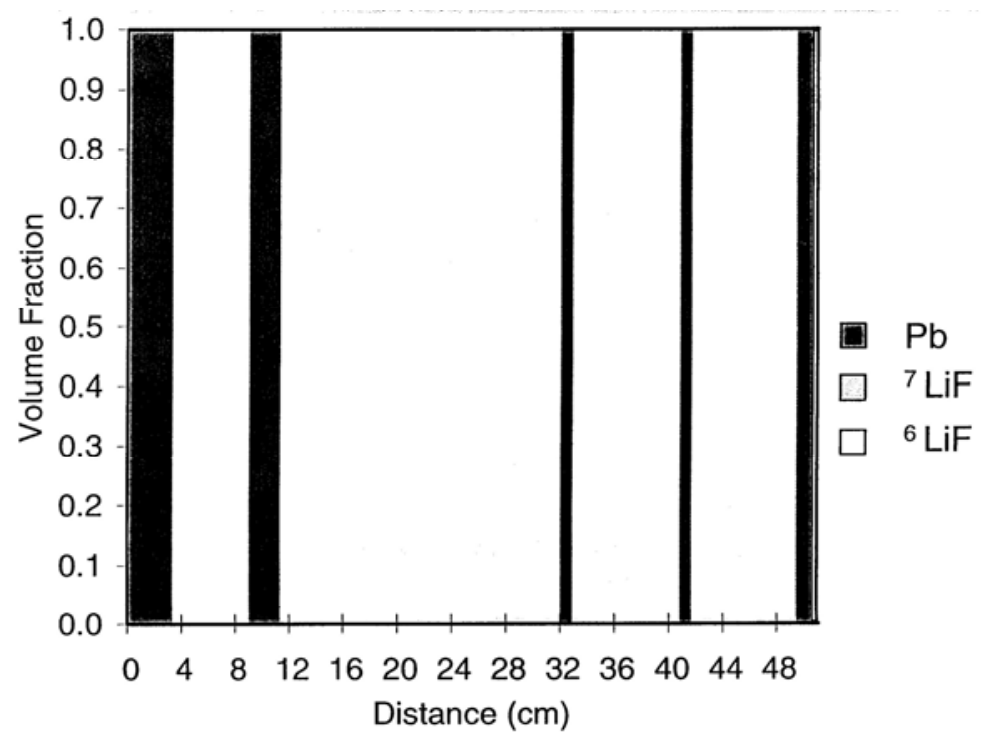

Figure 29: Approximation of the SWAN solution that is made of slabs of $\mathrm{Pb}$ and ${ }^{7} \mathrm{LiF}$. The ${ }^{6} \mathrm{LiF}$ is a thin layer at the right-hand side of the assembly

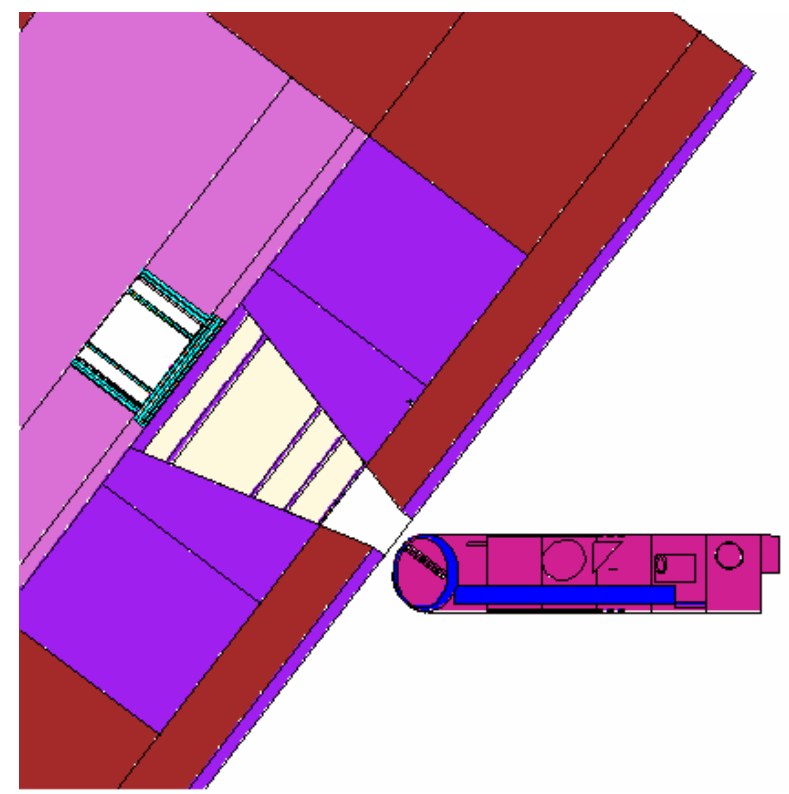

Figure 30: Cross sectional view of the BSA optimized by SWAN as it is inserted into the system; Case 2.

The results of this simulation are presented in Figure 31 to Figure 33. The maximum dose is about $43 \mathrm{~Gy}$ at about $2 \mathrm{~cm}$ depth, delivered in 7.5 hours of treatment, with a dose rate of 5.73 
Gy/hours. The H/L reaches a maximum of about 4.3 at $4 \mathrm{~cm}$ depth and drops no lower than 2.5 as deep as $12 \mathrm{~cm}$ inside the head. The tumor control dose of $20 \mathrm{~Gy}$ is reached in the first $6.5 \mathrm{~cm}$.

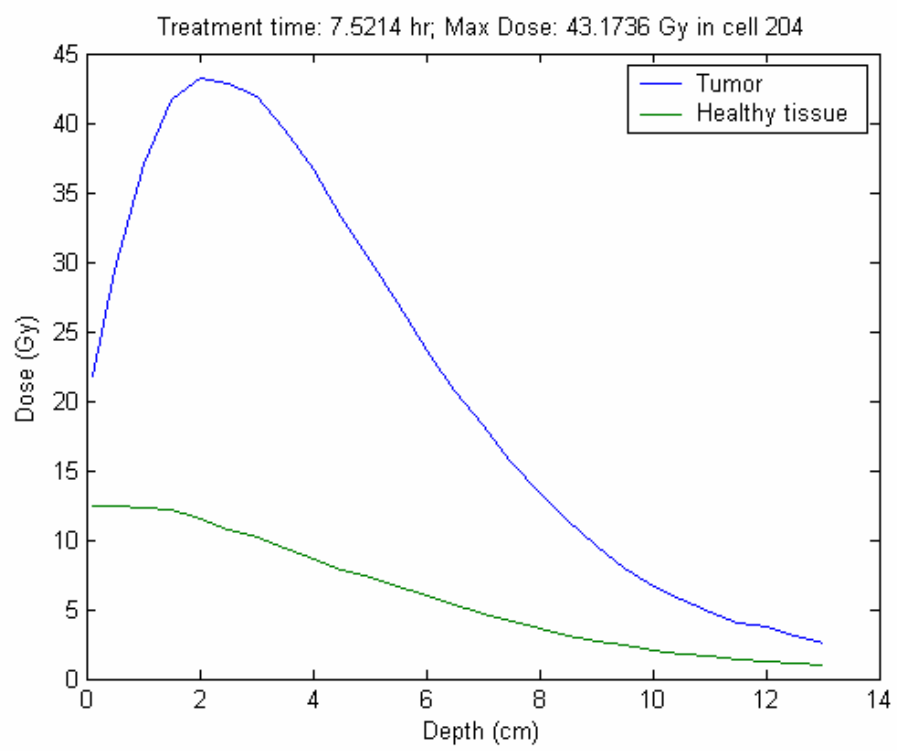

Figure 31: Total dose to the healthy tissue and to the tumor as a function of depth; Case 2

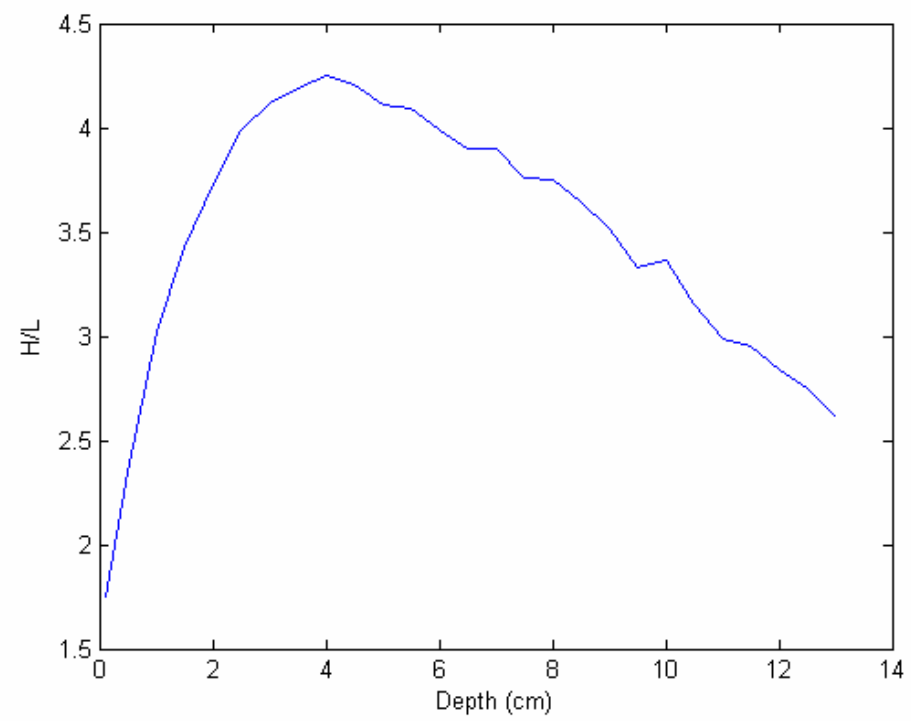

Figure 32: Tumor-to-healthy tissue dose ratio $(\mathrm{H} / \mathrm{L})$; Case 2

Figure 33 shows the components of the healthy tissue dose. The gamma component is much lower than in the reference case, due to extra amount of lead added in the BSA and in the 
reflector - compare Figure 33 and Figure 26. However, the neutron component, excluding through capture in boron, is too large.

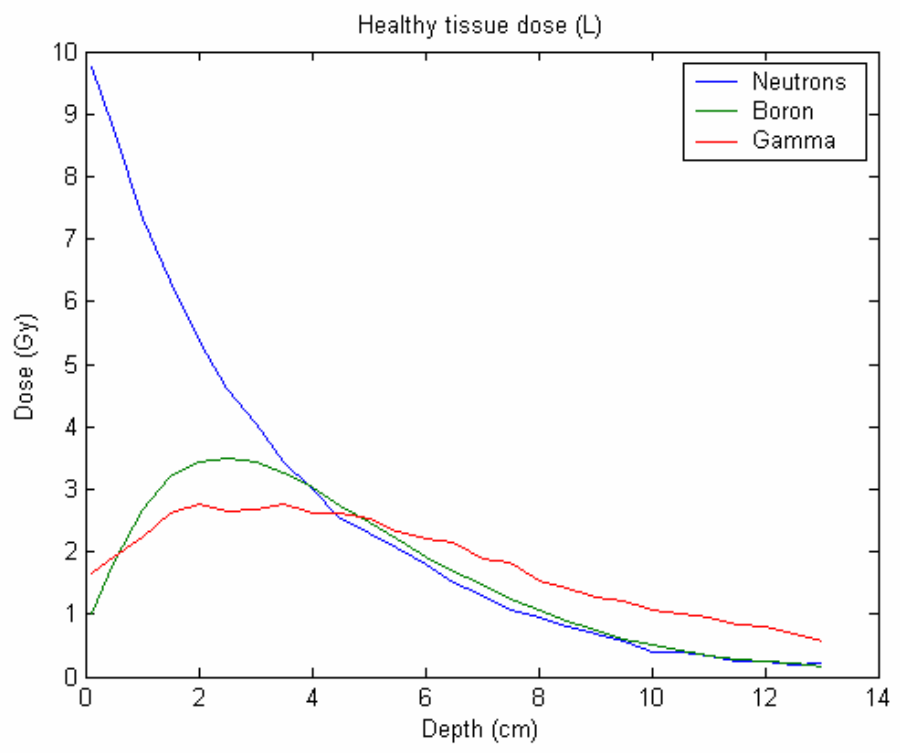

Figure 33: Components of the healthy tissue dose (L); Case 2

\subsection{Case three: $1 \%{ }^{6} \mathrm{LiF}$ added to the last 2 zones}

The BSA is of an identical configuration as in the previous case but the ${ }^{7} \mathrm{LiF}$ in the last two zones was "doped" with $1 \%{ }^{6} \mathrm{LiF}$ with the purpose of cutting down the thermal neutron component. A cross sectional view of the resulting system is shown in Figure 34.

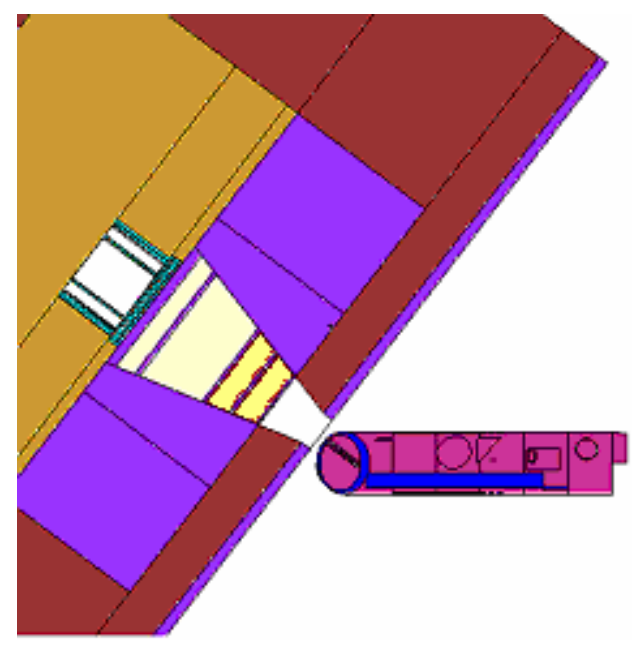

Figure 34: Cross sectional view of Case 3 system: $1 \%{ }^{6} \mathrm{LiF}$ added to the last two BSA zones 
The results for this system are presented in Figure 35 to Figure 37. The maximum dose drops to 22.22 Gy at a depth of about $2.5-3 \mathrm{~cm}$, reached with an irradiation time of about 6.5 hours: the dose rate is about $3.4 \mathrm{~Gy} /$ hour. The neutron contribution to the healthy dose $\mathrm{L}$ shown in Figure 37 is not better than in Case 2 of Figure 33. This is an indirect confirmation that the high dose to the skin is due to the fast component of the neutrons spectrum.

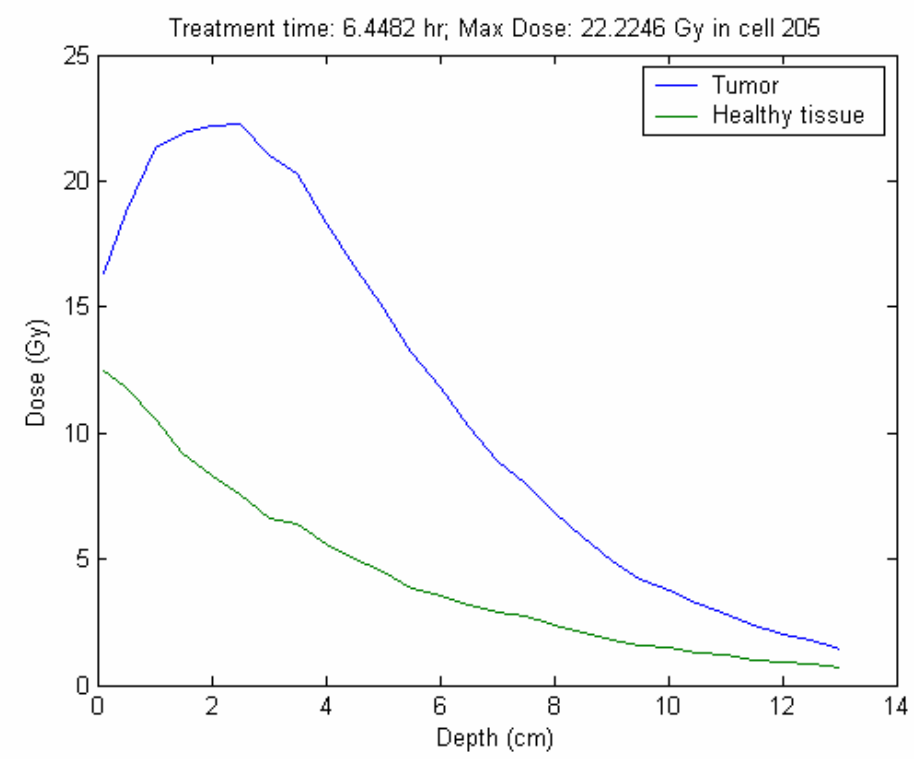

Figure 35: Total dose to the healthy tissue and to the tumor as a function of depth; Case 3

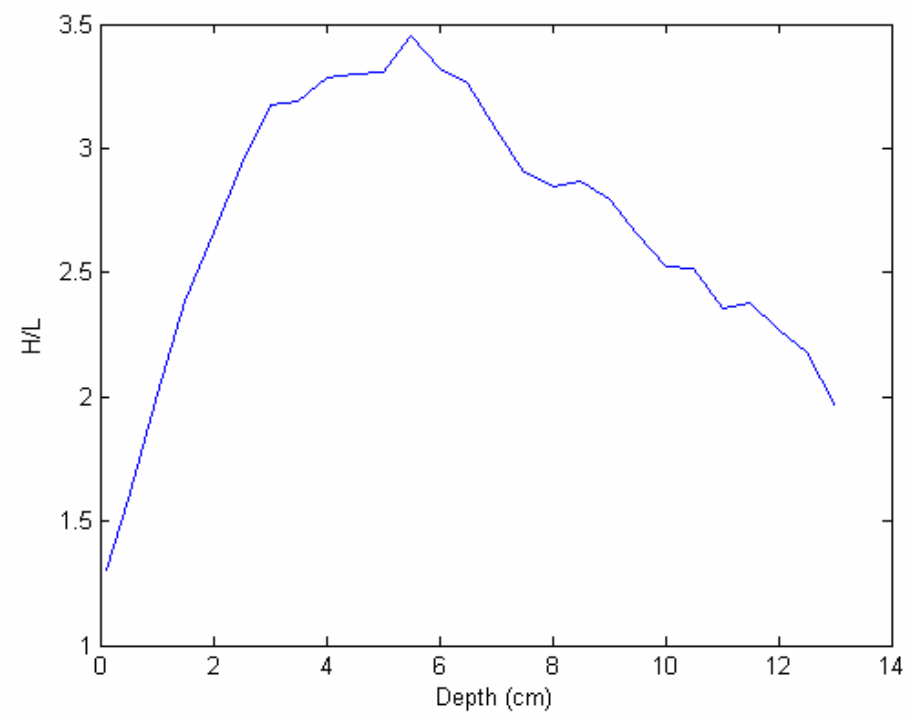

Figure 36: Tumor-to-healthy tissue dose ratio $(\mathrm{H} / \mathrm{L})$; Case 3 


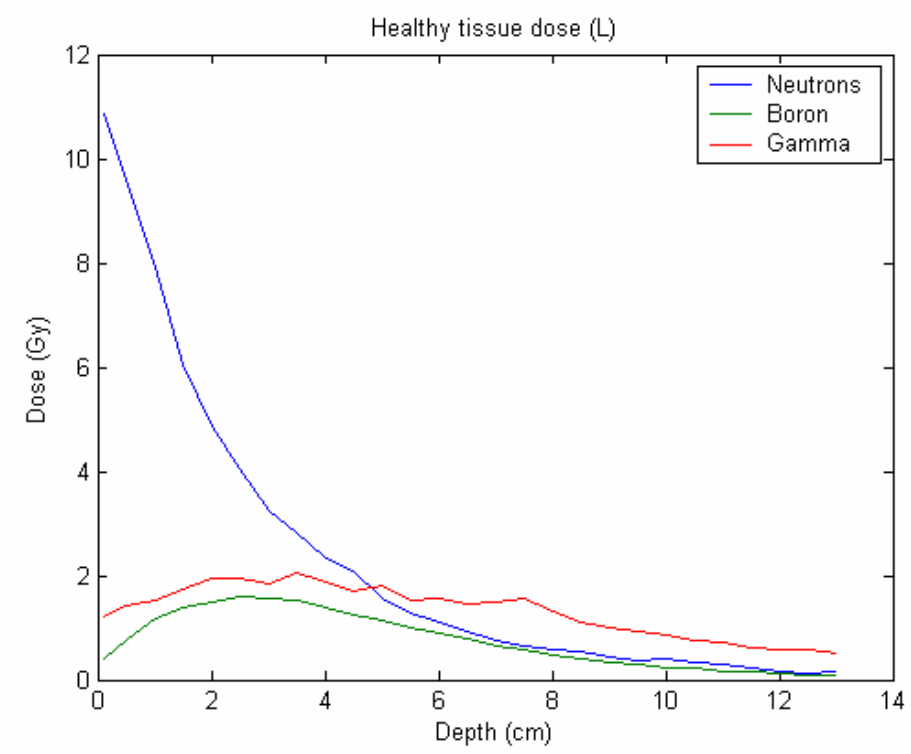

Figure 37: Components of the healthy tissue dose (L); Case 3

\subsection{Case Four: SWAN BSA for D-T $14 \mathrm{MeV}$ neutrons}

The configuration of Case Four is the same as Case Two, but the BSA design is an approximation of an optimized solution found by SWAN [15] for $14 \mathrm{MeV}$ D-T neutrons rather than for $2.45 \mathrm{MeV}$ D-D neutrons. The rationale for considering this BSA is that a fission neutron can be born with up to $10 \mathrm{MeV}$ - significantly higher energy than the D-D neutrons energy. A BSA optimized for $14 \mathrm{MeV}$ neutrons should be more effective in slowing down the fast neutrons; the previous case indicated that the fast neutrons are primarily responsible for the relatively high skin dose.

Figure 387 shows a cross sectional view of the system considered. The $51 \mathrm{~cm}$ long BSA is composed of the following layers: $6 \mathrm{~cm}$ of lead (red in the figure), $22 \mathrm{~cm}$ of iron (pink), $2 \mathrm{~cm}$ of lead, $20 \mathrm{~cm}$ of ${ }^{7} \mathrm{LiF}$ with $1 \%{ }^{6} \mathrm{LiF}$ (yellow), $0.9 \mathrm{~cm}$ of lead and finally $1 \mathrm{~cm}$ of ${ }^{6} \mathrm{LiF}$. 


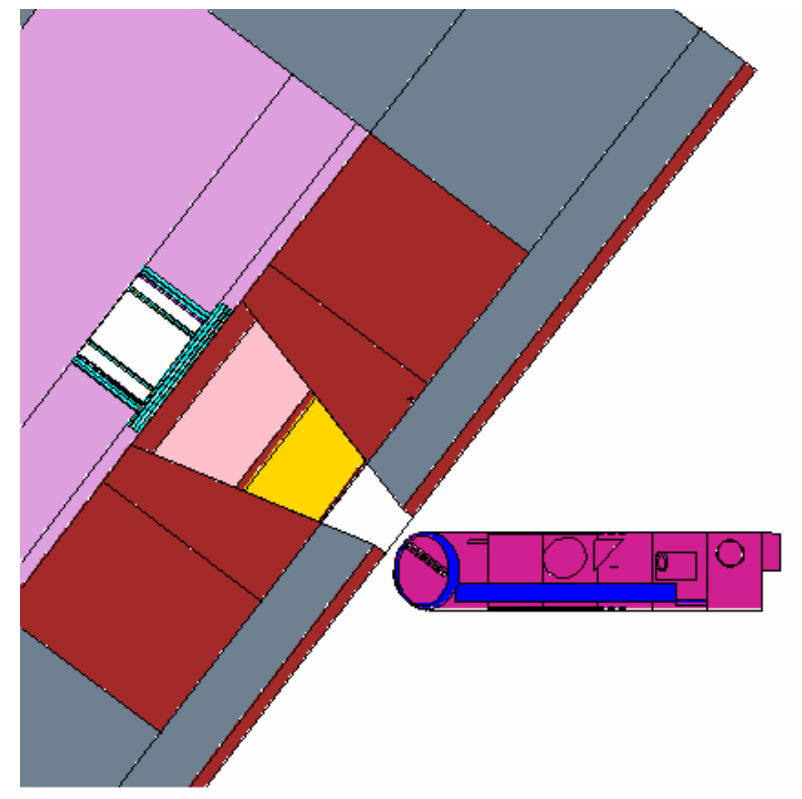

Figure 38: Cross sectional view of the system in Case 4

The results of this simulation are presented in Figure 39 to Figure 41. Figure 39 shows that the maximum allowable dose to the healthy tissue $(12.5 \mathrm{~Gy})$ is reached before the tumor control dose of $20 \mathrm{~Gy}$ is reached anywhere. Therefore this configuration of the BSA will not give any therapeutical benefit. The biggest contribution to the healthy dose (L) comes from the neutron component, which in this case is even bigger than the dose to the tumor due the boron in the shallow part of the brain (see Figure 41). Due to poor performance this case was not pursued. 


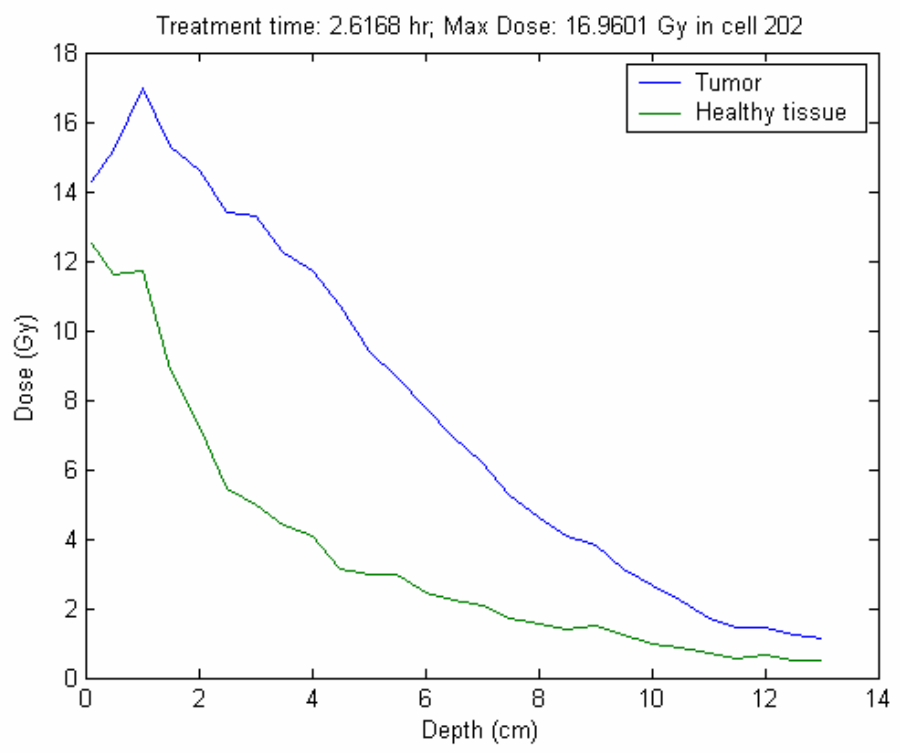

Figure 39: Total dose to the healthy tissue and to the tumor as a function of depth; Case 4

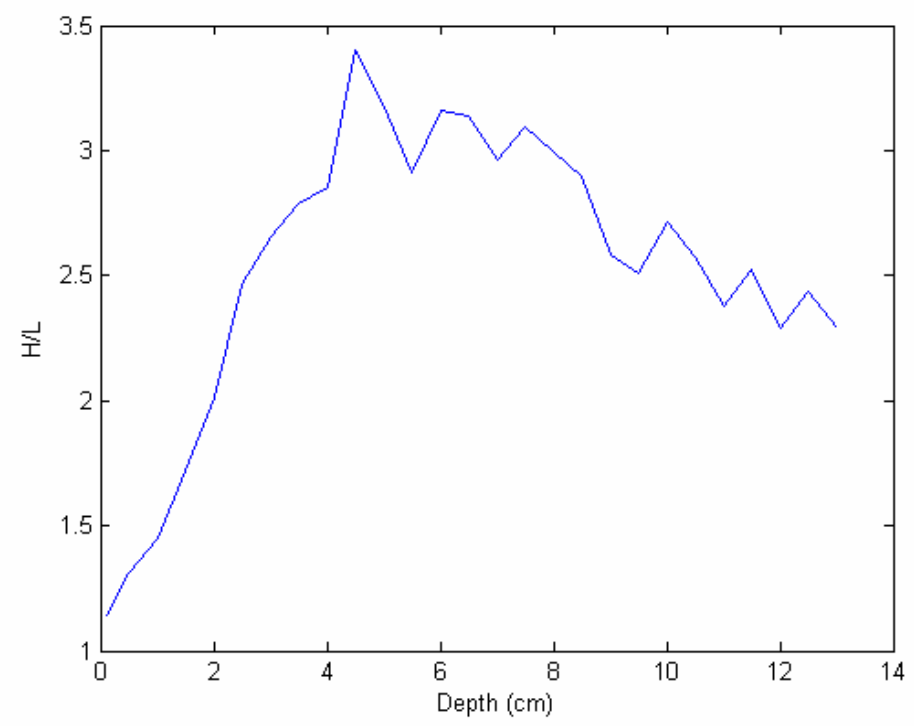

Figure 40: Tumor-to-healthy tissue dose ratio (H/L); Case 4 


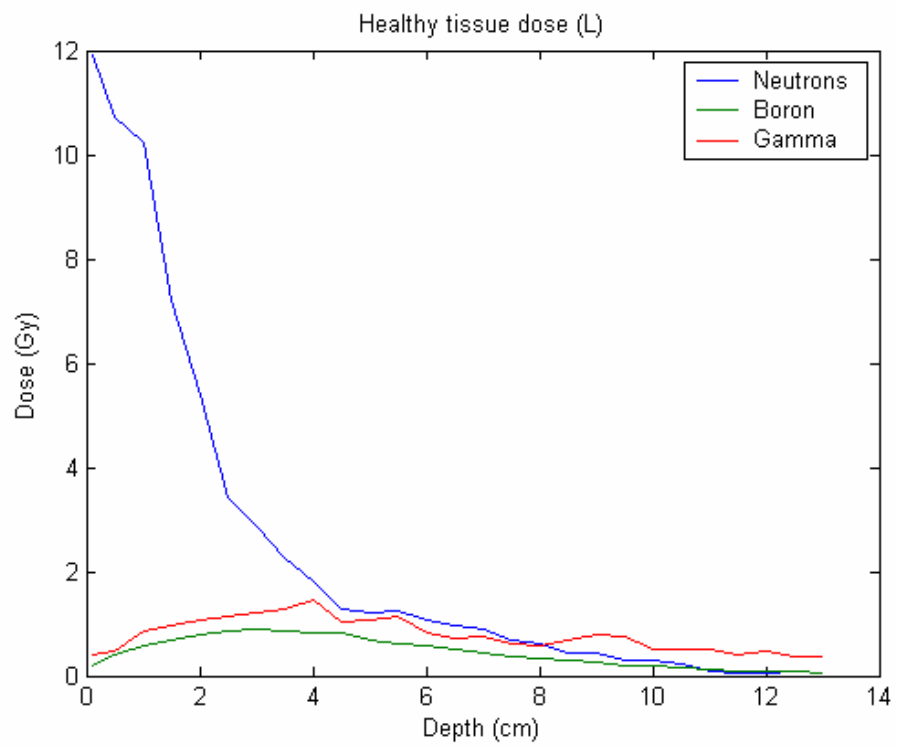

Figure 41: Components of the healthy tissue dose (L); Case 4

\subsection{Case Five: Like Case 2 using Fluental in the last two zones}

This case has the same configuration as case two, but Fluental ${ }^{5}$ replaces ${ }^{7} \mathrm{LiF}$ in the last two zones of the BSA to try to cut down the fast neutron component, which in case 2 gave the highest contribution to the dose to the healthy tissue in the skin and the skull: a cross sectional view of this system is shown in Figure 42. Fluental is chosen because, as shown in Figure 43, the aluminum contained in this material features relatively high scattering cross section above $\sim 30$ $\mathrm{KeV}$; it is higher than that of ${ }^{7} \mathrm{Li}$ and higher than the cross section below $\sim 30 \mathrm{KeV}$.

The results of this simulation are plotted in Figure 44 to Figure 46. The maximum dose of $40.7 \mathrm{~Gy}$ is reached in an irradiation time of about 5 hours at $2 \mathrm{~cm}$ depth: the peak dose rate is about 8.14 Gy/hour. However, Figure 46 shows that the fast neutron component is still responsible for most of the skin dose.

\footnotetext{
${ }^{5}$ Fluental: $\mathrm{Al} / \mathrm{AlF}_{3}(40 \% / 60 \%)$
} 


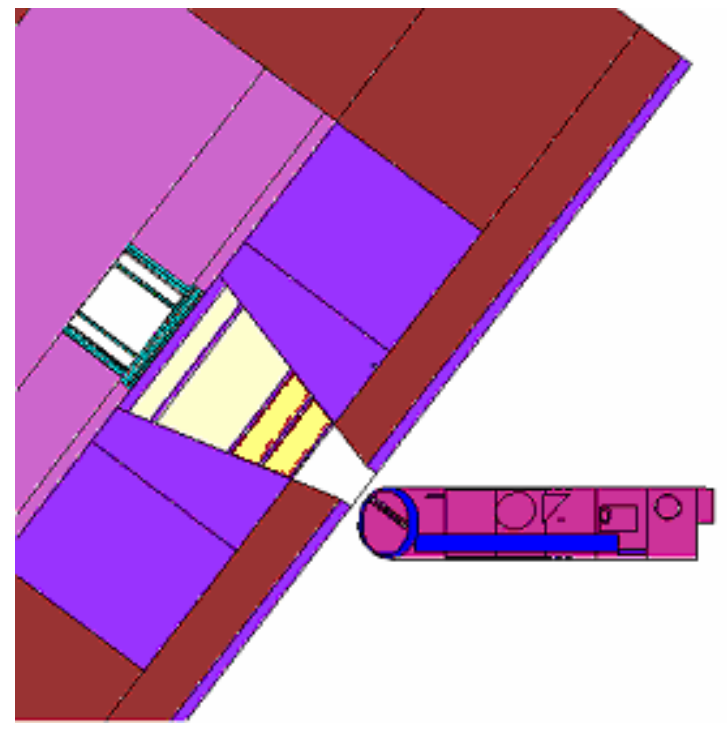

Figure 42: Cross sectional view of the system in Case 5

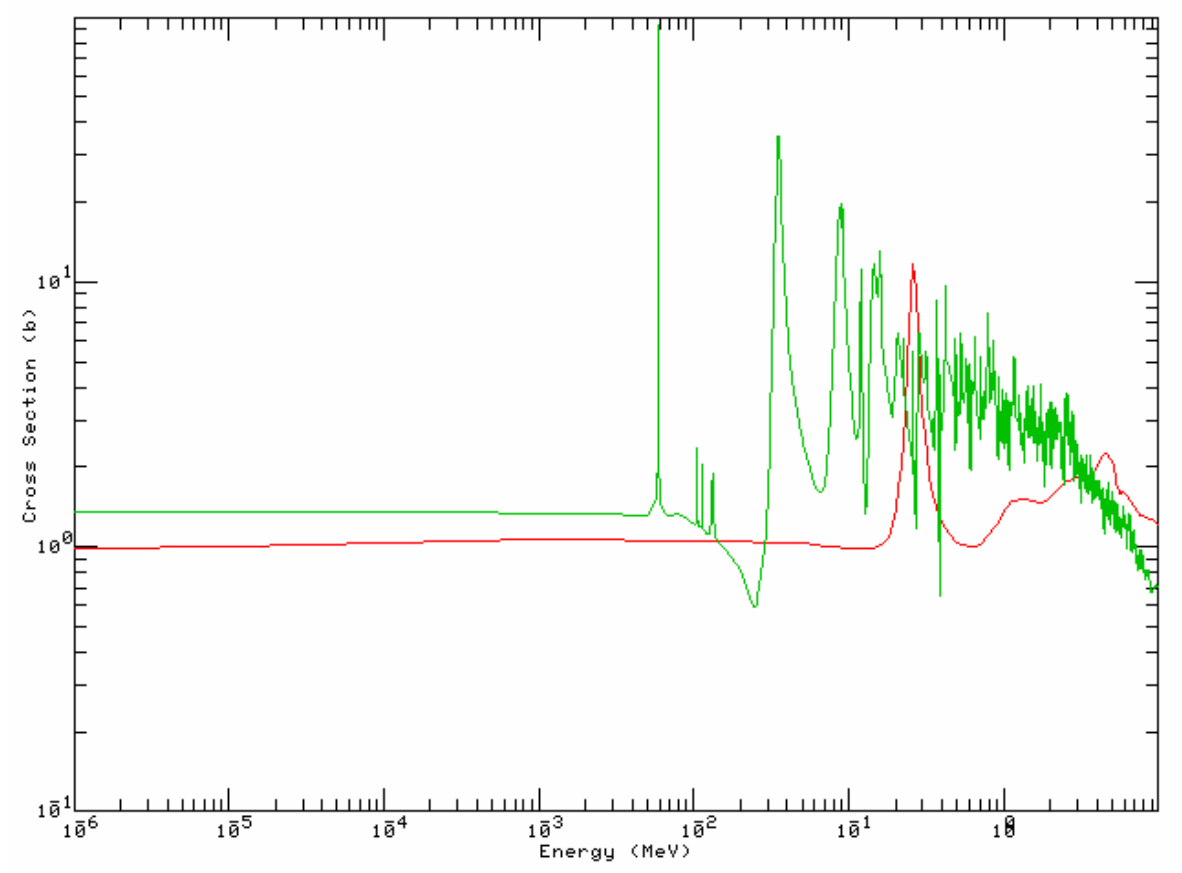

Figure 43: Epithermal and fast scattering cross sections of alluminum (green) and of ${ }^{7} \mathrm{Li}$ (red). 


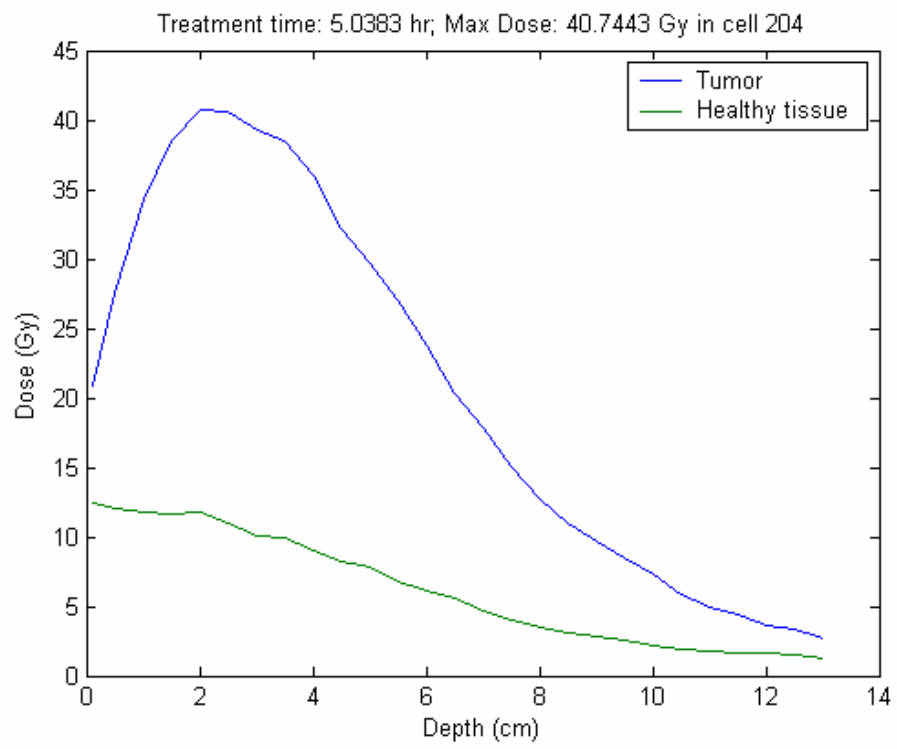

Figure 44: Total dose to the healthy tissue and to the tumor as a function of depth; Case 5

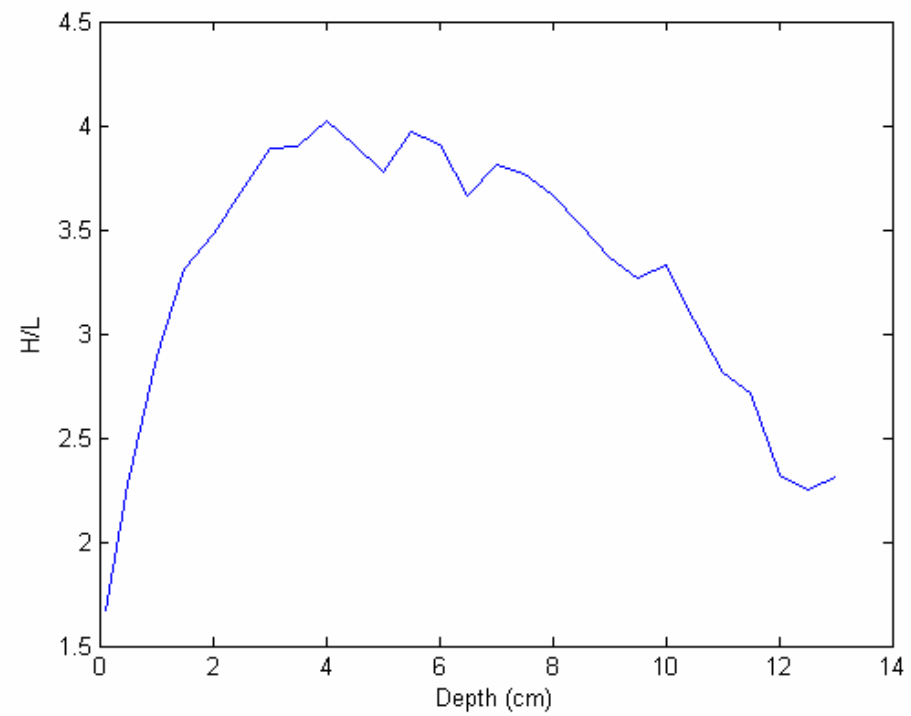

Figure 45: Tumor-to-healthy tissue dose ratio (H/L); Case 5 


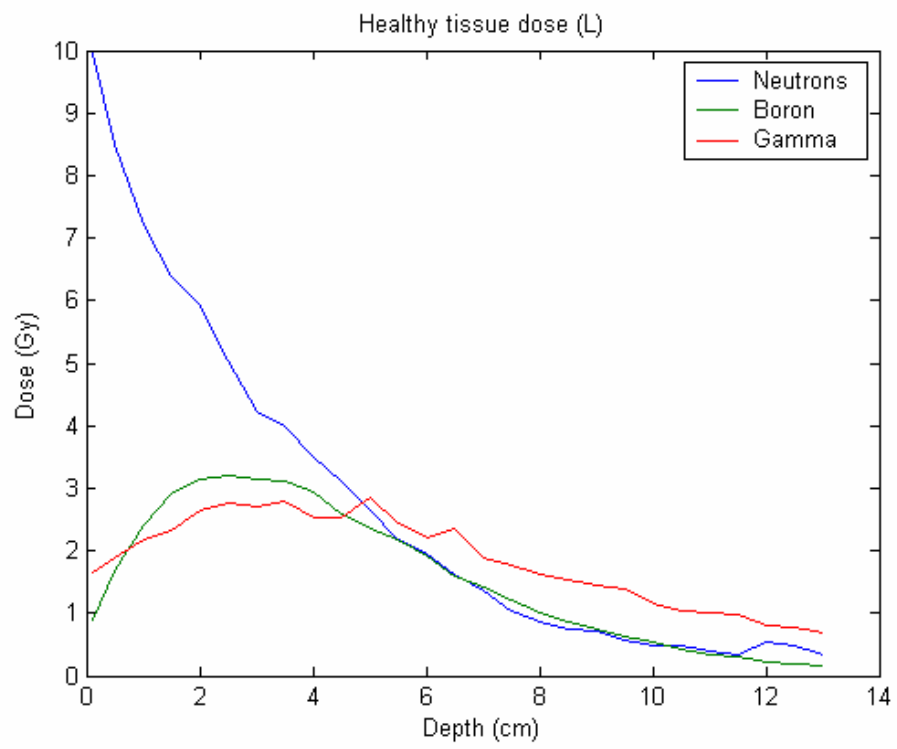

Figure 46: Components of the healthy tissue dose (L); Case 5

\subsection{Case Six: Concentrating $\mathrm{Pb}$ in the last region}

The purpose of this case is to investigate if and to what extent the particular $\mathrm{Pb}$ distribution in the BSA calculated by SWAN is relevant for the superior performance of the SWAN-optimized design. This investigation is done by making the thickness of the last $\mathrm{Pb}$ layer equal the sum of the three lead layers in the BSA of Case 2. Figure 47 shows a cross sectional view of this system. 


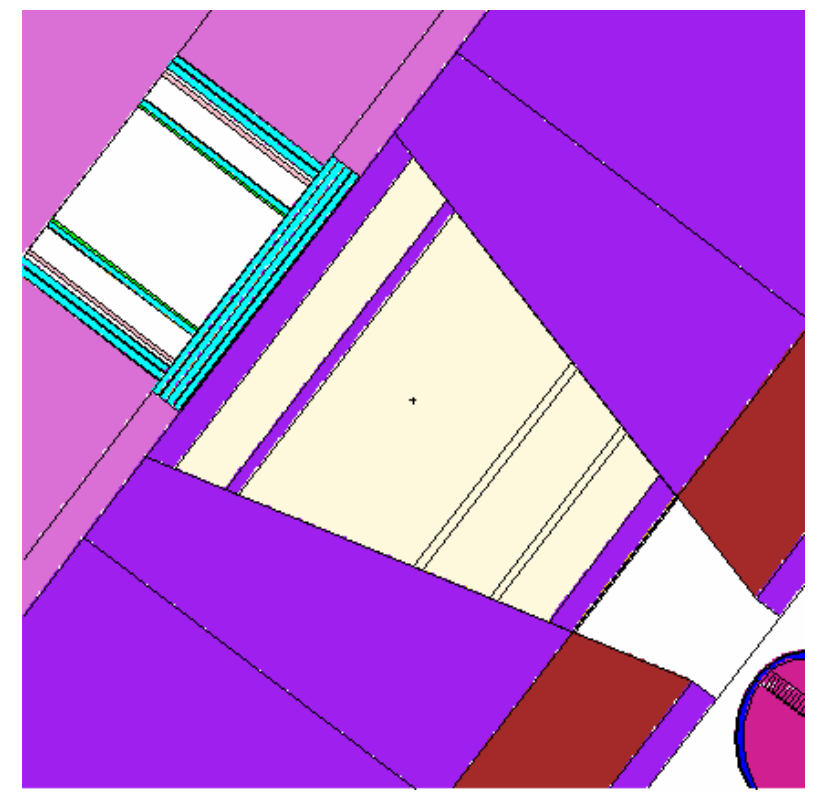

Figure 47: Cross sectional view of the system in Case 6

The results of this simulation are presented in Figure 48 to Figure 50. The maximum dose of about 44.2 Gy at $3 \mathrm{~cm}$ depth is delivered with an irradiation time of about 8 hours. The dose rate is $5.5 \mathrm{~Gy} /$ hour. The overall performance of this system is similar to that of the reference design of SWAN (Case 2), so it is concluded that the order of the Pb slabs in the central and end part of the BSA does not appear to be important. The Pb near the SCM is expected to contribute to the slowing down of the energetic neutrons via inelastic and $(n, 2 n)$ reactions. 


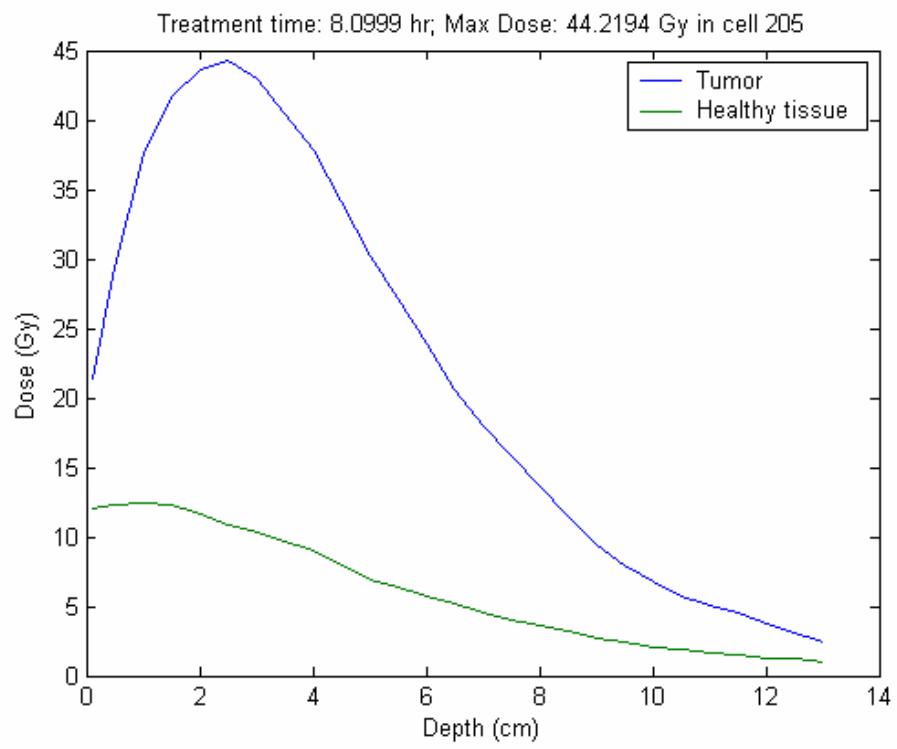

Figure 48: Total dose to the healthy tissue and to the tumor as a function of depth; Case 6

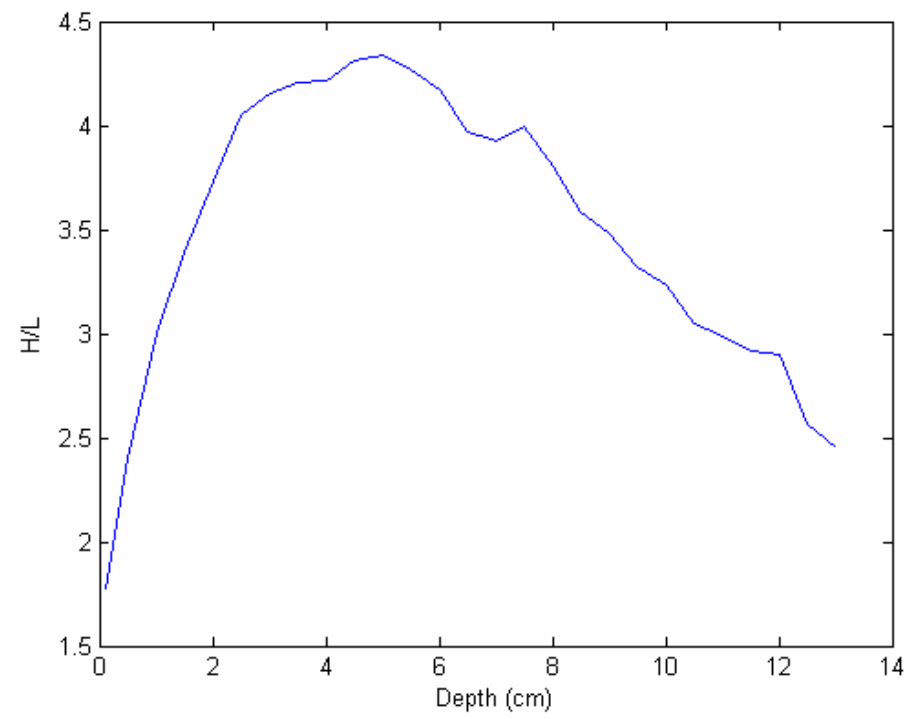

Figure 49: Tumor-to-healthy tissue dose ratio (H/L); Case 6 


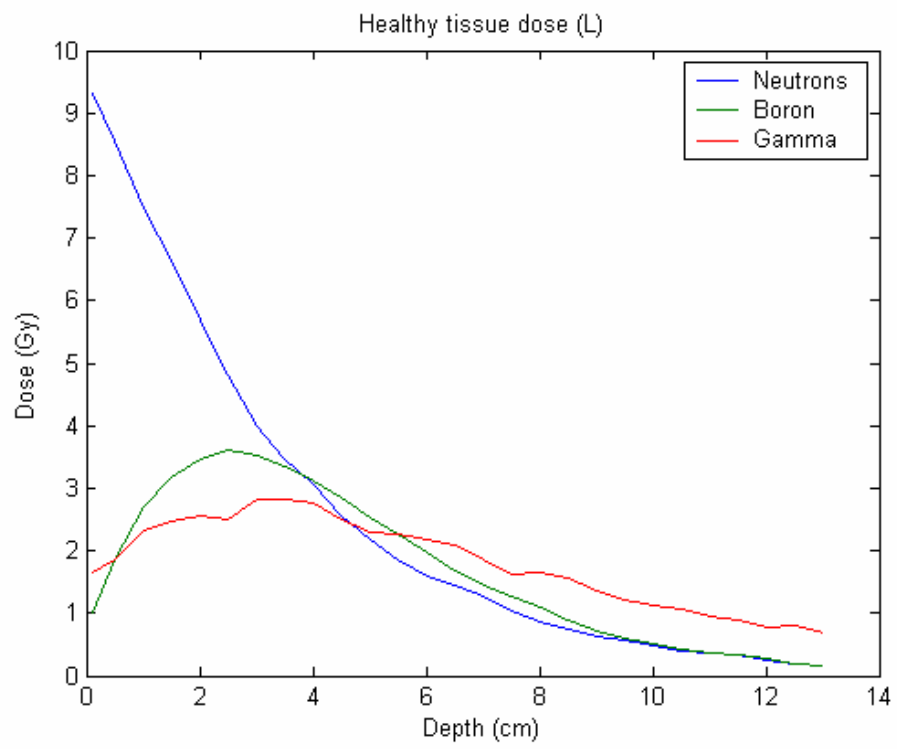

Figure 50: Components of the healthy tissue dose (L); Case 6

\subsection{Case Seven: Lead is eliminated from the last three layers}

The purpose of this case is to investigate whether the substantial amount of lead at the end of the BSA as designed by SWAN is useful. This investigation was done by replacing all the lead from the three layers beyond the center of the BSA by ${ }^{7} \mathrm{LiF}$. Everything else is identical to the configuration of Case 2 (reference SWAN design). Figure 51 shows a cross sectional view of the resulting system. 


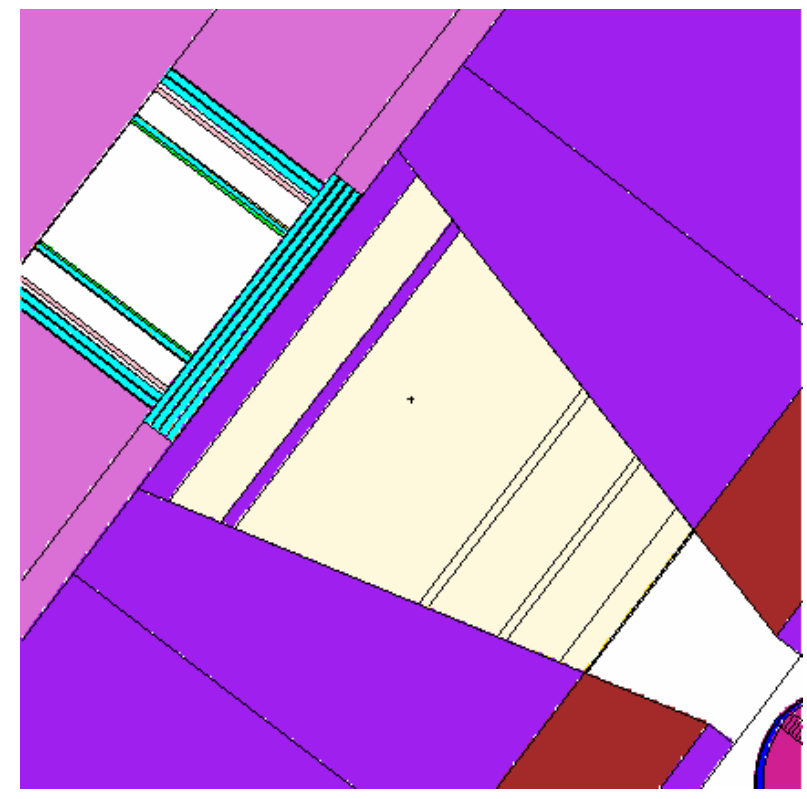

Figure 51: Cross sectional view of the system in Case 7

The results of this simulation are presented in Figure 52 to Figure 54. The maximum dose of $46 \mathrm{~Gy}$, at about $2.5 \mathrm{~cm}$ inside the brain, is reached after an irradiation of about 7.8 hours. The dose rate is about 5.9 Gy/hour. The tumor control dose of 20 Gy is reached in the first $6.5 \mathrm{~cm}$ depth.

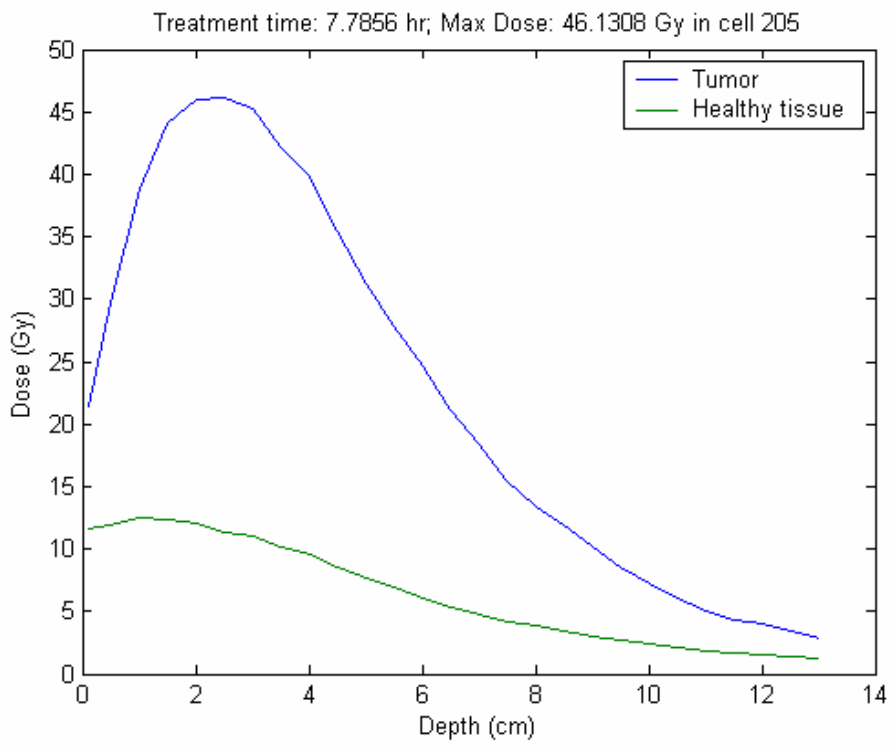

Figure 52: Total dose to the healthy tissue and to the tumor as a function of depth; Case 7 


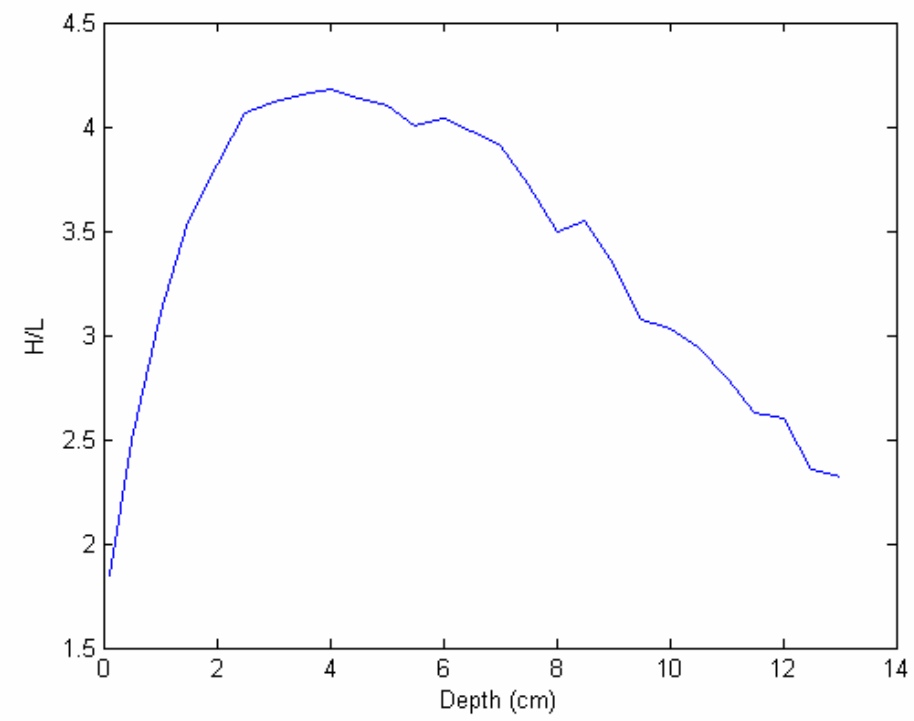

Figure 53: Tumor-to-healthy tissue dose ratio (H/L); Case 7

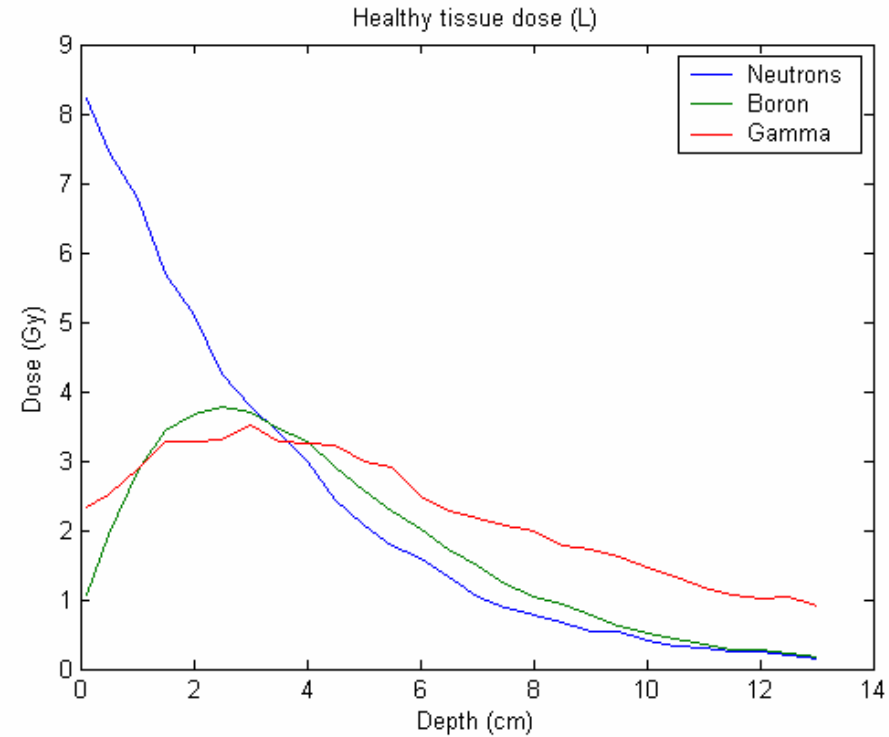

Figure 54: Components of the healthy tissue dose (L); Case 7

It is observed that the substitution of ${ }^{7} \mathrm{LiF}$ for $\mathrm{Pb}$ did not noticeably increase the Gamma-toBoron dose components but did reduce somewhat the fast-to-boron dose components ratio as compared to the SWAN reference case (Figure 32). It is concluded that $\mathrm{Pb}$ is not needed in the central and end parts of the BSA. 


\subsection{Case Eight: Fluental added to the last part of the BSA}

The purpose of Case eight is to investigate whether a reduction in the fast neutron contribution to $\mathrm{L}$ can be achieved by substituting Fluental for ${ }^{7} \mathrm{LiF}$ in the last part of the BSA. Everything else is left as in Case 2. The resulting geometry is shown in Figure 554.

The results of this system simulation are presented in Figure 55 to Figure 58. The maximum dose of $40 \mathrm{~Gy}$, at about $3 \mathrm{~cm}$ depth, is reached in 4.5 hours. This is a substantially shorter treatment time than of any previous case so far due to a high dose rate of $8.89 \mathrm{~Gy} / \mathrm{hour}$. The tumor control dose of $20 \mathrm{~Gy}$ is reached in the first $6 \mathrm{~cm}$ depth. Figure 58 shows that the fast neutron dose is still the dominant contributor to the healthy tissue dose.

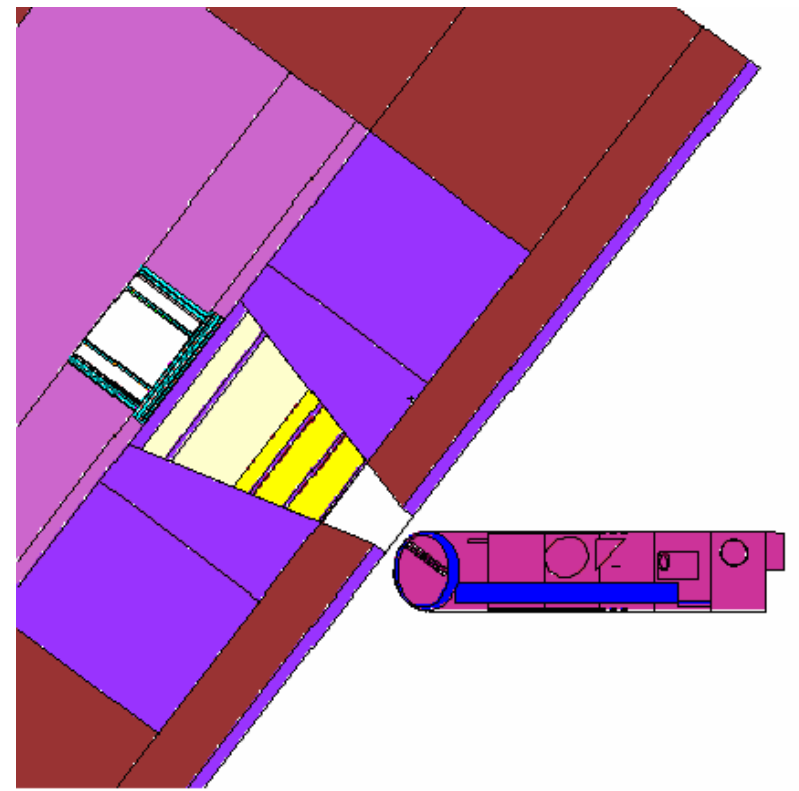

Figure 55: Cross sectional view of the system in Case 8 


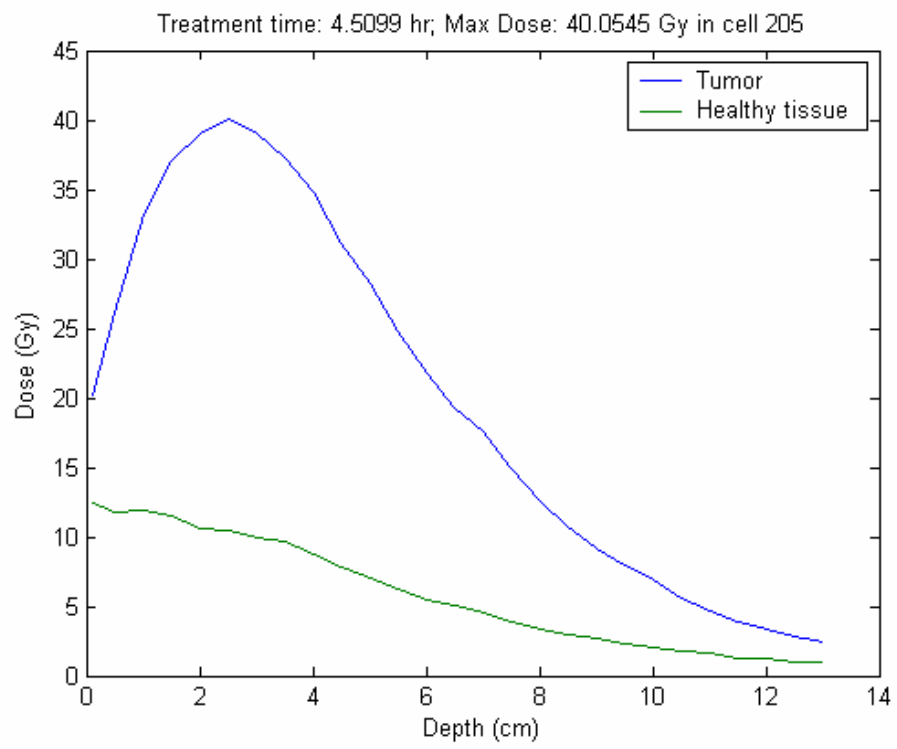

Figure 56: Total dose to the healthy tissue and to the tumor as a function of depth; Case 8

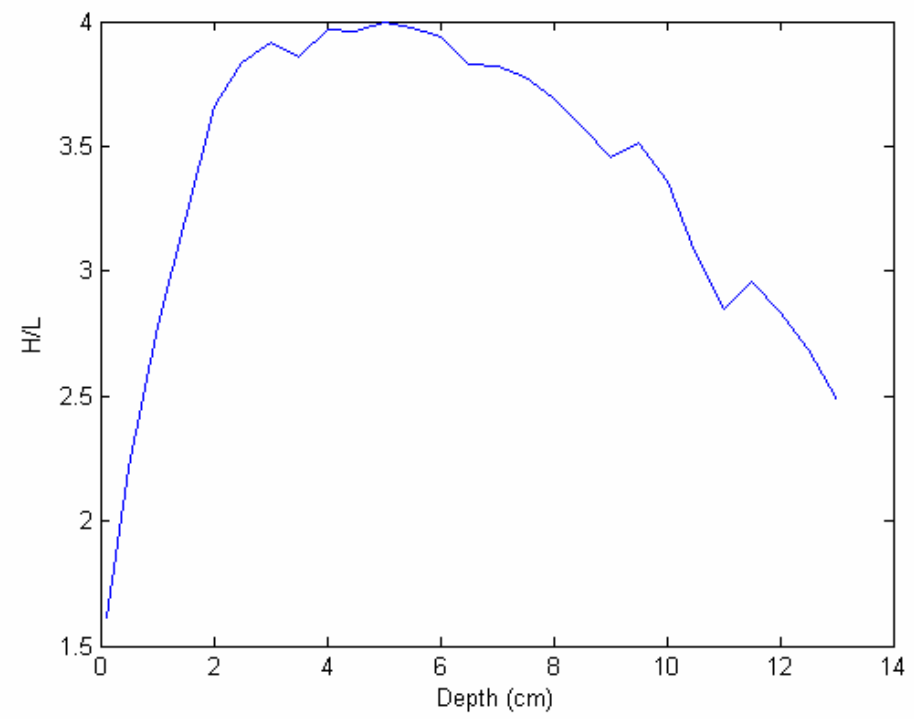

Figure 57: Tumor-to-healthy tissue dose ratio (H/L); Case 8 


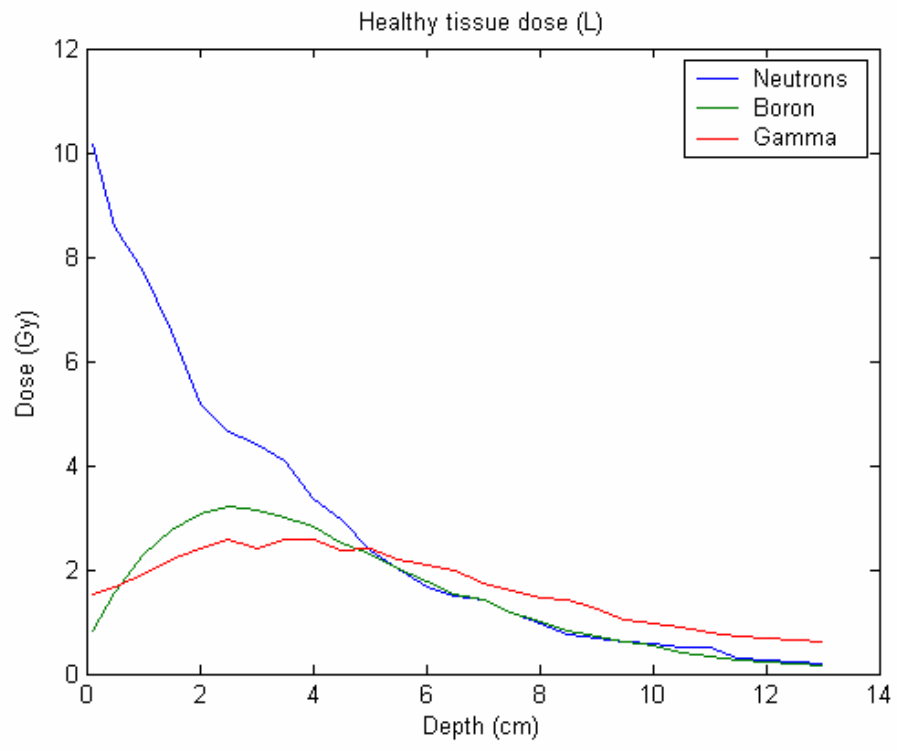

Figure 58: Components of the healthy tissue dose (L); Case 8

\subsection{Case Nine: Like Case One with Pb layer added at the exit of the BSA}

The purpose of this case is to find out whether a thicker Pb filter at the end of the BSA can substantially reduce the contribution of the gamma rays to the healthy tissue dose (L). A cross sectional view of this system is shown in Figure 59 while Figure 60 shows an enlargement of the end part of the BSA. 


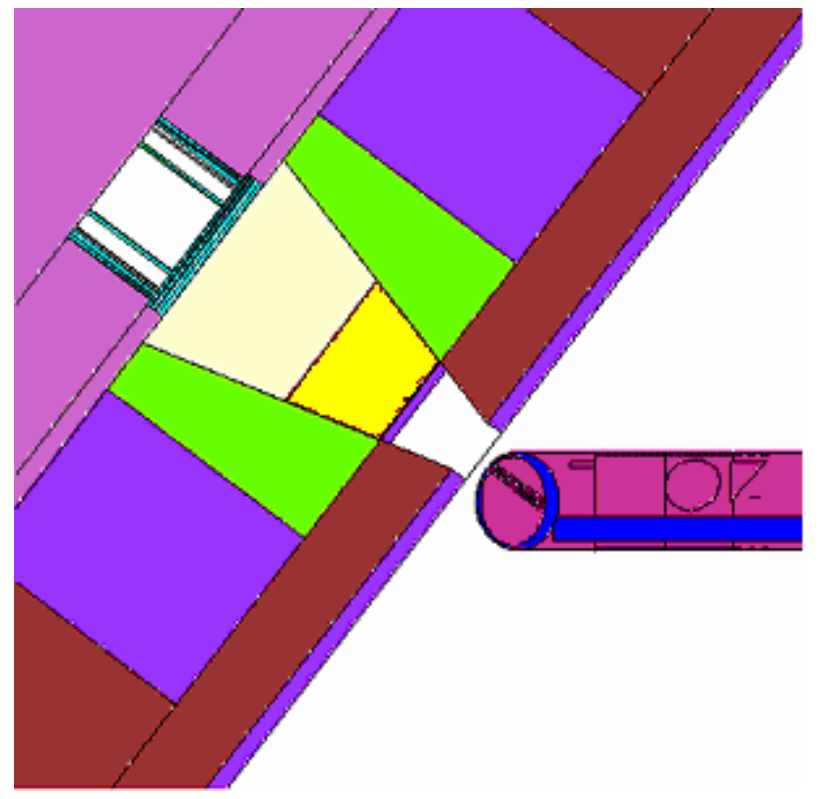

Figure 59: Cross sectional view of the system in Case 9

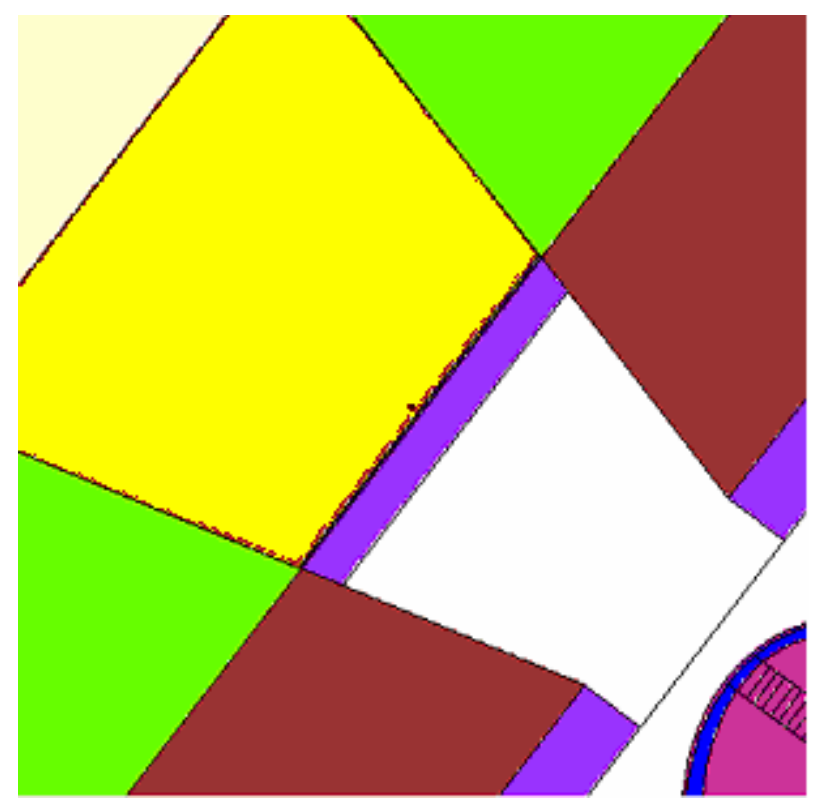

Figure 60: Enlarged end part of Case 9 BSA showing the thick $\mathrm{Pb}$ layer added at the exit BSA

The lead thickness is determined so as to cut down the gamma component to about half the value of the reference case. Figure 60 shows the absorption (in dark blue) and attenuation (in pink) coefficients of lead as a function of the gamma ray energy. The light blue curve gives the thickness necessary to cut the gamma current by a factor of 2.5 , neglecting the buildup factor. A lead thickness of $2 \mathrm{~cm}$ was chosen for analysis. 
The results obtained are plotted in Figure 62 to Figure 64. The maximum dose of 36.4 Gy is reached with an exposure time of little less than 9 hours at a depth of about $3 \mathrm{~cm}$. The dose rate is $4 \mathrm{~Gy} /$ hour. Figure 643 shows that the lead end layer is not very effective in cutting the gamma dose substantially, indicating that a substantial contribution to the gamma dose comes from the sided of the collimator.

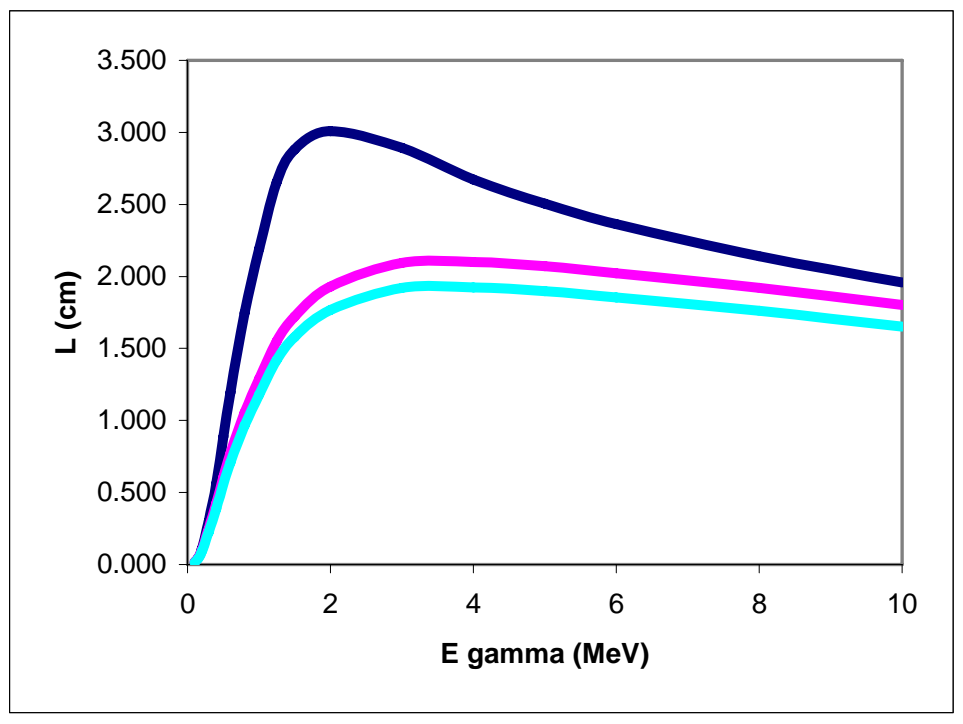

Figure 61: Absorption (dark blue) and attenuation coefficient (pink) of lead for gamma rays: in light blue the thickness necessary to cut the gamma component by a factor of 2.5 , neglecting the buildup factor. 


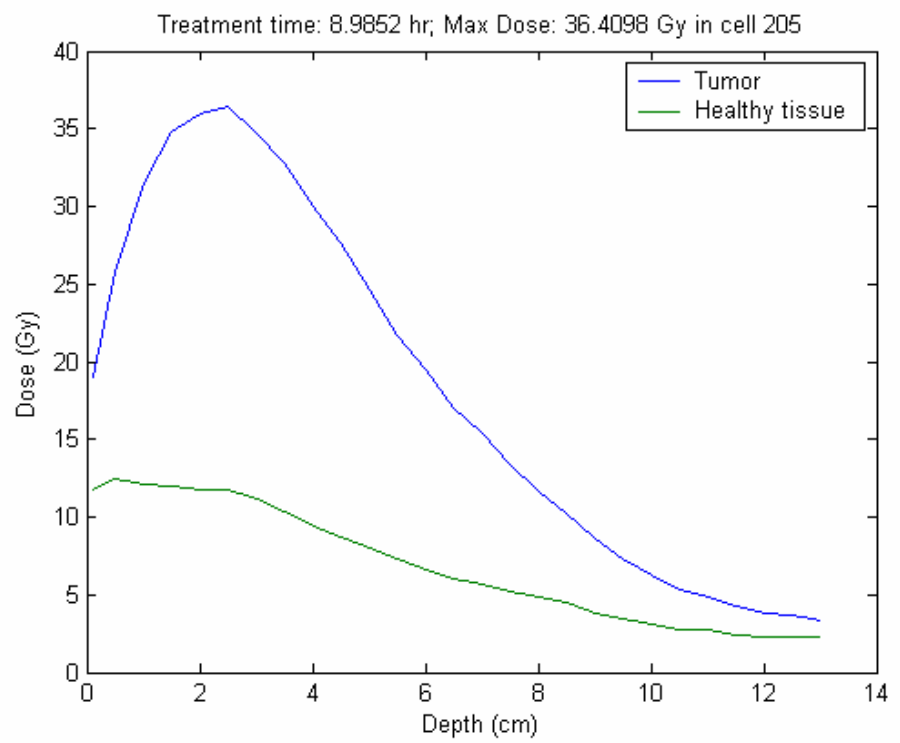

Figure 62: Total dose to the healthy tissue and to the tumor as a function of depth; Case 9

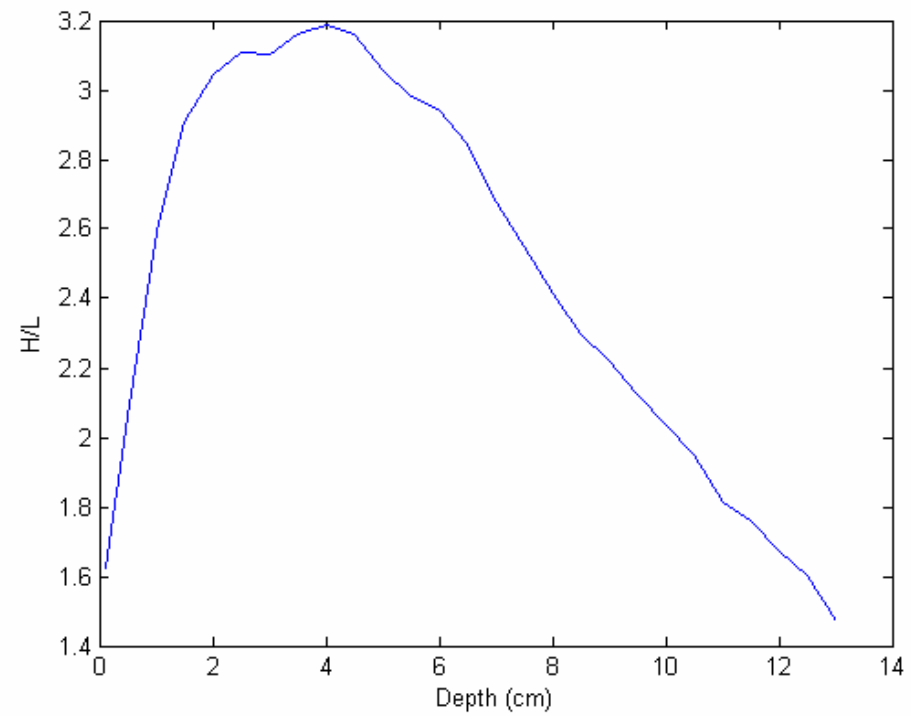

Figure 63 Tumor-to-healthy tissue dose ratio (H/L); Case 9 


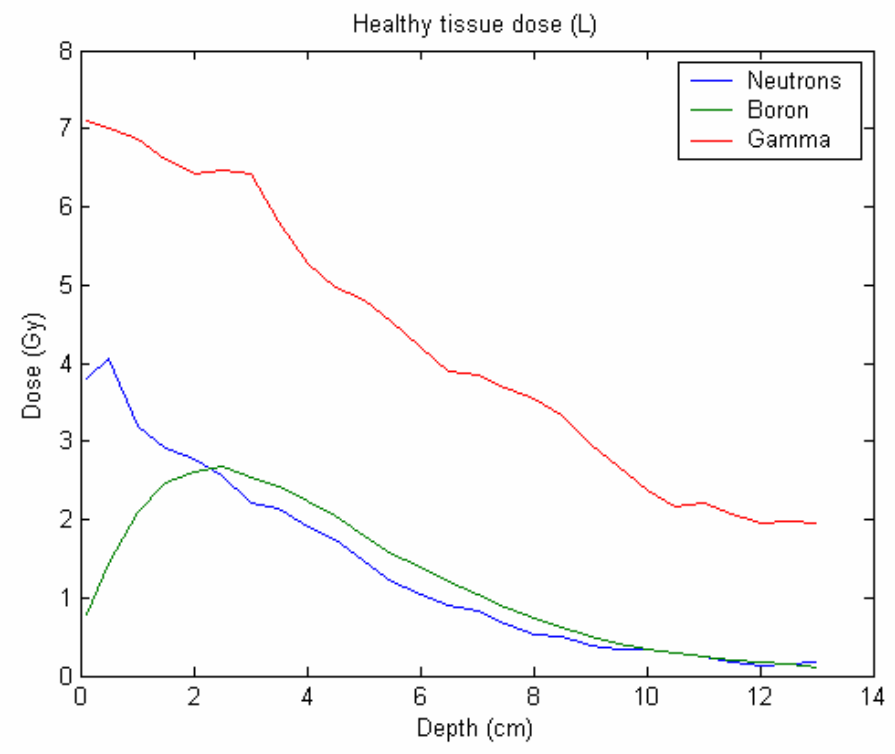

Figure 64: Components of the healthy tissue dose (L); Case 9

\subsection{Case Ten: Like Case 9 but with a Pb collar added}

This case is designed to check the above hypothesis by adding a "collar" of lead around the collimator walls as shown in Figure 65. The collar thickness is $2.1 \mathrm{~cm}$ the same as the Pb layer thickness at the exit of the BSA.

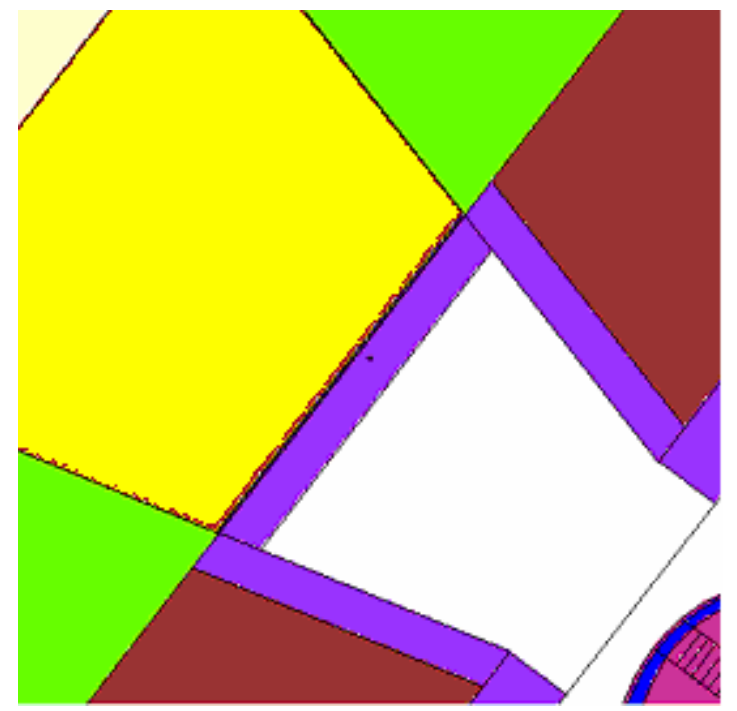

Figure 65: Cross sectional view of the end part of the system in Case 10 
Figure 66 to Figure 68give the results for this case: the maximum dose of 47.5 Gy at about 2$2.5 \mathrm{~cm}$ depth is reached with an exposure time of 10.3 hours. The dose rate is $4.6 \mathrm{~Gy} / \mathrm{hour}$, which is a substantial improvement compared to Case nine without the collar. Comparing Figure 68 and Figure 64 it is found that, as expected, the addition of lead collar substantially reduces the gamma dose; it contribution to the skin dose is now comparable to that of the fast neutrons.

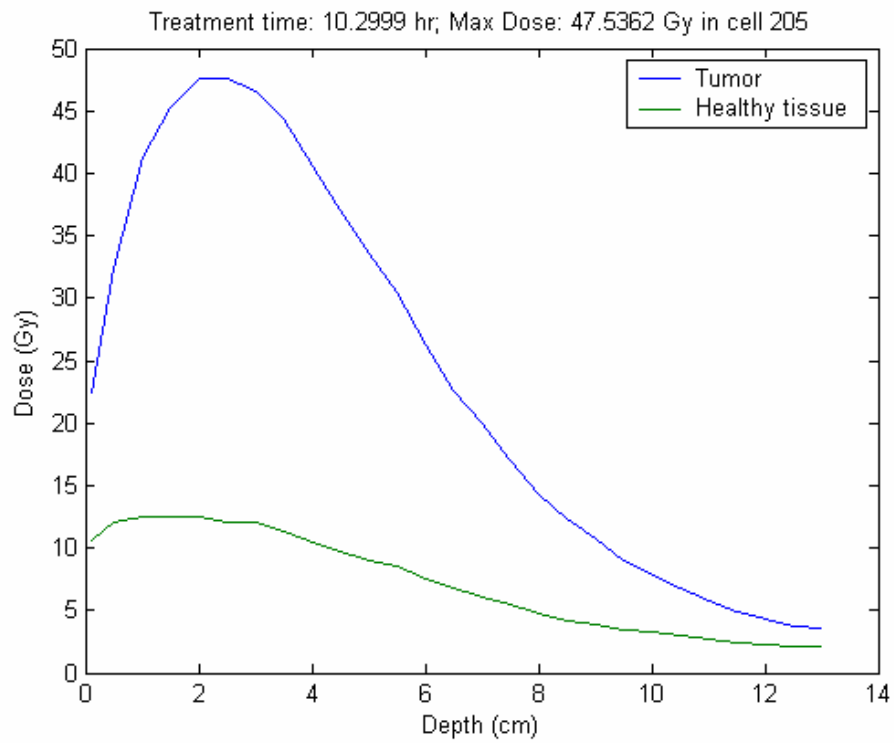

Figure 66: Total dose to the healthy tissue and to the tumor as a function of depth; Case 10

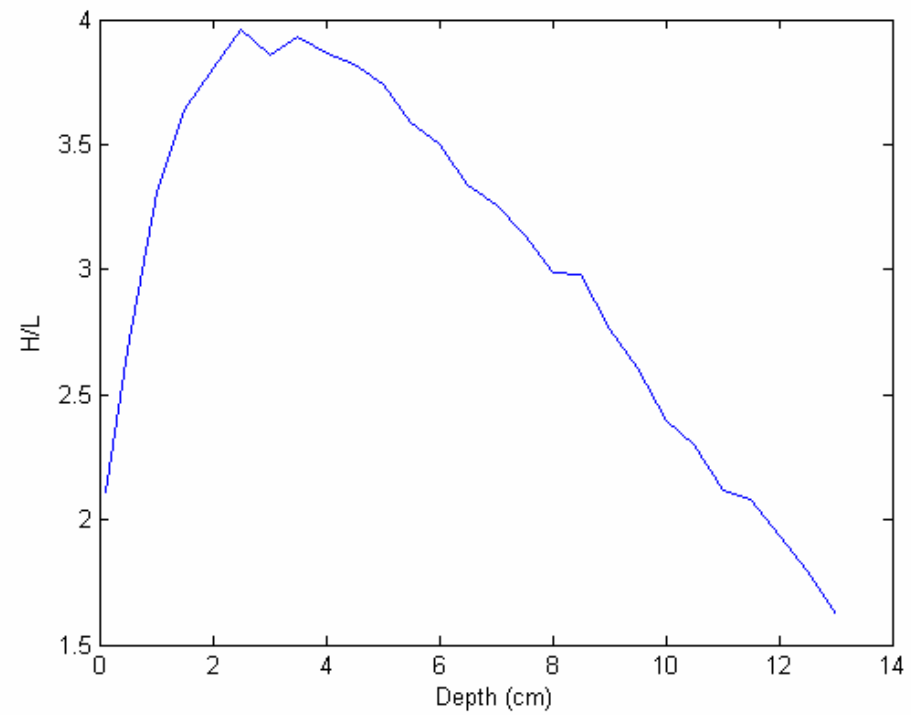

Figure 67: Tumor-to-healthy tissue dose ratio (H/L); Case 10 


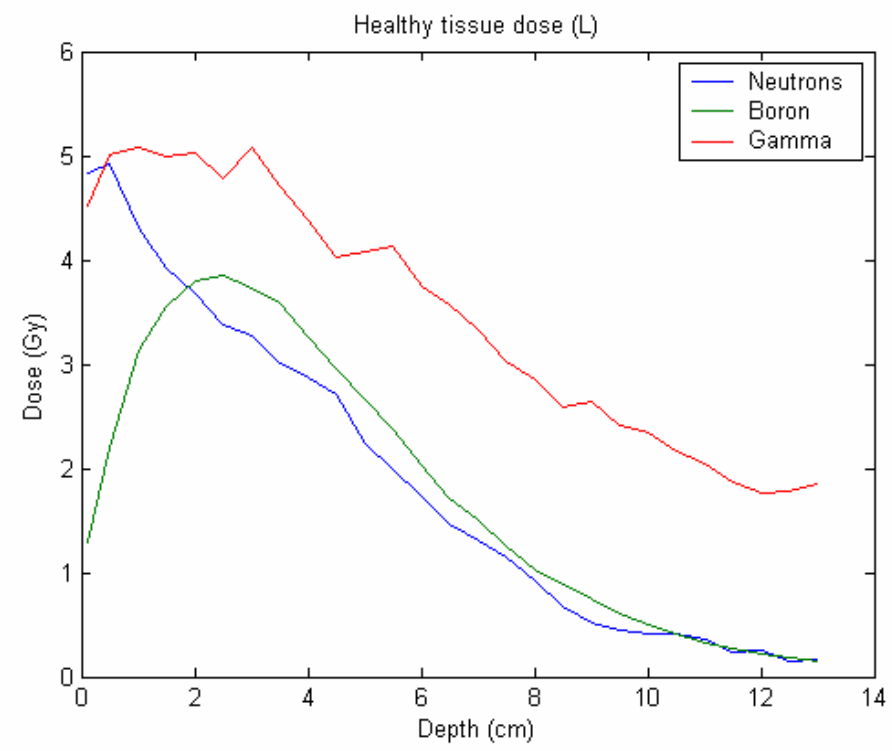

Figure 68: Components of the healthy tissue dose (L); Case 10

\subsection{Case Eleven: Like Case Eight with a lead "collar"}

Having found (Case Ten) that the lead collar is very effective in reducing the gamma dose, such a collar is added to Case Eight system that gave the best performance so far. A cross sectional view of the resulting system is shown in Figure 69 and Figure 69. The results are presented in Figure 71 to Figure 73 . The maximum dose of $34.7 \mathrm{~Gy}$ at about $3 \mathrm{~cm}$ depth is achieved with an exposure of about 3.43 hours. The dose rate is $10.12 \mathrm{~Gy} /$ hour - the largest obtained so far, and the tumor control dose is achieved everywhere up to $6 \mathrm{~cm}$ depth. As in Case 8 , the fast neutron component (Figure 73) is the major contributor to the skin dose. 


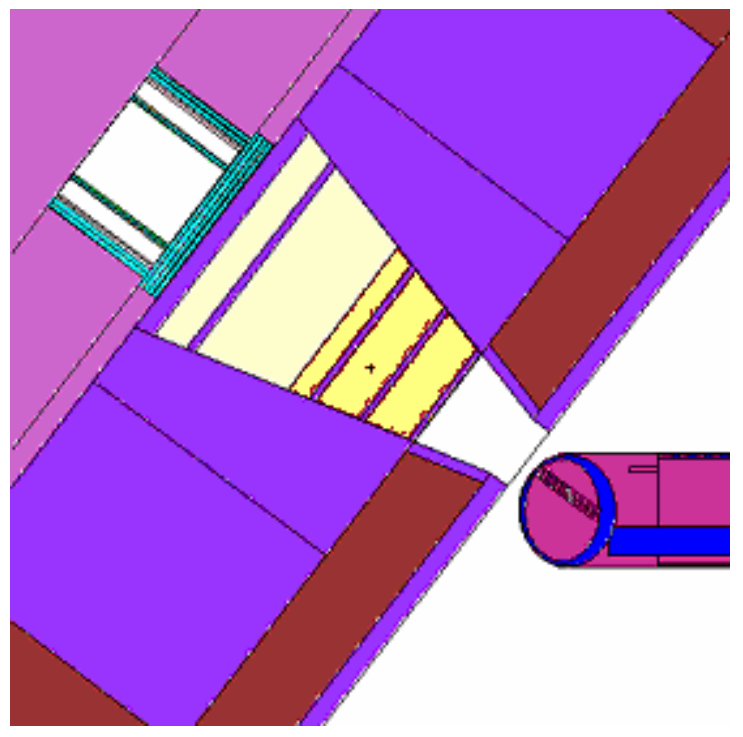

Figure 69: Cross sectional view of the system in Case 11

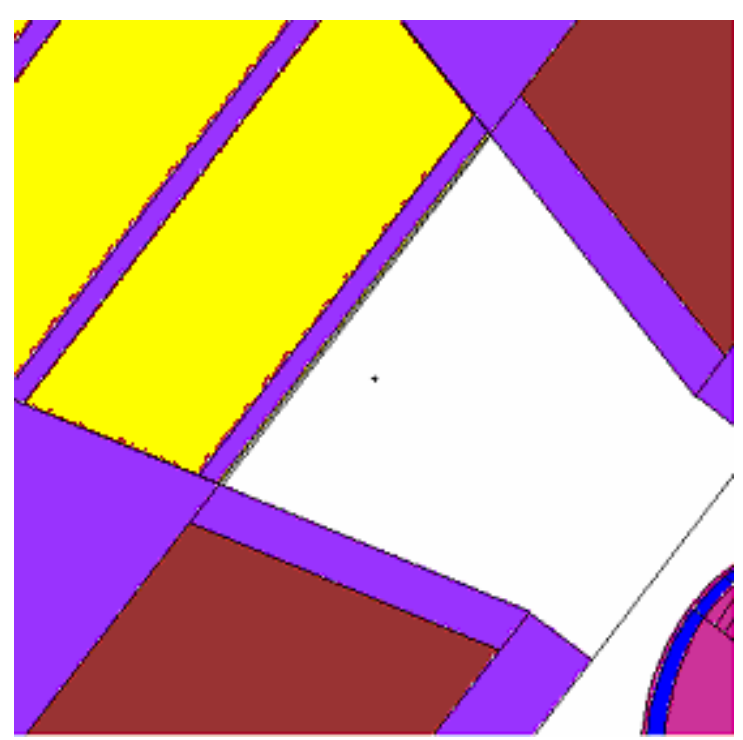

Figure 70: Cross sectional view of the collimator region of Case 11 system 


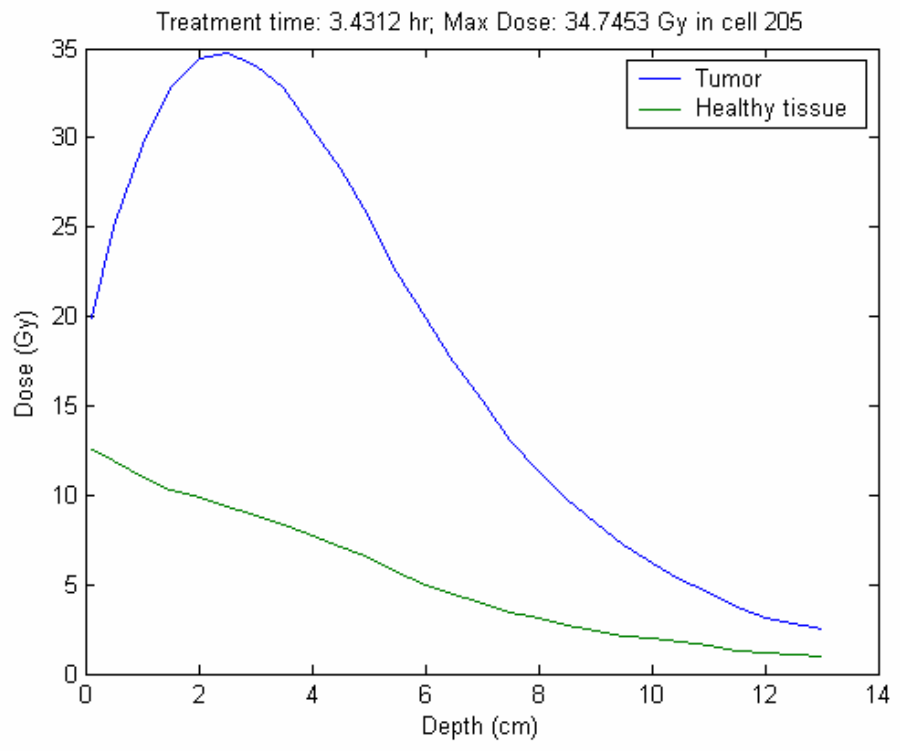

Figure 71: Total dose to the healthy tissue and to the tumor as a function of depth; Case 11

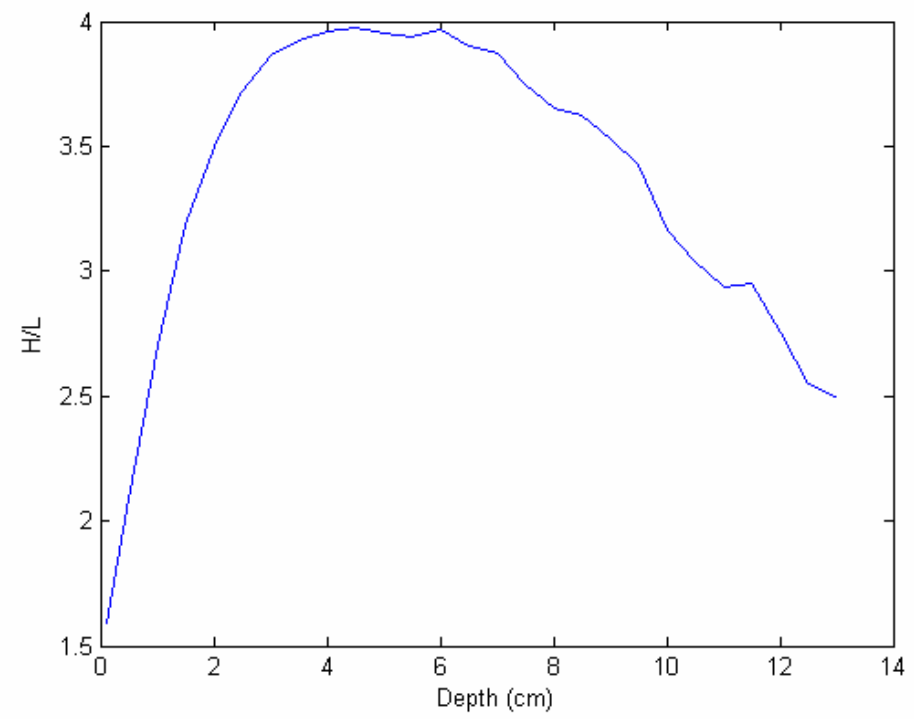

Figure 72: Tumor-to-healthy tissue dose ratio (H/L); Case 11 


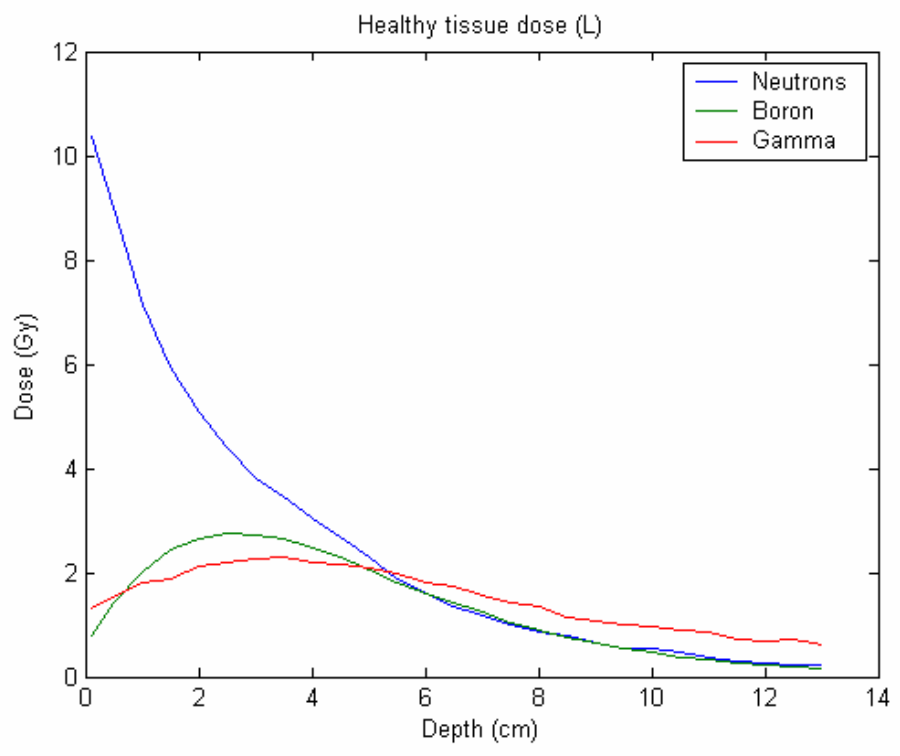

Figure 73: Components of the healthy tissue dose (L); Case 11

\subsection{Case Twelve: Like Case One but ${ }^{6} \mathrm{LiF}$ extended up to the SCM}

The purpose of Case Twelve is to find out whether an extension of the $0.25 \mathrm{~mm}$ layer of ${ }^{6} \mathrm{LiF}$ between the BSA and the reflector is responsible for the large difference in the gamma and neutron dose components between Cases One and Case Two. This layer was originally aimed at reducing the flux of thermal neutrons that reach the reflector and generate capture gamma rays. In the original Goff's design this layer extends on the entire side of the BSA up to the SCM. In Case Twelve, as in Case One, the ${ }^{6} \mathrm{LiF}$ is eliminated from the $30 \mathrm{~cm}$ closer to the $\mathrm{SCM}$ so as to minimize the ${ }^{6} \mathrm{Li}$ effect on the SCM $\mathrm{k}_{\text {eff. }}$ The resulting design considered is shown in Figure 743 and in Figure 754 in which the thin ${ }^{6} \mathrm{LiF}$ layer in shown in yellow.

The simulation results of this system are summarized in Figure 765 and Figure 7776. The exposure time was not properly evaluated because the size of the SCM was not adjusted to compensate for the poisoning effect of the added ${ }^{6} \mathrm{LiF}$. As a result $\mathrm{k}_{\mathrm{eff}}$ is lower than 0.98 and the exposure time is increased. Nevertheless, Figure 77 shows that, although the gamma component is somewhat smaller than in the reference system (Figure 25), it is still the dominant, indicating that the extra ${ }^{6} \mathrm{LiF}$ is not responsible for the substantial cut in the gamma dose component experienced in Case 2. 


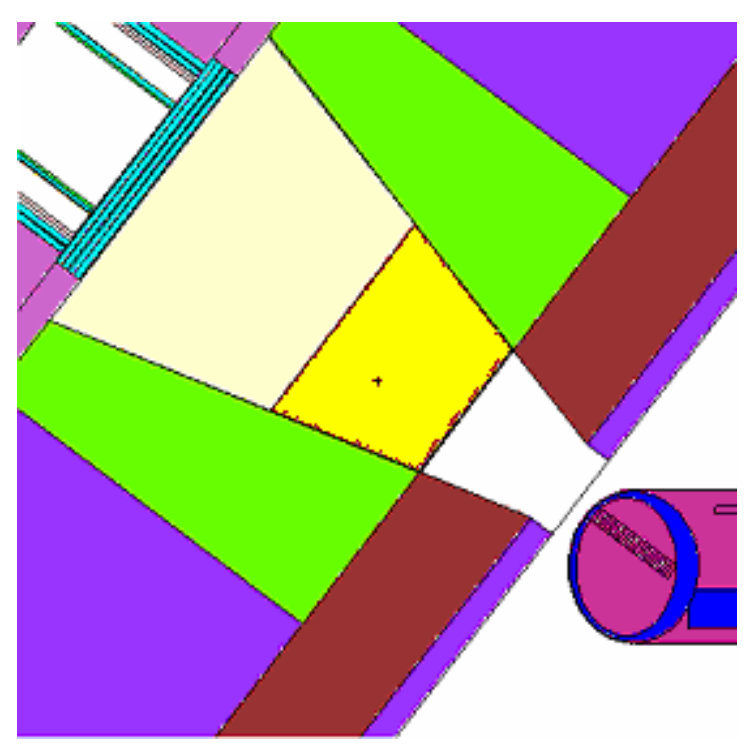

Figure 74: Cross sectional view of the system of Case 12

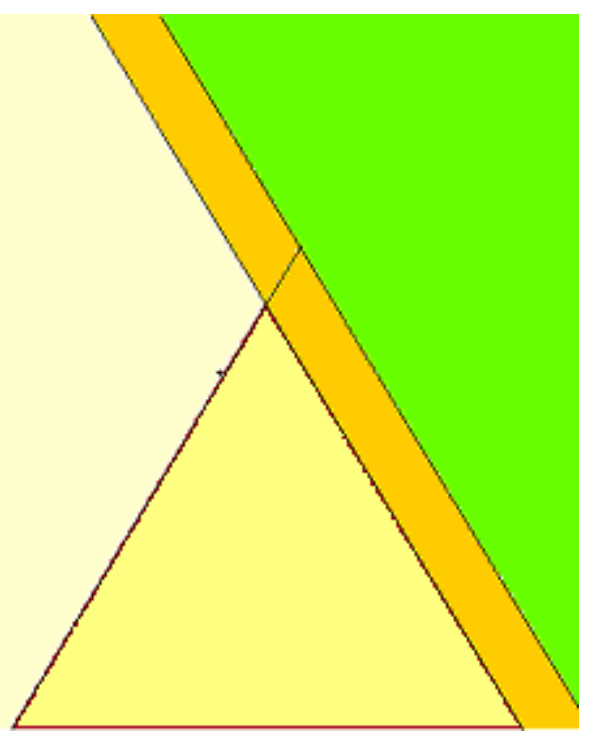

Figure 75: Section of Case $12 \mathrm{BSA}$ showing the $0.25 \mathrm{~mm}{ }^{6} \mathrm{LiF}$ layer (in yellow) between the BSA (in white and red) and the alumina reflector (in green). 


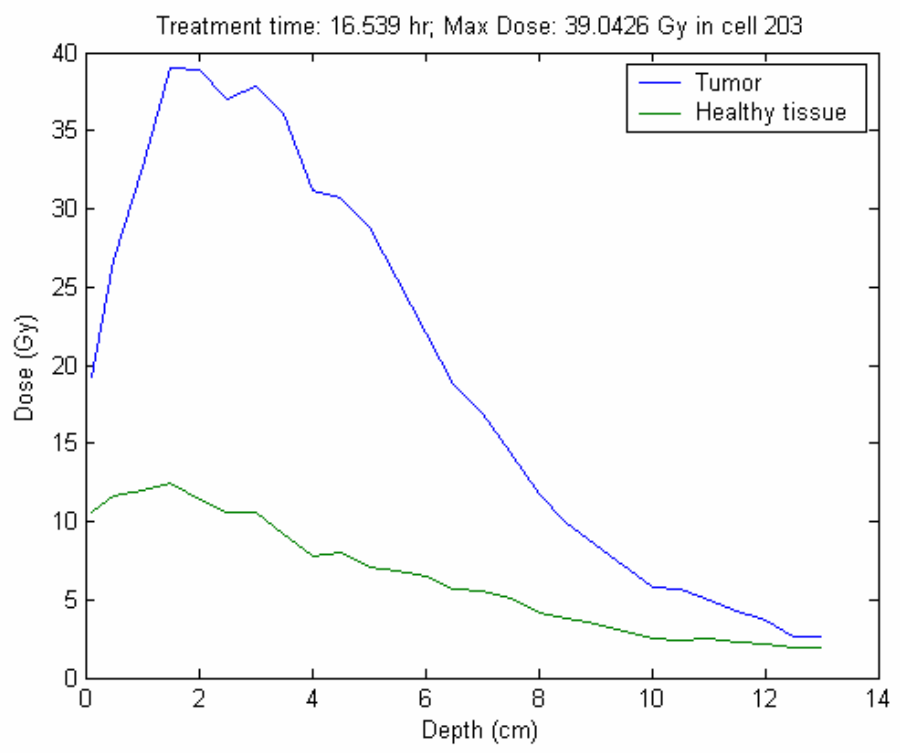

Figure 76: Total dose to the healthy tissue and to the tumor as a function of depth; Case 12.

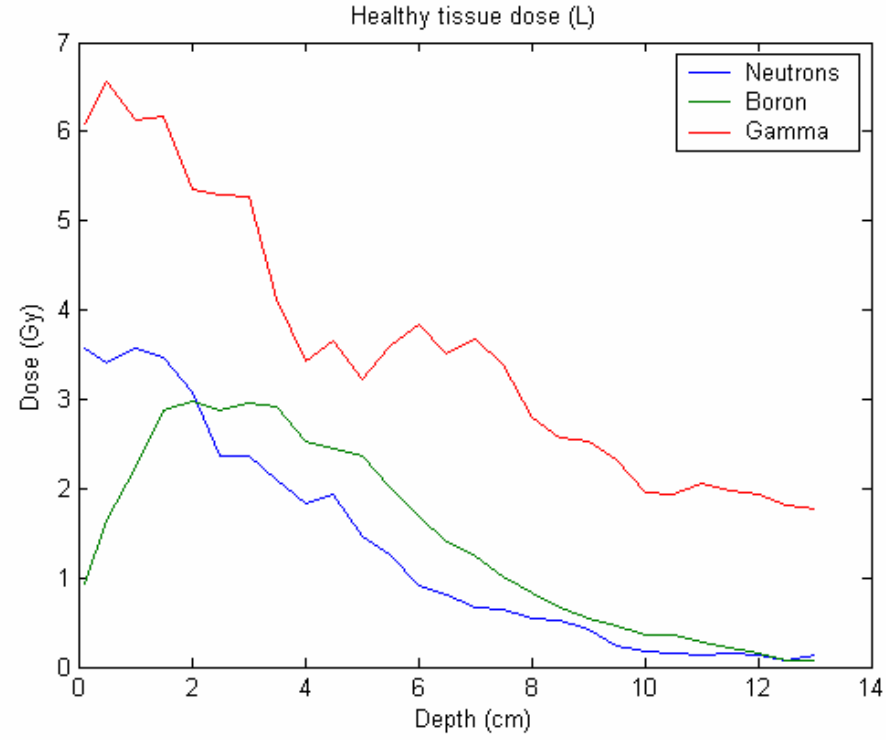

Figure 77: Components of the healthy tissue dose (L); Case 12

\subsection{Case Thirteen: BeO instead of lead reflector for the BSA}

The purpose of this case is to evaluate the extent to which the fast neutron component contribution can be reduced by replacing the lead reflector of the BSA with $\mathrm{BeO}$. The design is the same as of Case 11. It was found that, due to the better neutron reflection properties of $\mathrm{BeO}$, the size of the SCM could be substantially reduced. Two different approaches were examined for 
maintaining $\mathrm{k}_{\text {eff }}=0.98$ : in Case Thirteen one vertical fuel plate was removed and the radius of the remaining fuel plates was reduced from $17.0 \mathrm{~cm}$ to $16.5 \mathrm{~cm}$. The resulting system is shown in Figure 78. In Case Fourteen the four vertical fuel plates were maintained and their radius was reduced. The simulation results are plotted in Figure 79 to Figure 81.

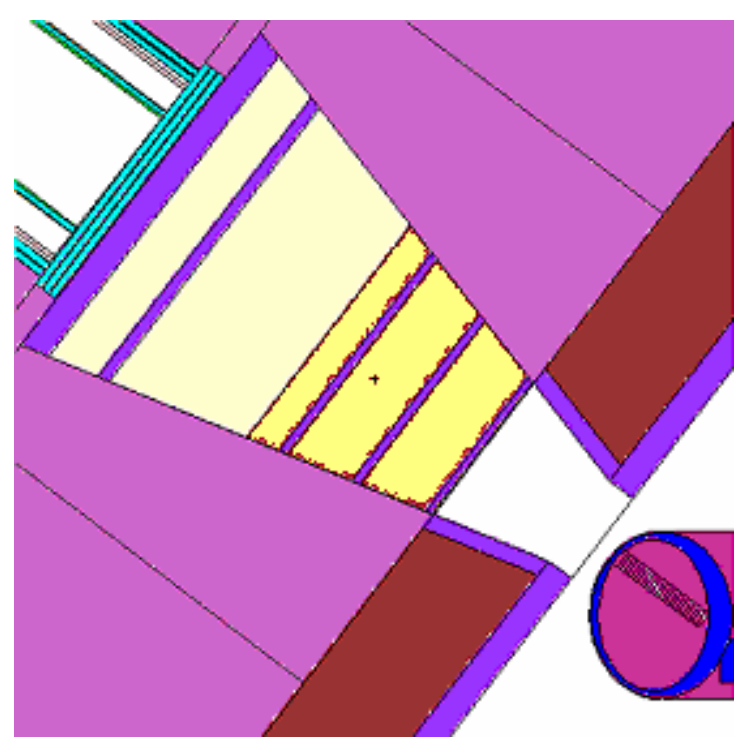

Figure 78: Cross sectional view of Case Thirteen system; the $\mathrm{BeO}$ reflector is in violet

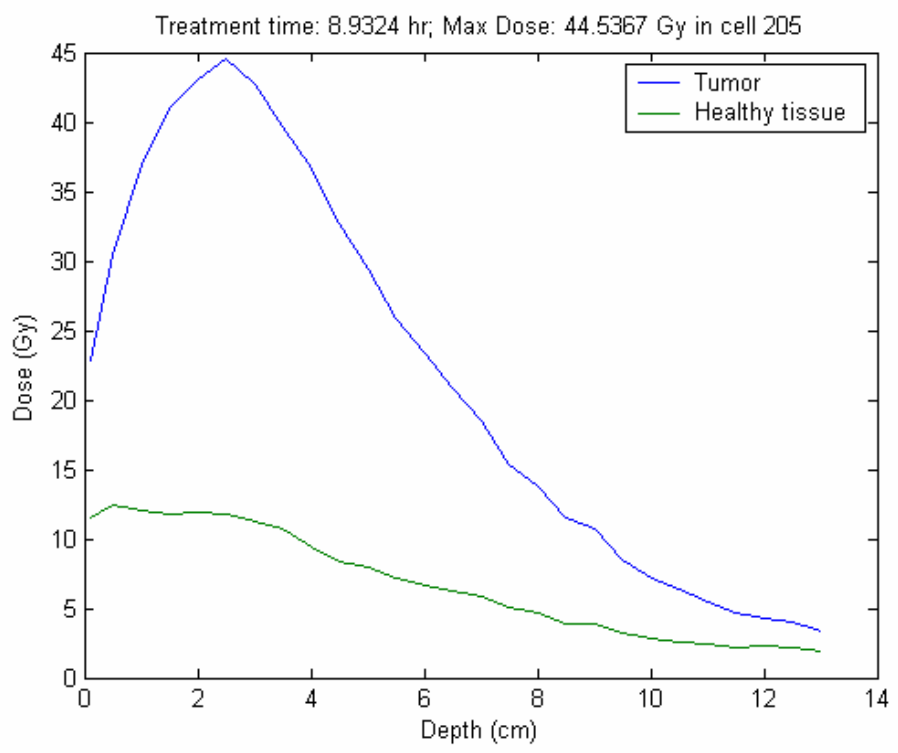

Figure 79: Total dose to the healthy tissue and to the tumor as a function of depth; Case 13 


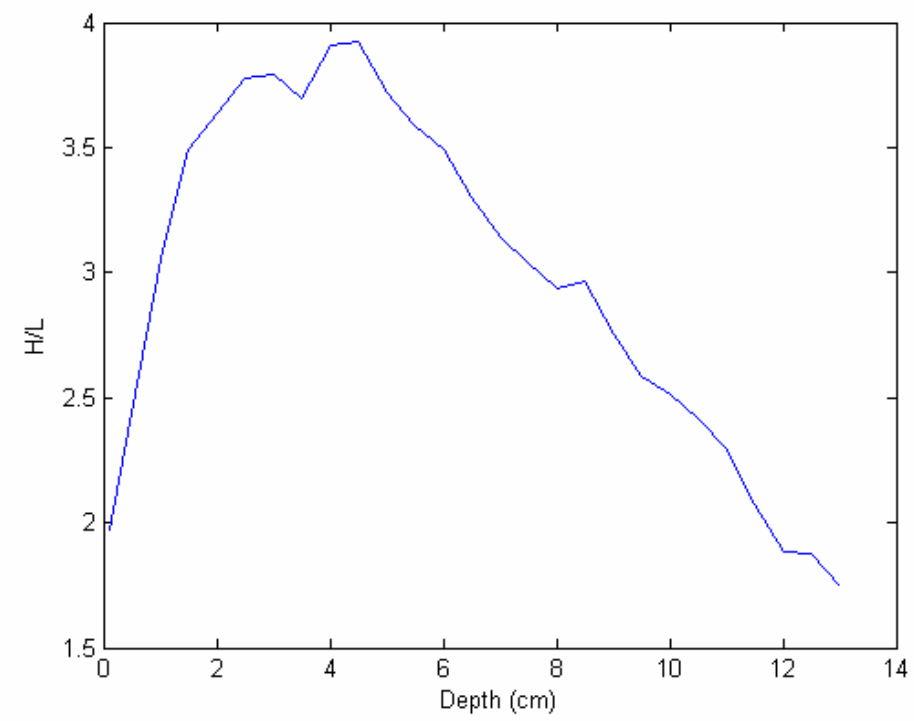

Figure 80: Tumor-to-healthy tissue dose ratio (H/L); Case 13

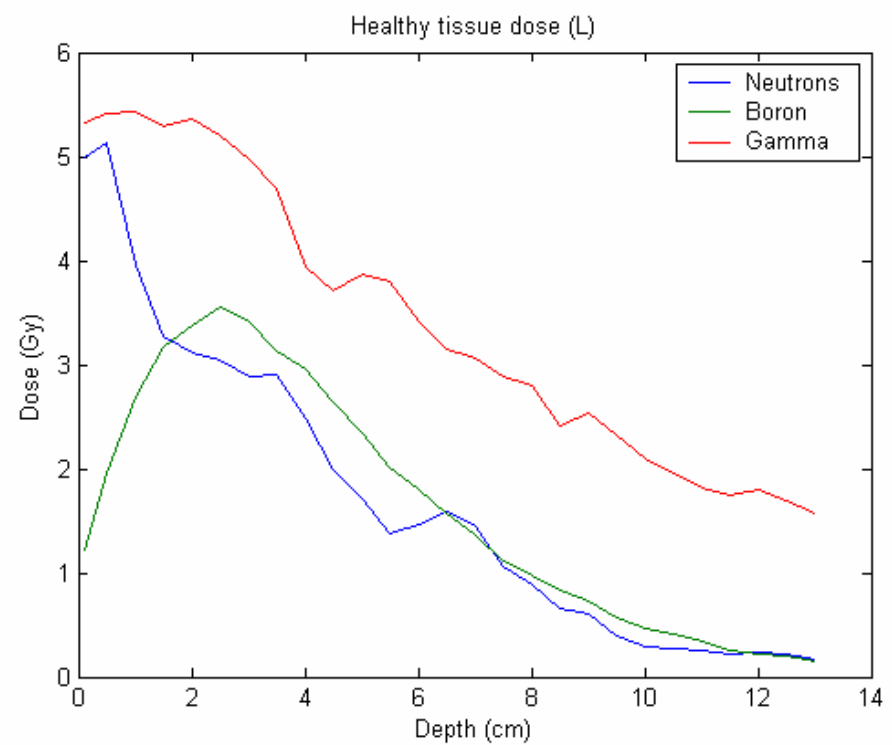

Figure 81: Components of the healthy tissue dose (L); Case 13

The maximum tumor dose of 44.5 Gy is reached at about $2.5 \mathrm{~cm}$ depth with an irradiation of 8.9 hours. The dose rate is $4.5 \mathrm{~Gy} /$ hour. The fast neutron component is substantially reduced but the performance measured by the dose rate is drastically worse than in Case 11 . 


\subsection{Case Fourteen: BeO instead of Alumina reflector for the BSA; Part II}

This case is similar to Case Thirteen with the exception that the four vertical fuel plates of the $\mathrm{SMC}$ are maintained but their radius is reduced to $13.91 \mathrm{~cm}$ to meet the $\mathrm{k}_{\mathrm{eff}}=0.98$ constraint. The resulting system layout is shown in Figure 82. The results obtained for this system are shown in Figure 83 to Figure 85.

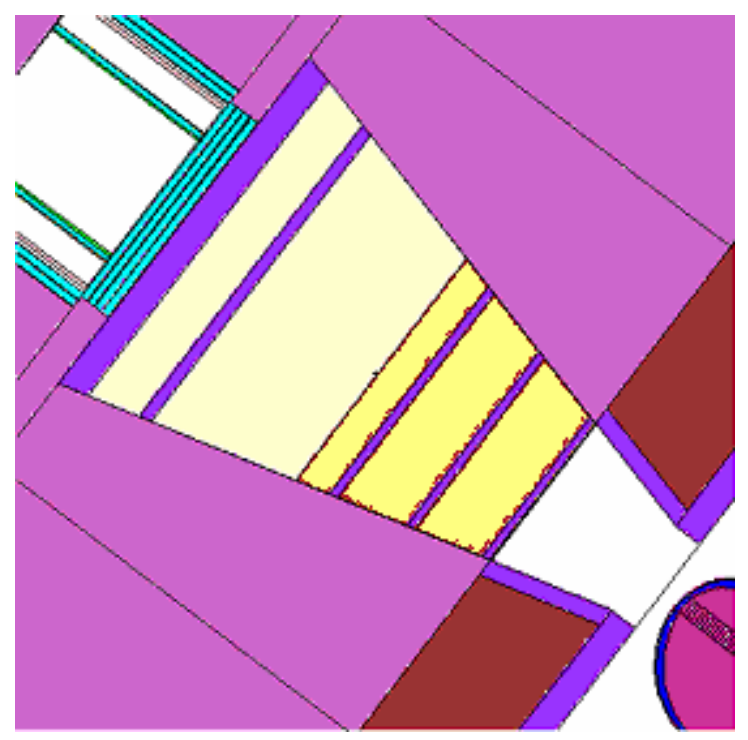

Figure 82: Cross sectional view of the system in Case 14

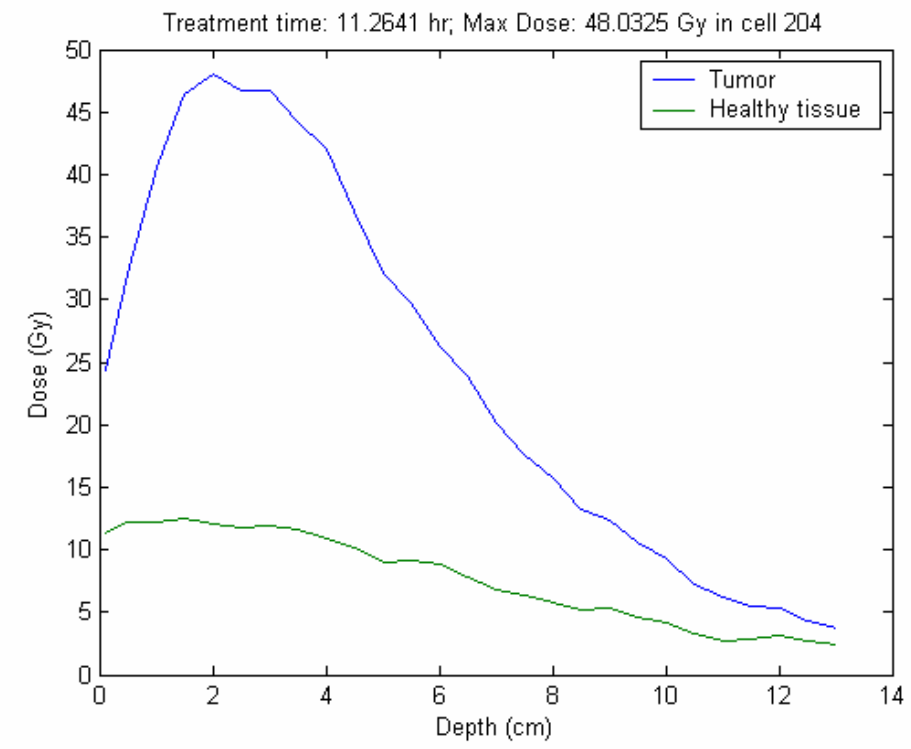

Figure 83: Total dose to the healthy tissue and to the tumor as a function of depth; Case 14 


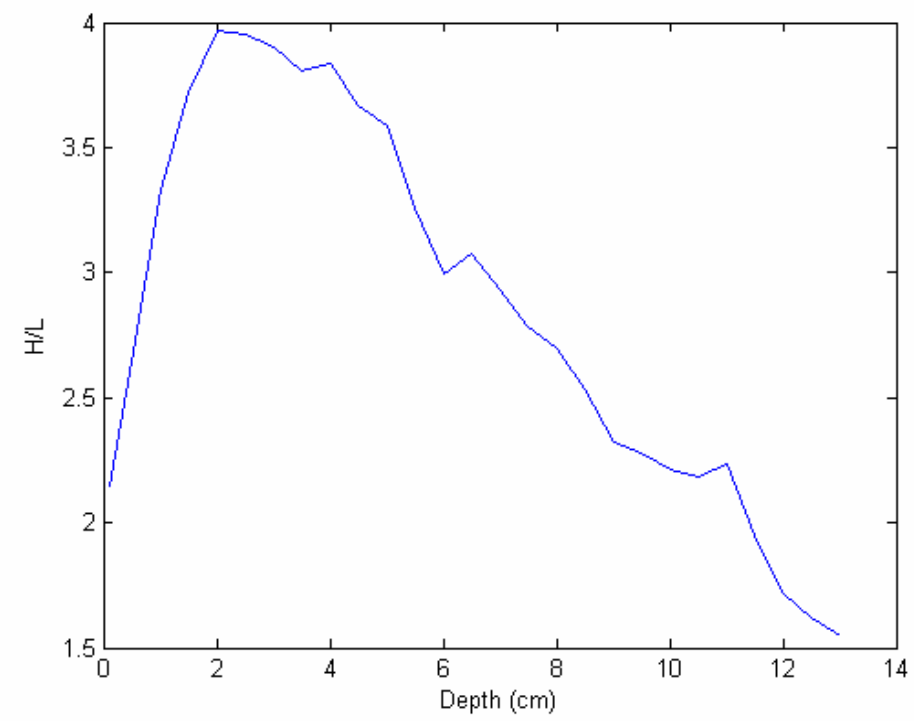

Figure 84: Tumor-to-healthy tissue dose ratio (H/L); Case 14

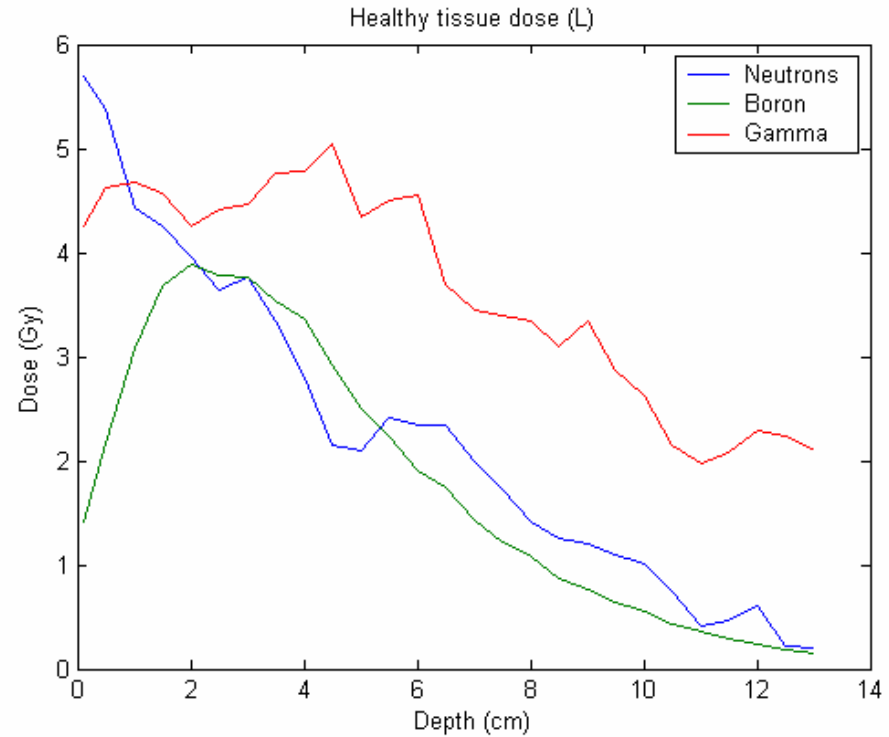

Figure 85: Components of the healthy tissue dose (L); Case 14

The maximum dose of 48 Gy is reached at about $2 \mathrm{~cm}$ depth with an irradiation time of about 11.26 hours. The dose rate is $4.3 \mathrm{~Gy} /$ hour. The performance of this system is even worse than that of Case Thirteen. However, the gammas and neutrons contribution to the skin dose is well balanced. 


\subsection{Case Fifteen: No lead in the BSA}

The purpose of this case is to investigate the role of the second lead slab on the relative contribution of the gamma and neutron components to the dose. For this purpose only the lead slab closest to the SCM is kept. The thickness of the fluental layer is the same as in the reference BSA (Case One). The BSA reflector is made of lead. The collimator configuration is the same as in Case Ten. Figure 86 shows the resulting system. The results are shown in Figure 87 to Figure 89.

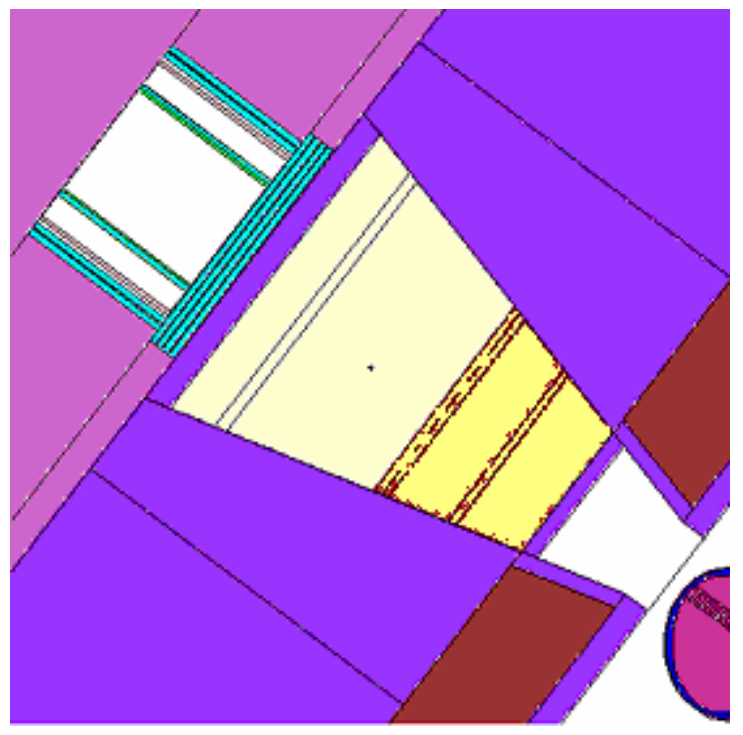

Figure 86: Cross sectional view of Case 15 system

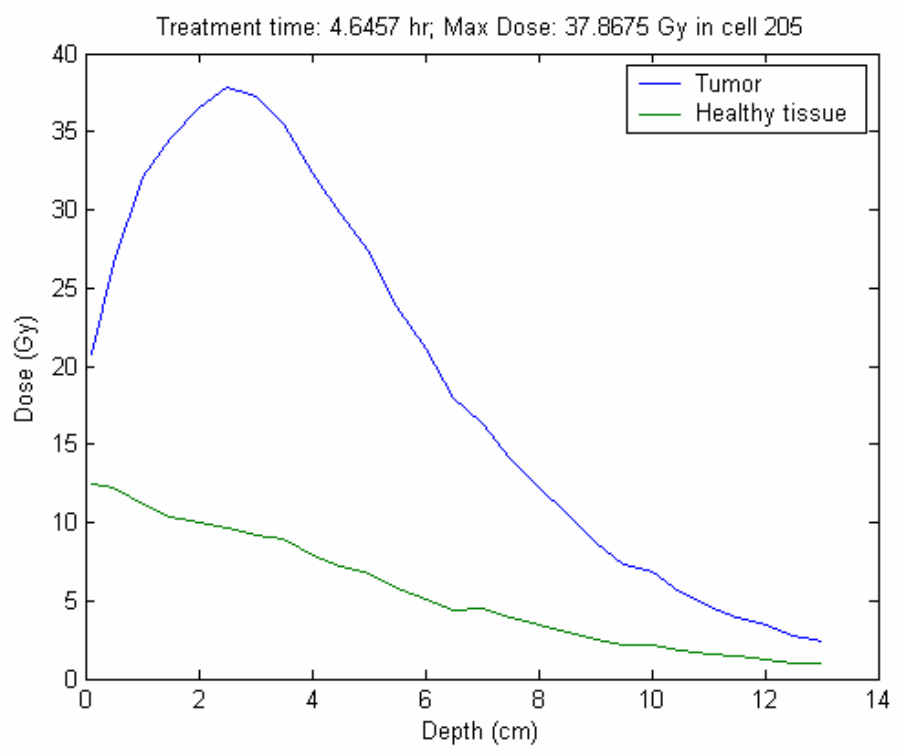

Figure 87: Total dose to the healthy tissue and to the tumor as a function of depth; Case 15 


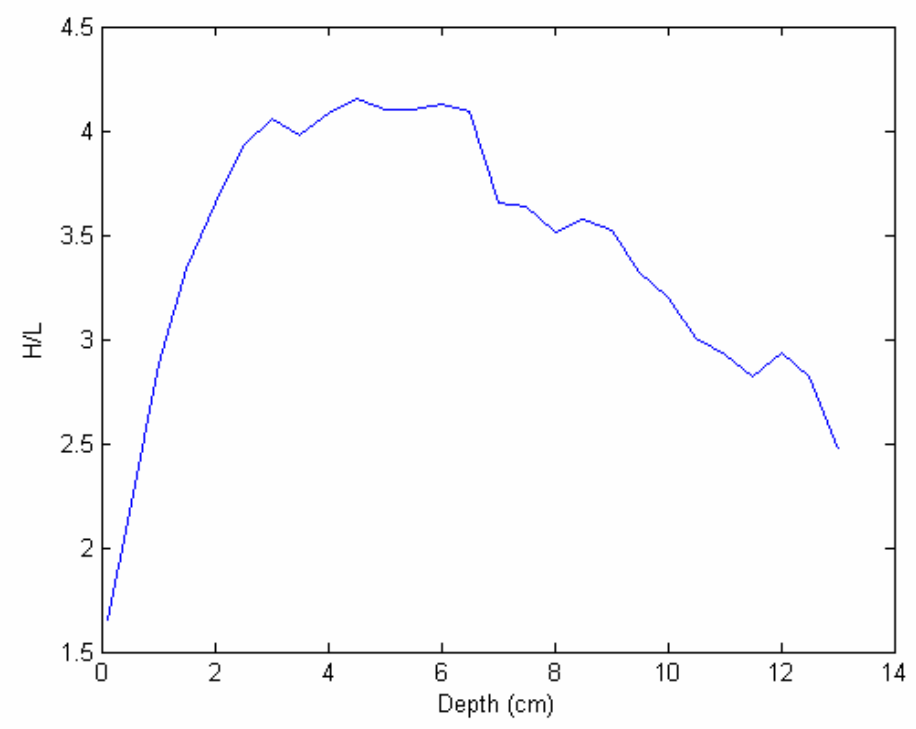

Figure 88: H/L (Tumor/healthy dose); Case 15

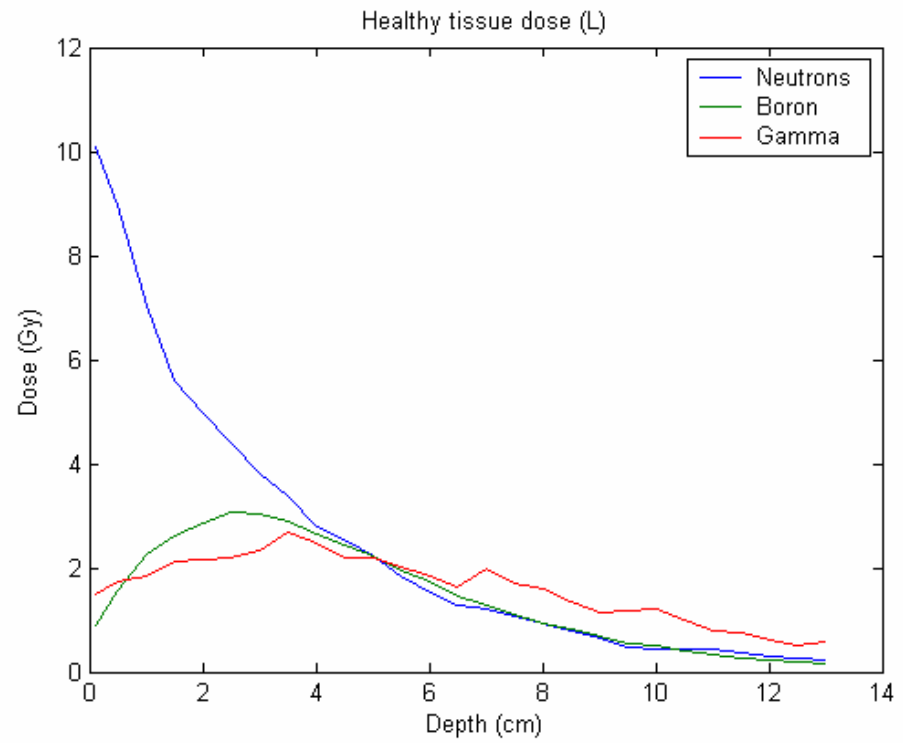

Figure 89: Components of the healthy tissue dose (L); Case 15

The maximum dose of about $37.8 \mathrm{~Gy}$ is reached at $2.5 \mathrm{~cm}$ depth in about 4.65 hours. The dose rate is $8.1 \mathrm{~Gy} /$ hour. However, the fast neutron contribution is still the main component to the skin and skull dose. 


\subsection{Case Sixteen: As Case Fifteen but using alumina reflector}

The purpose of this case is to confirm what appears to be the conclusion from the previous cases that the alumina BSA reflector is primarily responsible for the balanced contribution of the neutrons and the gammas to the healthy tissue dose in the reference design ${ }^{6}$ (see Figure 68). For doing so the lead reflector of Case Fifteen is replaced with the alumina reflector used in Goff's design. Figure 90 shows the layout of the resulting system and Figure 91 to Figure 93 give the results.

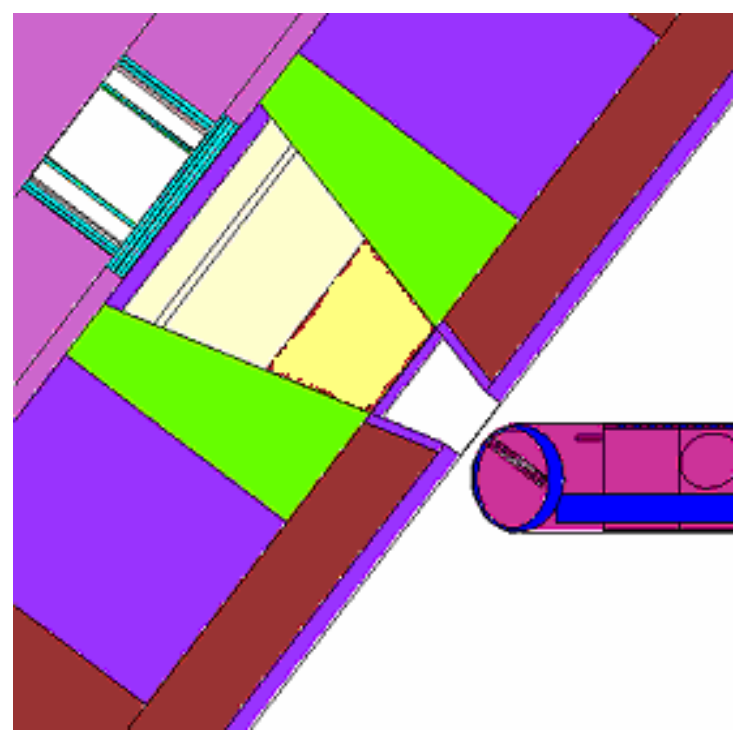

Figure 90: Cross sectional view of Case 16 system

${ }^{6}$ Case 1 and its improvements through the use of the lead collar, Cases Nine and Ten 


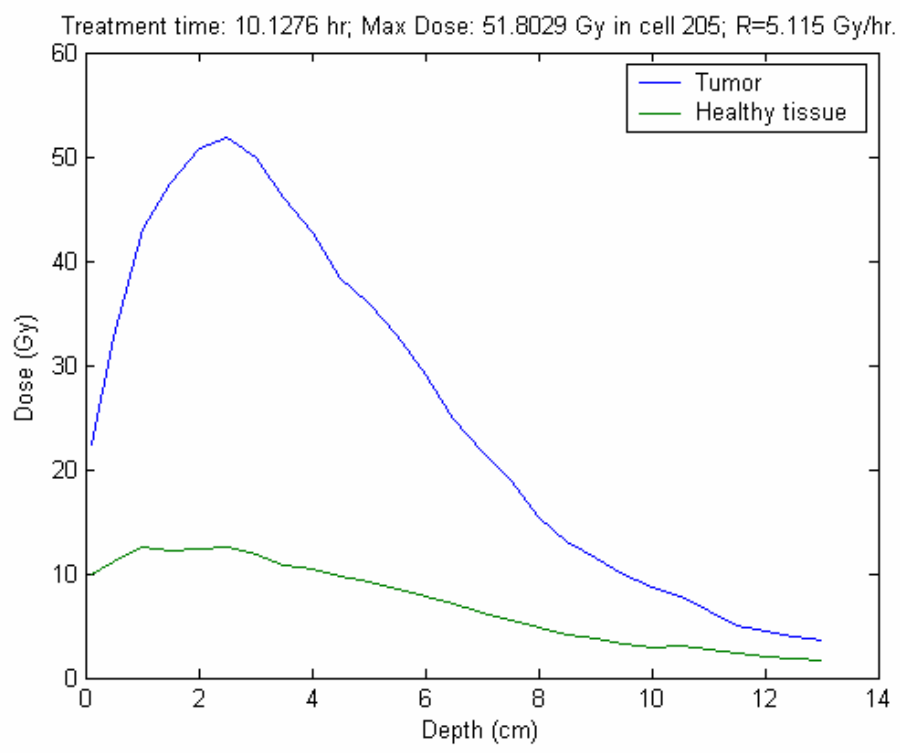

Figure 91: Total dose to the healthy tissue and to the tumor as a function of depth; Case 16

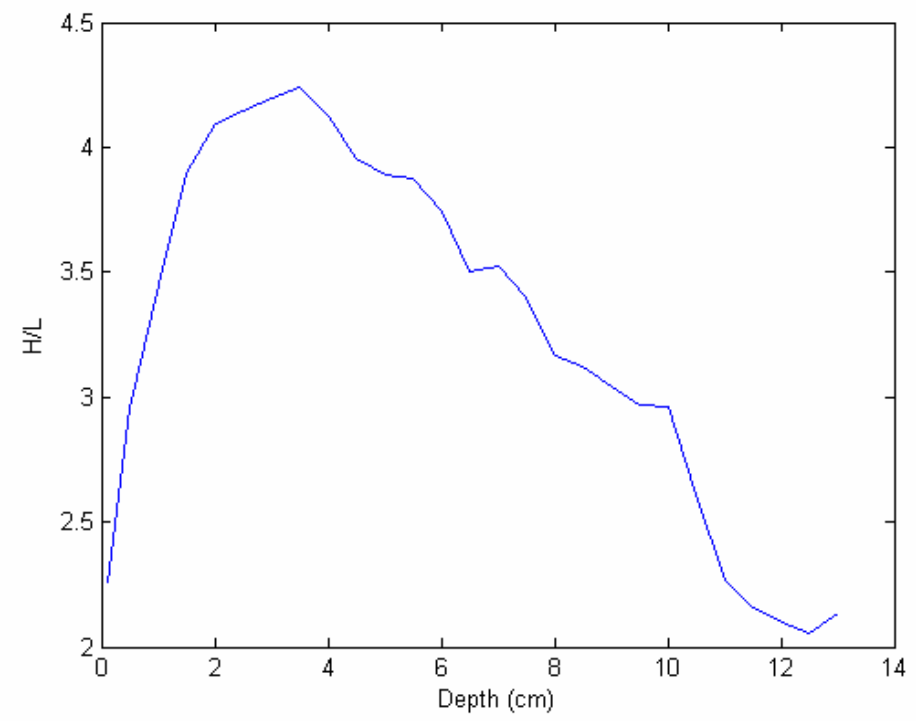

Figure 92: H/L (Tumor/healthy dose); Case 16 


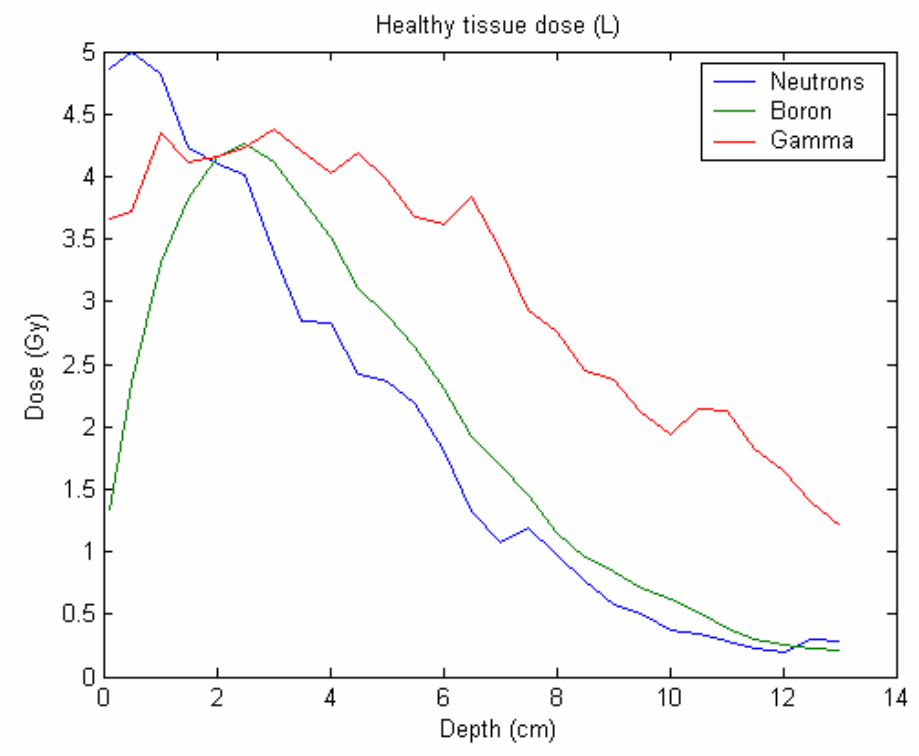

Figure 93: Components of the healthy tissue dose (L); Case 16

The maximum dose of about $51.8 \mathrm{~Gy}$ is reached at $2.5 \mathrm{~cm}$ depth in about 10 hours. The dose rate is $5.1 \mathrm{~Gy} /$ hour. The different relative contributions to the healthy tissue dose, shown in Figure 932, are balanced, similarly to Case Ten. This confirms that the alumina reflector is, indeed, responsible for the balanced dose contribution to the healthy tissue found in the reference design.

\subsection{Summary}

Table V summarizes selected parameters of the sixteen cases reported in Section 6. The most important parameter is the dose rate (Gy/hour); the higher it is the better. At the same time it is desirable to have large maximum dose, large $\mathrm{H} / \mathrm{L}$ dose ratio and large lethality range. Cases 8 and 11 are the most promising designs examined from the point of view of the dose rate: Case 11 offers the highest dose rate $(\mathrm{H})$ of $10.1 \mathrm{~Gy} /$ hour and can deliver the lethal dose of 20 Gy or higher to the tumor in the first $6 \mathrm{~cm}$ of brain tissue in 3.4 hours, while Case 8 offers a $\sim 14 \%$ larger total maximum dose at the expense of $\sim 10 \%$ lower peak dose rate. In both of these preferred designs the neutron dose component is significantly larger than the gamma dose component. 
Table V: Comparison of Selected Characteristics of the Cases Examined in Section 6

\begin{tabular}{|c|c|c|c|c|c|}
\hline Case \# & $\begin{array}{c}\text { Peak dose } \\
\text { rate (Gy/h) }\end{array}$ & $\begin{array}{c}\text { Maximum } \\
\text { dose (Gy) }\end{array}$ & $\begin{array}{c}\text { Treatment } \\
\text { time (hours)* }\end{array}$ & $\begin{array}{c}\text { Peak } \\
\text { H/L }\end{array}$ & $\begin{array}{c}\text { Lethality range } \\
\text { (cm) }\end{array}$ \\
\hline 1 & 5.7 & 36.0 & 6.3 & 3.3 & 0 to 6 \\
\hline 2 & 5.7 & 43.0 & 7.5 & 4.3 & 0 to 6.5 \\
\hline 3 & 3.4 & 22.2 & 6.5 & 3.4 & 1 to 4 \\
\hline 4 & 6.5 & 17.0 & 2.6 & 3.4 & N/A \\
\hline 5 & 8.1 & 40.7 & 5.0 & 4.0 & 0 to 6 \\
\hline 6 & 5.5 & 44.2 & 8.0 & 4.3 & 0 to 6 \\
\hline 7 & 5.9 & 46.0 & 7.8 & 4.2 & 0 to 6 \\
\hline 8 & 8.9 & 40.0 & 4.5 & 4.0 & 0 to 6 \\
\hline 9 & 4.0 & 36.4 & 9.0 & 3.2 & 0 to 6 \\
\hline 10 & 4.6 & 47.5 & 10.3 & 3.4 & 0 to 6.7 \\
\hline 11 & 10.1 & 34.7 & 3.4 & 4.0 & 0 to 6 \\
\hline 12 & N/A & N/A & N/A & N/A & N/A \\
\hline 13 & 4.5 & 44.5 & 9.9 & 3.4 & 0 to 6.5 \\
\hline 14 & 4.3 & 48.0 & 11.3 & 4.0 & 0 to 6.5 \\
\hline 15 & 8.1 & 37.9 & 4.7 & 4.0 & 0 to 6 \\
\hline 16 & 5.1 & 51.8 & 10.1 & 4.2 & 0 to 7 \\
\hline
\end{tabular}

* Treatment time is the time required to reach the healthy tissue dose constraint of $12.5 \mathrm{~Gy}$. Any tumor in the lethality range should be killed within this treatment time.

The largest maximum dose and the largest lethality range are offered by the designs of Case 16 followed by Case 14 and Case 10. In all of these cases the neutron and gamma contributions to the skin dose are equivalent.

The reason for the superior performance of the design of Case 11 over the reference BSA design (Case 1) can be understood with the help of Figure 95 to Figure 100. Figure 94 shows the ratio of the absorbed tumor dose to the maximum healthy tissue dose, or therapeutic gain, for tumors at different depths and for the skin as a function of the neutrons energy. Focusing on the $6 \mathrm{~cm}$ and $8 \mathrm{~cm}$ depths it is seen that the therapeutic gain increases monotonically up to about 10 $\mathrm{keV}$, and then drops fast so that by about $20 \mathrm{keV}$ the damage to the healthy tissue due to proton 
recoil reaction is about as big as the damage induced to the tumor and then becomes the biggest effect. Therefore we qualitatively see what was justified in [1] with a comprehensive study using mono-energetic beams to determine the most effective energy for the neutrons, the conclusions of which are that the optimal neutron energy is $7.94 \mathrm{keV}$, and the spectral components beyond 20 $\mathrm{keV}$ should be minimized.

Figure 95 shows the components of the dose delivered by $7.94 \mathrm{keV}$ mono-energetic neutron beam, peaking well inside the brain mass. On the other hand, a similar graph for a $20 \mathrm{keV}$ monoenergetic neutron beam, (in Figure 96), shows that the maximum dose by these neutrons is given to the skin, limiting the achievable therapeutic benefits.

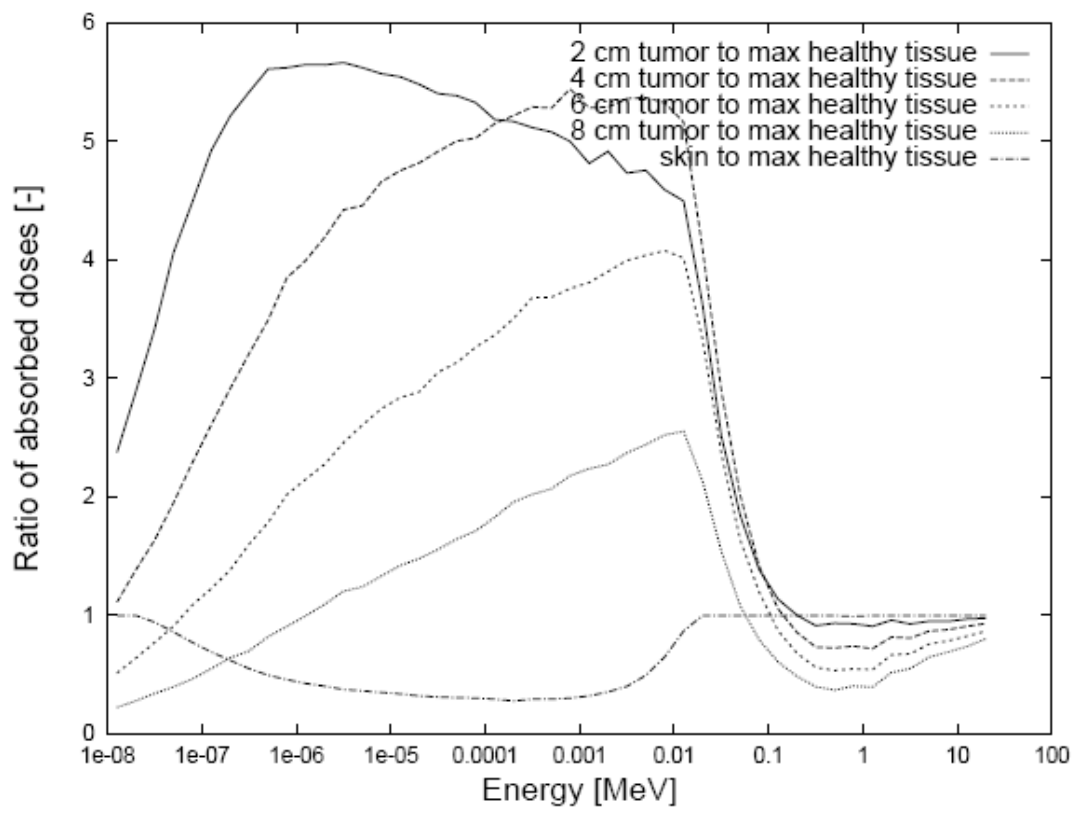

Figure 94: Ratios of absorbed doses versus neutron energy for a 12-cm-diameter mono-directional neutron beam (from [1]). 


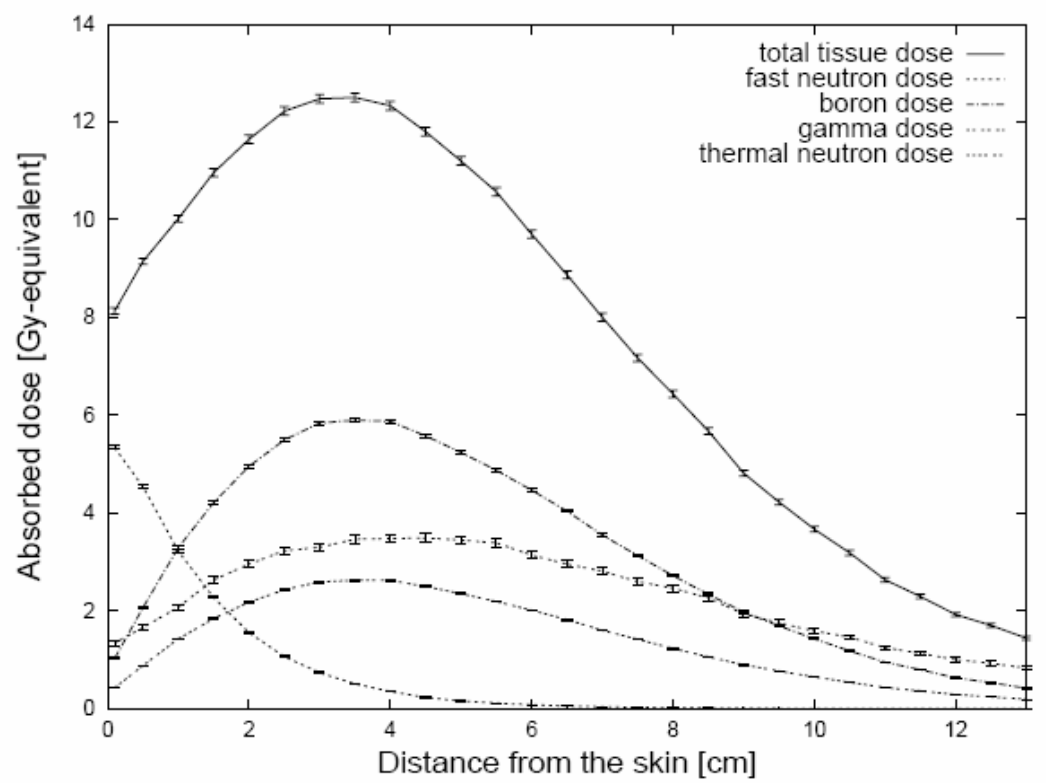

Figure 95: $7.94 \mathrm{keV}$ mono-energetic neutron beam (12 cm diameter): optimal energy of the neutrons based on mono-energetic studies (from [1]).

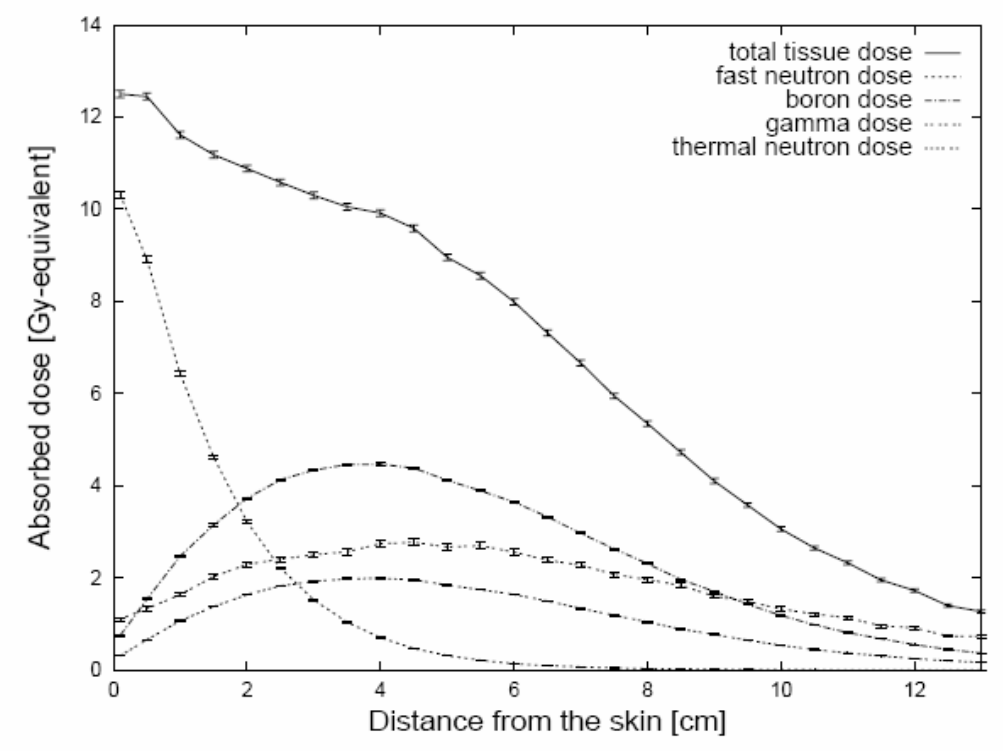

Figure 96: $20 \mathrm{keV}$ mono-energetic neutron beam (12 cm diameter): upper limit of the therapeutically beneficial neutrons. (from [1])

Figure 97 shows the spectrum of the neutrons exiting the BSA of Case 1 - i.e., the reference BSA; it is very similar to the spectrum of Verbeke's optimized design [1] shown in Figure 98. In fact the BSA design in [2] was taken from [1]. In both these designs the neutron energy peaks at less than $1 \mathrm{keV}$, making this spectrum too soft for the best possible treatment of deep-seated tumors. At the more desirable energy of about $10 \mathrm{keV}$, the neutron intensity is about half the 
peak value. On the other hand, the exit neutron spectrum in Case 11 (see Figure 99) peaks at the best possible energy of about $7 \mathrm{keV}$ while still decreasing rapidly before the un-desirable energy of $20 \mathrm{keV}$.

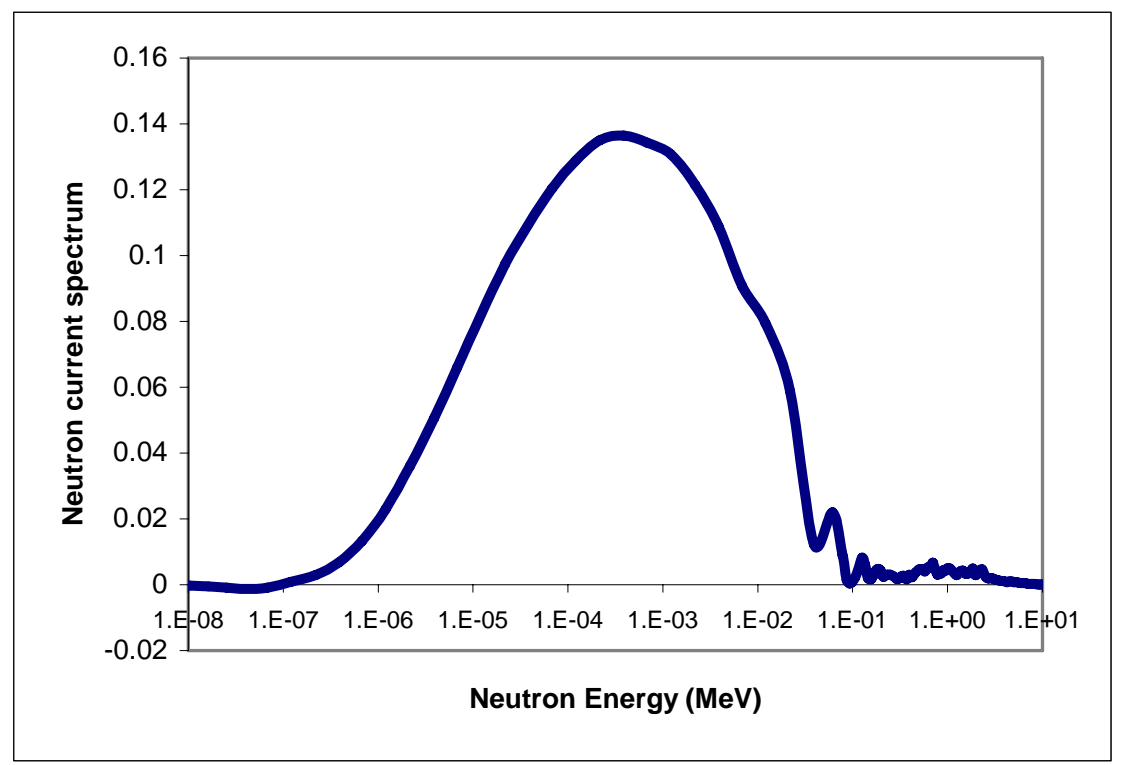

Figure 97: Exit spectrum of the neutrons for Case 1 (reference).

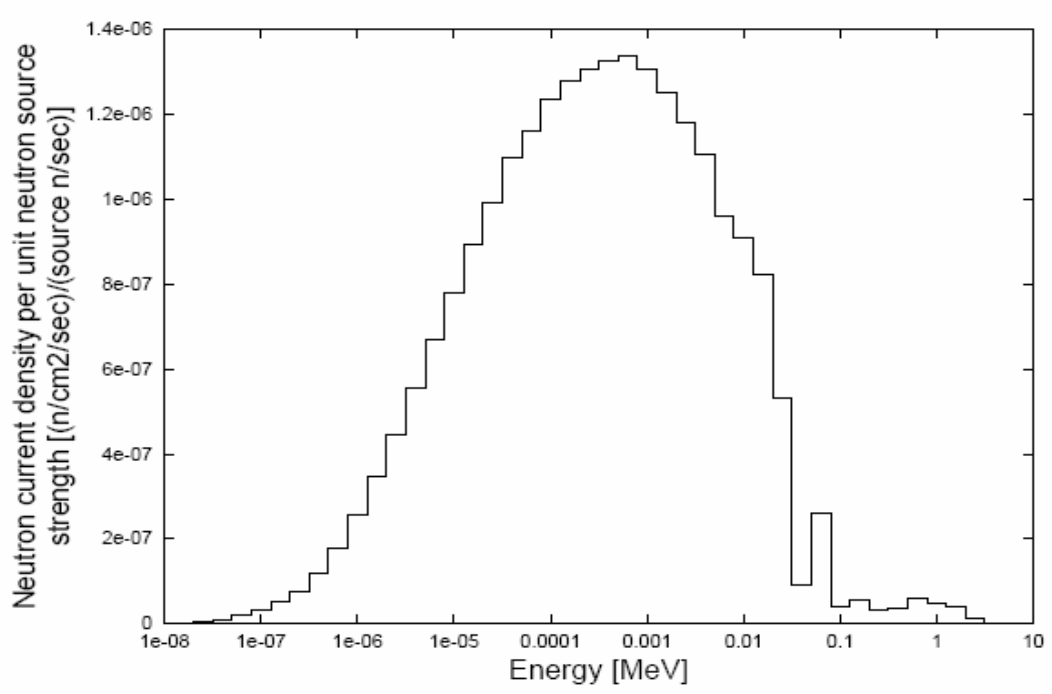

Figure 98: Exit spectrum of the neutrons for the optimized Verbeke case, (taken from [1]). 


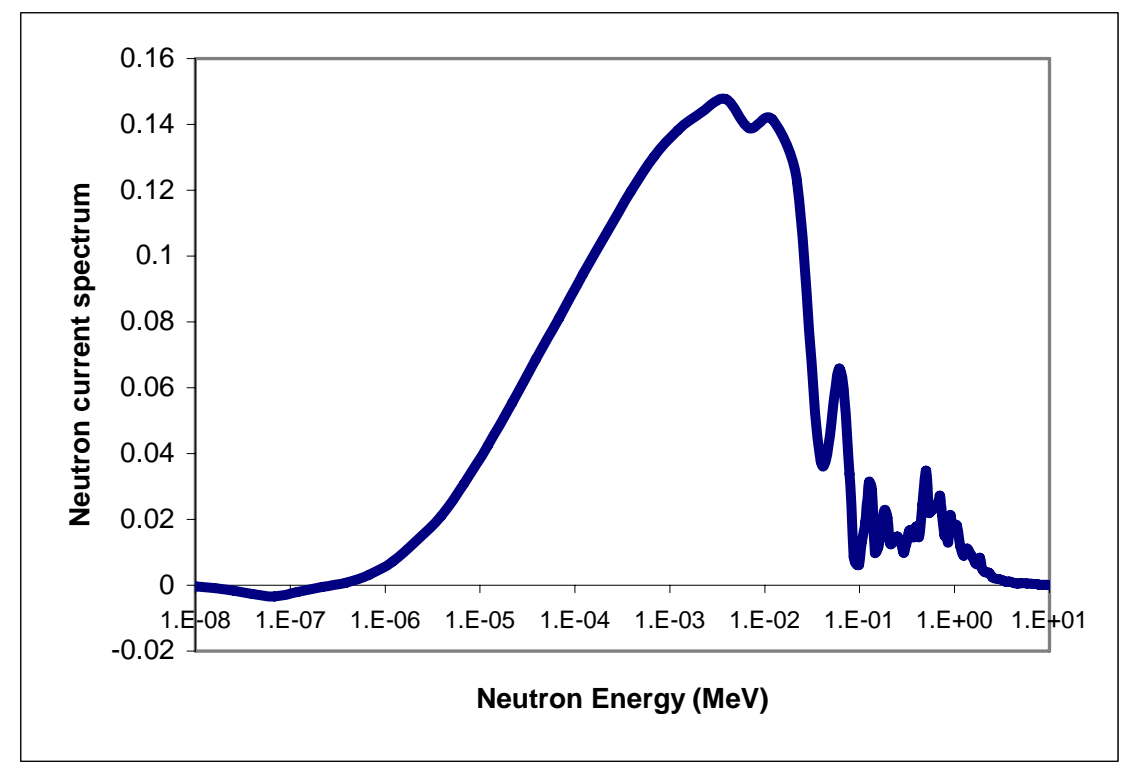

Figure 99: Exit spectrum of the neutrons for case eleven.

Figure 100 shows a comparison of the spectra of Cases 11 and 1. Case 11 spectrum is significantly harder but also have a relatively high tail of $\sim>50 \mathrm{KeV}$ neutrons; it is the preferred spectrum for treating deep seated tumors.

Figure 101 shows a similar comparison between Cases 11 and 16. The reduction in the fast current in Case 16 is responsible for a lower skin dose: this in turn translates into a higher healthy tissue dose and consequently a larger lethality range. As compared to Case 1, Case 16 has a slightly harder spectrum, which is beneficial. 


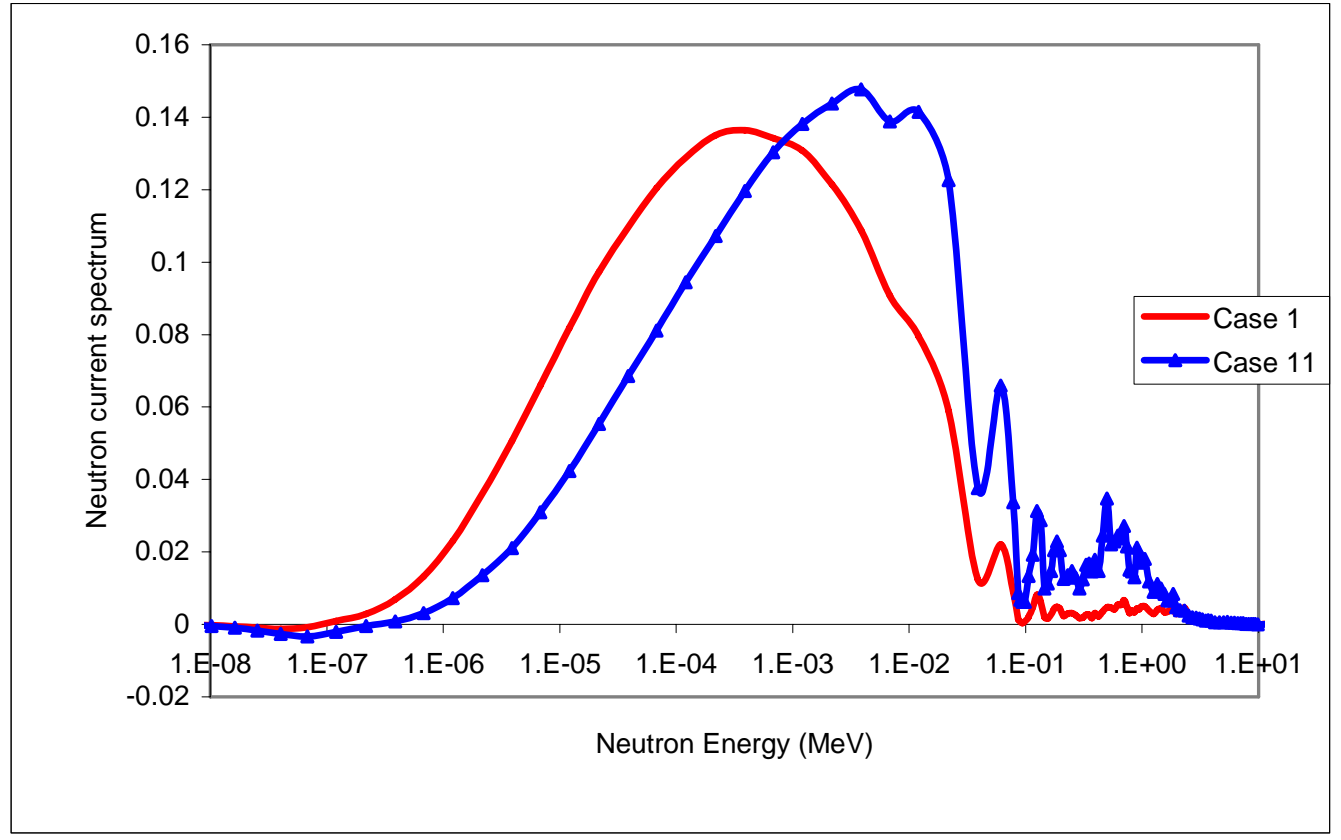

Figure 100: Exit spectrum of the neutrons for Case 1 compared to Case 11.

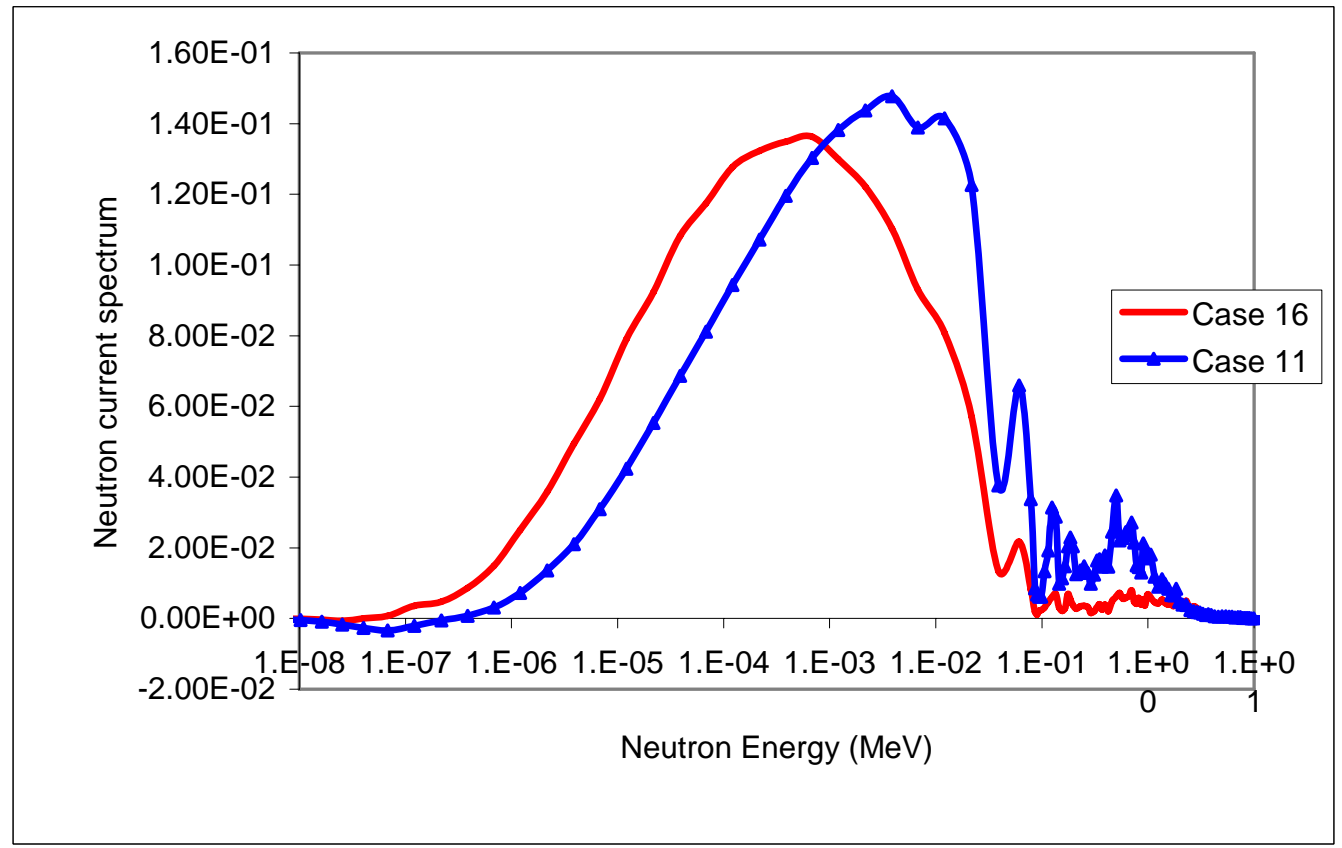

Figure 101: Exit spectrum of the neutrons for Case 16 compared to Case 11 


\section{DISCUSSION}

Even though the original goal of up to 1 hour treatment time using a single beam and $10^{12} \mathrm{D}-\mathrm{D}$ neutrons per second source has not been achieved, it appears possible to develop a practical BNCT facility based on the optimal system identified in this study by either

o Irradiating the patient in 3 to 4 one-hour sessions

o Irradiating the patient using 3 or 4 beams simultaneously

o Enhancing the capacity of the CNS to $\sim 5 \times 10^{12} \mathrm{D}-\mathrm{D} \mathrm{n} / \mathrm{s}$.

At the termination of this project we have been told by Dr. Ka Ngo Leung, the developer of the $\mathrm{CNS}$, that they now have designs that could deliver up to $10^{13} \mathrm{D}-\mathrm{D} \mathrm{n} / \mathrm{s}$. If this is proven practical, the BNCT facility designed in this project will be able to reach the project objective.

The SCM power level is estimated at $400 \mathrm{~W}$ when driven by a $10^{12} \mathrm{D}-\mathrm{D} \mathrm{n} / \mathrm{s}$ neutron source. This translates into consumption of only about $0.5 \%$ of the initially loaded ${ }^{235} \mathrm{U}$ atoms during 50 years of continuous operation. It thus appears that the SCM could operate continuously for the entire lifetime of the machine without refueling. Cooling the SCM does not pose a challenge; it may be accomplished passively; i.e., without resorting to forced circulation.

With $200 \mathrm{kV}$ extraction voltage, $1 \mathrm{~A}$ beam current and $50 \%$ efficiency in converting the grid power to beam power, the estimated power requirement of the $10^{12} \mathrm{D}-\mathrm{D} \mathrm{n} / \mathrm{sec} \mathrm{CNS}$ is estimated to be $400 \mathrm{~kW}$; this is 3 orders more than the fission power. Had one not used the SCM, the CNS power required to deliver a treatment neutron beam intensity comparable to that obtained with the SCM would be over $10 \mathrm{MW}$. In view of the fact that it is so much more energy (and cost) expensive to generate a D-D neutron than an excess fission neutron, it is suggested to explore the feasibility of licensing a small, inherently safe SCM featuring $k_{\text {eff }}$ that is larger than 0.98 ; possibly as large as 0.998 . Such a SCM will provide an order of magnitude higher neutron multiplication than that obtained from the SCM designed in this project.

The study focused on the use of uranium enriched to up to $20 \%$ for the SCM. Had higher enrichment been acceptable or had ${ }^{233} \mathrm{U}$ been used as the primary fissile isotope, the fraction of the fission neutrons that could leak out from the SCM per D-D driving neutron could have been 
increased, thus the treatment time could have been shortened without exceeding the $\mathrm{k}_{\mathrm{eff}}=0.98$ constraint.

\section{CONCLUSIONS}

The primary objective of this project was to assess the feasibility of using a small, safe and inexpensive subcritical fission assembly to multiply the treatment neutron beam intensity attainable from a compact D-D fusion neutron source delivering $10^{12} \mathrm{n} / \mathrm{s}$ by a factor of $\geq 30$ so as to provide a compact BNCT facility that could treat deep-seated brain tumors in one hour. The study concludes that it is not quite feasible to achieve this objective. Nevertheless, it appears feasible to develop a practical BNCT facility based on the optimal system identified in this study by either

o Irradiating the patient in 3 to 4 one-hour sessions

o Irradiating the patient using 3 or 4 beams simultaneously

o Enhancing the capacity of the CNS to $\sim 5 \times 10^{12} \mathrm{D}-\mathrm{D} \mathrm{n} / \mathrm{s}$

o Increasing the permissible SCM maximum $\mathrm{k}_{\text {eff }}$ to 0.995

The study identified the optimal design of a SCM made of up to $20 \%$ enriched uranium fuel that is clad with aluminum that maximizes the current of neutrons that leak out from the SCM in the direction of the patient, without exceeding the maximum permissible $\mathrm{k}_{\text {eff }}$ of 0.98 . This optimal design is made of $20 \%$ enriched uranium metal arranged as follows: two cylindrical fuel shells surrounds the CNS coaxially and four circular fuel elements are locate in-between the CNS and the BSA. The coaxial cylinders are $21 \mathrm{~cm}$ long - as is the CNS, and $0.06 \mathrm{~cm}$ thick and are clad with $0.05 \mathrm{~cm}$ thick aluminum on both sides. Water fills the $0.84 \mathrm{~cm}$ gap between them. The innermost cylinder has a radius of $13.5 \mathrm{~cm}$. The disc-shaped fuel plates are, similarly, $0.06 \mathrm{~cm}$ thick, clad with $0.05 \mathrm{~cm}$ thick aluminum and have $0.84 \mathrm{~cm}$ thick water gaps in-between. The entire SCM is encased in aluminum structure that provides a physical barrier between the CSN and the SCM. A $0.84 \mathrm{~cm}$ thick water layer separates the outermost fuel element and the encasement. The total amount of enriched uranium in the SCM is $8.5 \mathrm{~kg}$ and its cost (not including fabrication) is estimated to be less than $\$ 60,000$. The SCM power level is estimated at $400 \mathrm{~W}$ when driven by a $10^{12} \mathrm{D}-\mathrm{D} \mathrm{n} / \mathrm{s}$ neutron source. This translates into consumption of only 
about $0.5 \%$ of the initially loaded ${ }^{235} \mathrm{U}$ atoms during 50 years of continuous operation. It implies that the SCM could operate continuously for the entire lifetime of the machine without refueling. Cooling the SCM does not pose a challenge; it may be accomplished passively; i.e., without resorting to forced circulation.

Two optimal BSA designs were identified; one for maximizing the dose rate to a deep seated tumor and the other for maximizing the total dose that can be delivered to a deep seated tumor. The former offers the minimum treatment time whereas the latter offers a larger lethality range. The former features a harder neutron spectrum and relatively high neutron dose component to the skin while in the latter the neutron, gamma-ray and boron dose components in the skin are comparable. The maximum dose rate that can be delivered by the former is $10.1 \mathrm{~Gy} /$ hour and the maximum dose that can be delivered by the latter is $51.8 \mathrm{~Gy}$.

The BSA design that maximizes the dose rate is $51.1 \mathrm{~cm}$ long, with a truncated conical shape with the large base towards the SCM and the small base towards the patient. The angle of the cone is 14.9 degrees, the large base towards the SCM has a radius of $23.3 \mathrm{~cm}$ while the small base towards the patient has a radius of $9.74 \mathrm{~cm}$. The BSA is made of the following segments, starting from the SCM with the thickness indicated in $\mathrm{cm}: 3.5 \mathrm{~cm}$ lead, $6 \mathrm{~cm}{ }^{7} \mathrm{LiF}, 1.5 \mathrm{~cm}$ lead, $17 \mathrm{~cm}{ }^{7} \mathrm{LiF}, 4 \mathrm{~cm}$ fluental, $1 \mathrm{~cm}$ lead, $8 \mathrm{~cm}$ fluental, $1 \mathrm{~cm}$ lead, $8 \mathrm{~cm}$ fluental, $0.9 \mathrm{~cm}$ lead and 0.1 $\mathrm{cm}^{6} \mathrm{LiF}$. The cone is surrounded on the side by a $1 \mathrm{~mm}$ thick ${ }^{6} \mathrm{LiF}$ in the last $19 \mathrm{~cm}$ towards the patient. The reflector is made of lead, and fills the space between the side of the BSA cone and a coaxial cylinder $77 \mathrm{~cm}$ in radius. The sides of the collimator are shielded by a lead collar $2.1 \mathrm{~cm}$ thick, continuing the conical shape of the BSA.

The BSA design that maximizes the total dose is $53.1 \mathrm{~cm}$ long, with a truncated conical shape with the large base towards the SCM and the small base towards the patient. The angle of the cone is 14.9 degrees, the large base towards the SCM has a radius of $23.3 \mathrm{~cm}$ while the small base towards the patient has a radius of $9.18 \mathrm{~cm}$. The BSA is made of the following segments, starting from the SCM with the thickness indicated in $\mathrm{cm}: 3.5 \mathrm{~cm}$ lead, $27.4 \mathrm{~cm}{ }^{7} \mathrm{LiF}, 20 \mathrm{~cm}$ fluental, $0.1 \mathrm{~cm}{ }^{6} \mathrm{LiF}$ and $2.1 \mathrm{~cm}$ lead. The cone is surrounded on the side by a $1 \mathrm{~mm}$ thick ${ }^{6} \mathrm{LiF}$ in the last $21 \mathrm{~cm}$ towards the patient. The reflector is made of $\mathrm{Al}_{2} \mathrm{O}_{3}$, filling the space between 
the side of the BSA cone and a coaxial cylinder $35 \mathrm{~cm}$ in radius; lead surrounds the alumina reflector with a coaxial cylinder $77 \mathrm{~cm}$ in external radius. The sides of the collimator are shielded by a lead collar $2.1 \mathrm{~cm}$ thick, continuing the conical shape of the BSA.

\section{REFERENCES}

1. J. M. Verbeke, J. Vujic, and K. N. Leung, "Neutron Beam Optimization for Boron Neutron Capture Therapy Using the D-D and D-T High-Energy Neutron Sources," Nuclear Technology, 129, 257 (2000).

2. C.M. Goff, "Design of a BNCT Medical Facility for Use with a D-D Fusion-based Compact Neutron Generator", M.S. thesis, University of California, Berkeley, August 2005.

3. H. Koivunoro, T-P Lou, J. Reijonen and K-N Leung, "A Compact Neutron Generator Based on D-D or D-T Fusion Reaction for BNCT," Proceedings of the10th International Congress on Neutron Capture Therapy for Cancer, Essen, Germany, 8 - 13 September 2002.

4. F.M. Bacon, D.F. Cowgill, C.E. Hickox, R.J. Walko, S.R. Subia, Jr., and A.A. Riedel, "High Heat Flux Target for Intense Neutron Source", Rev. Sci. Instrum. 55 (1), (January 1984).

5. Oak Ridge National Laboratory "RSICC Computer Code Collection MCNP4C: Monte Carlo N-Particle Transport Code System", Radiation Safety Information Computational Center, 3-150 p.p., Jul 2000.

6. M.D. DeHart, 2005. TRITON: A Two Dimensional Depletion Sequence for Characterization of Spent Nuclear Fuel, Nuclear science and technology division (94), Oak Ridge National Laboratory.

7. M.D. DeHart, 2005. NEWT: a New Transport Algorithm for Two-Dimensional Discrete Ordinates Analysis in Non-Orthogonal Geometries", Nuclear science and technology division (94), Oak Ridge National Laboratory.

8. W.S. Snyder, M.R. Ford, and G.G. Ford, "Estimates of Specific Absorbed Fractions for Photon Sources Uniformly Distributed in Various Organs of a Heterogeneous Phantom," MIRD Pamphlet 5 Revised, Society of Nuclear Medicine, New York, 1978.

9. "Photon, Electron, Proton and Neutron Interaction Data for Body Tissues," ICRU 46, International Commission on Radiation Units and Measurement, 1992. 
10. R.S. Caswell, J.J Coyne, and M.L. Randolph "KERMA Factors of Elements and Compounds for Neutron Energies Below $30 \mathrm{MeV}$," International Journal of Applied Radiation Isotopes, Vol. 33, 1227-1262, 1982.

11. A.D. Chanana "Boron Neutron Capture Therapy of Glioblastoma Multiforme at the Brookhaven Medical Research Reactor, A Phase I/II Study," FDA IND 43317, Protocol 4, Brookhaven National Laboratory, 1996.

12. Y. Karni, D. Regev, E. Greenspan, S. Goluoglu, L.M. Petrie and C.M. Hopper, "The SMORES Capability for Minimum Critical Mass Determination," Proceedings of the International Conference on Nuclear Criticality Safety (ICNC2003), Tokai-Mura, Japan, October 20-24, 2003.

13. E. Greenspan and Y. Karni, BNCT Beam Quality Maximization, $8^{\text {th }}$ International Symposium on Neutron Capture Therapy for Cancer, La Jolla, CA, Sept. 13-18, 1998.

14. E. Greenspan and Y. Karni, The "SWAN" Approach to Neutron Beam Optimization for BNCT and Other Applications, International Conf. on the Physics of Nucl. Sci. \& Technol., Long Island, NY, Oct. 5-8, 1998.

15. Y. Karni, E. Greenspan and J. Vujic, Accelerator Driven Fission Neutron Source for BNCT, ANS Embedded Topical Mtg. On Nuclear Applications of Accelerator Technology, Albuquerque, NM, Nov. 16-20, 1997. 


\section{APPENDIX A - Reflector Materials}

\section{A.1 INTRODUCTION}

The objective of this study was to rank different materials by their suitability to serve as a reflector for a small critical assembly. The critical assembly examined is a minimum mass spherical core made of a mixture of UZrH1.6 with $20 \%$ enriched $\mathrm{U}$. The calculations were done using MCNP. Three parameters where compared for each of the reflector materials: the multiplication eigenvalue $\left(\mathrm{k}_{\mathrm{eff}}\right)$, the total current escaping from the reflector and the fast neutron current (neutron energy $>1 \mathrm{keV}$ ) escaping from the reflector. Two reflector thicknesses where examined: $20 \mathrm{~cm}$ and $120 \mathrm{~cm}$. $\mathrm{BeO}$ was selected as the preferred reflector material for the BNCT facility as in minimizes the amount and cost of the required uranium and contributes to the safety of the facility: the replacement of $\mathrm{BeO}$ or part thereof with any other material, possibly as a result of an accident, will result in a negative reactivity effect.

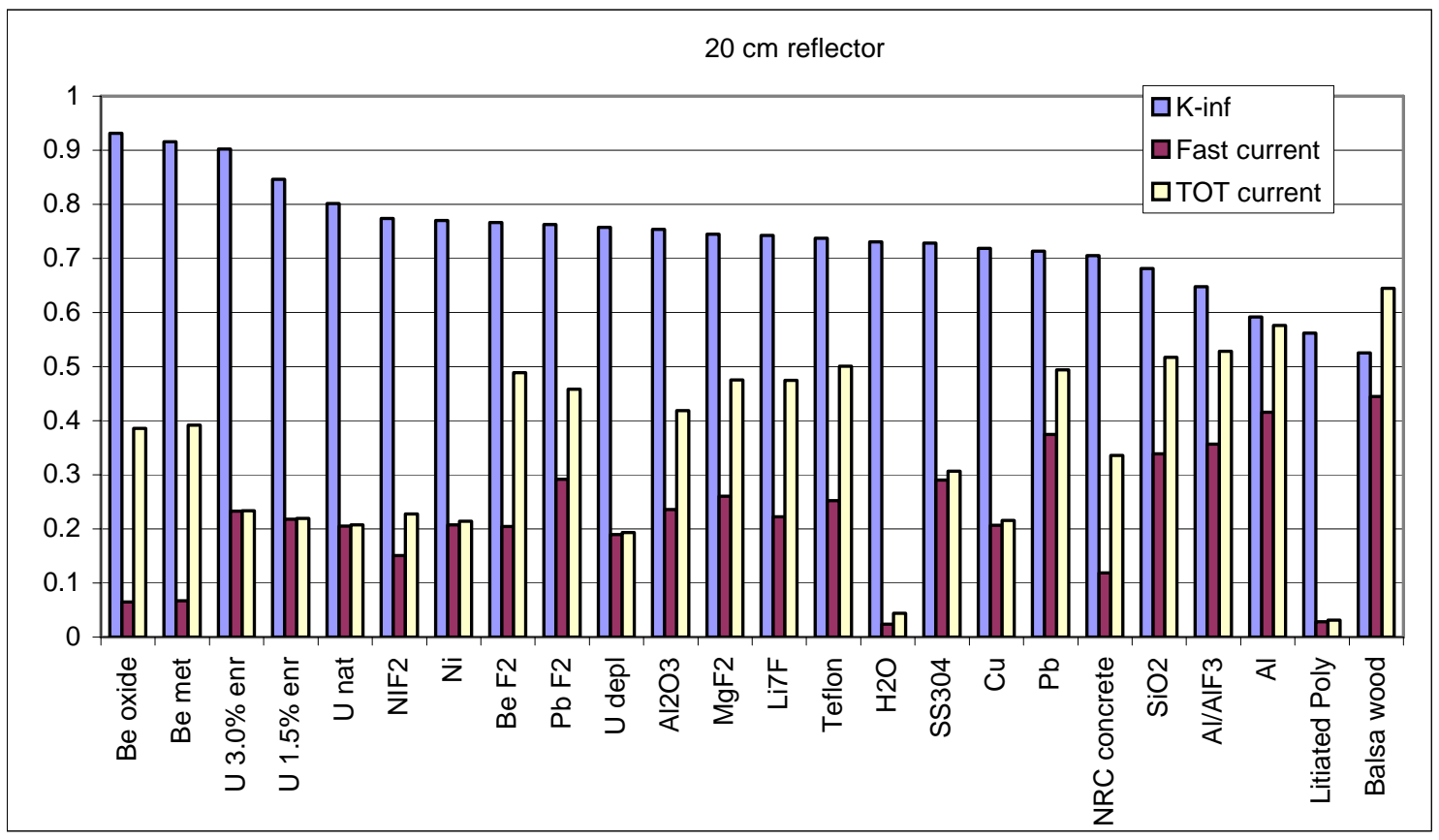

Figure 102: Comparison of the effectiveness of various materials as reflectors $20 \mathrm{~cm}$ thick. 


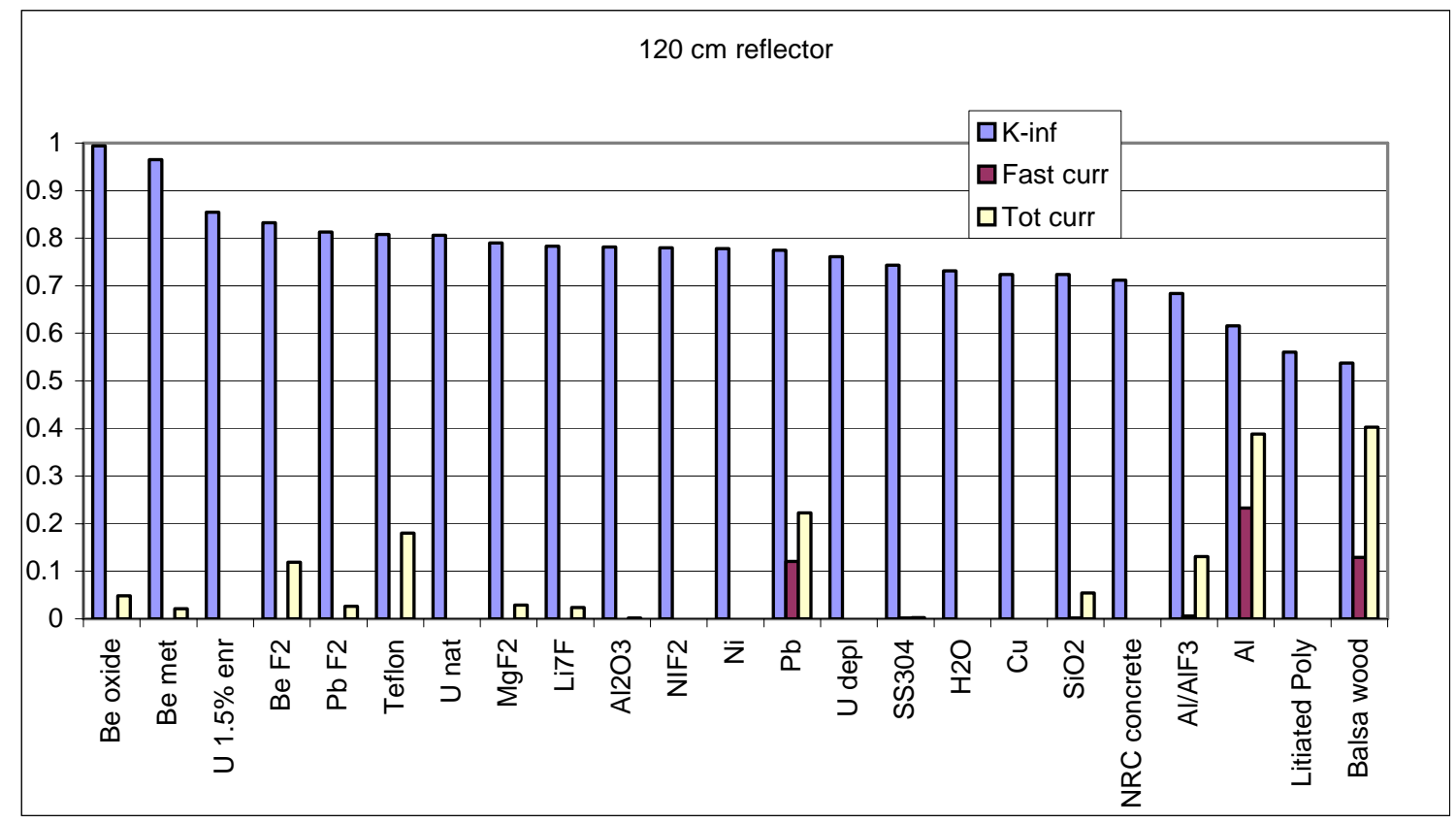

Figure 103: Comparison of the effectiveness of various materials as reflectors $120 \mathrm{~cm}$ thick. 